Supporting Information

for

\title{
PCET Enables Tandem Radical Relay for Asymmetric Copper-Catalyzed Phosphinoylcyanation of Styrenes
}

\author{
Guoyu Zhang, ${ }^{a}$ Liang Fu, ${ }^{\mathrm{b}}$ Pinhong Chen, ${ }^{\mathrm{b}}$ Jianping Zou, ${ }^{\mathrm{a}}$ and Guosheng Liu*b,c \\ ${ }^{a}$ Key Laboratory of Organic Synthesis of Jiangsu Province, College of Chemistry and Chemical \\ Engineering, Soochow University, Jiangsu 215123, China. ${ }^{b}$ State Key Laboratory of \\ Organometallic Chemistry, and ${ }^{c}$ Shanghai Hongkong Joint Laboratory in Chemical Synthesis, \\ Center for Excellence in Molecular Synthesis, Shanghai Institute of Organic Chemistry, Chinese \\ Academy of Sciences, 345 Lingling Road, Shanghai 200032, China \\ Email: gliu@mail.sioc.ac.cn;jpzou@suda.edu.cn
}

\section{Table of Contents}

$\begin{array}{lll}\text { 1. General S2 } & \text { S2 }\end{array}$

2. General Experimental Procedure $\quad$ S2

3. Scaled up Reaction S3

4. Synthetic Applications $\quad$ S4

$\begin{array}{ll}\text { 5. Mechanistic Studies } & \text { S7 }\end{array}$

6. Characterization of the Products $\quad$ S16

$\begin{array}{ll}\text { 7. X-Ray Data (3a) } & \text { S35 }\end{array}$ 


\section{General}

All commercially available compounds were purchased from Aldrich, Alfa Aesar or Adamas. NMR spectra were recorded on Varian Inova 400 and Agilent 400 (400 MHz for ${ }^{1} \mathrm{H}$; $160 \mathrm{MHz}$ for ${ }^{31} \mathrm{P} ; 100 \mathrm{MHz}$ for $\left.{ }^{13} \mathrm{C}\right)$ spectrometer. The chemical shifts $(\delta)$ are given in parts per million relative to $\mathrm{CDCl}_{3}(7.26 \mathrm{ppm}$ for $1 \mathrm{H})$ or TMS $(0 \mathrm{ppm}$ for $1 \mathrm{H})$ and $\mathrm{CDCl}_{3}\left(77.0 \mathrm{ppm}\right.$ for $\left.{ }^{13} \mathrm{C}\right)$. High performance liquid chromatography was performed on Waters 2487-600E, Waters ACQUITY UPC2, and Ultimate 3000 Series HPLC, using IC-3, IG, IA and IF-3 chiral column eluted with a mixture of hexane and isopropyl alcohol. Optical rotation was measured on a Rudolph-Autopol I. Flash column chromatography was performed on silica gel (particle size 200-300 mesh, purchased from Canada) and eluted with EA/PE. Solvent was purified according to the procedure from the book named -Purification of Laboratory Chemicals.

\section{General Experimental Procedure}
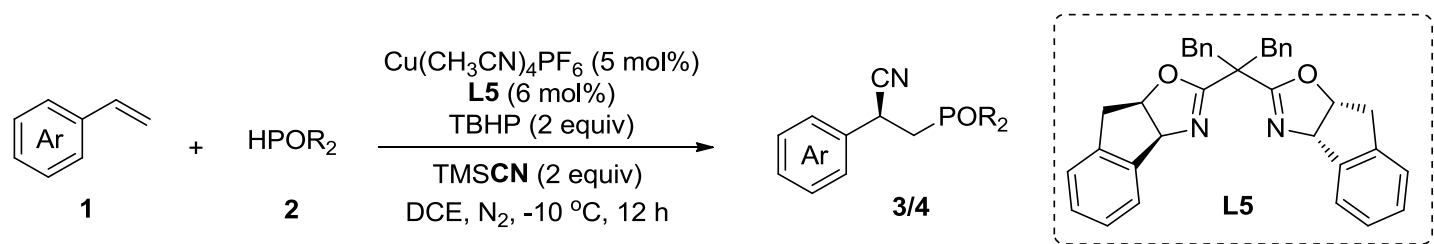

To a $10 \mathrm{~mL}$ sealed tube, ligand $\mathbf{L 5}(6.2 \mathrm{mg}, 0.012 \mathrm{mmol}, 6 \mathrm{~mol} \%)$ and $\mathrm{Cu}\left(\mathrm{CH}_{3} \mathrm{CN}\right)_{4} \mathrm{PF}_{6}(3.7 \mathrm{mg}, 0.010 \mathrm{mmol}, 5 \mathrm{~mol} \%)$ were dissolved in anhydrous and degassed DCE $(1.0 \mathrm{~mL})$ under $\mathrm{N}_{2}$. The colorless solution was stirred at $-10{ }^{0} \mathrm{C}$ for 15 min, then substrate 1 (0.2 mmol, 1.0 equiv.), HPOR 2 (0.4 mmol, 2.0 equiv.), TMSCN (52 uL, 0.4 mmol, 2.0 equiv.) and ${ }^{t} \mathrm{BuOOH}(72.0 \mu \mathrm{L}, 0.4 \mathrm{mmol}, 2.0$ equiv., $5.5 \mathrm{~mol} / \mathrm{L}$ in decane) were sequentially added at $-10{ }^{\circ} \mathrm{C}$. Notably, when ${ }^{t} \mathrm{BuOOH}$ was added, the reaction mixture turned purple. The reaction was still stirred at $-10{ }^{\circ} \mathrm{C}$ for $12 \mathrm{~h}$ before the reaction mixture turned colorless. After reaction completion, 5\% $\mathrm{Na}_{2} \mathrm{~S}_{2} \mathrm{O}_{3}$ cooled aqueous solution $(2 \mathrm{~mL})$ were added, and the reaction mixture was stirred for 30 mins. Then the aqueous phase was extracted with DCM $(3 \times 10 \mathrm{~mL})$. The combined organic phase was washed with brine, dried over anhydrous $\mathrm{Na}_{2} \mathrm{SO}_{4}$ and 
concentrated in vacuum. The residue was purified by flash column chromatography on silica gel with a gradient of petroleum ether/acetone to afford the desired products 3-4 as shown in Table 2 and 3.

Cautious! Owing to the involvement of HCN in the catalytic system, all the procedure were performed in fume hood in good condition, and the reactions were conducted in the sealed bottle. After completed the reaction, $5 \% \mathrm{Na}_{2} \mathrm{~S}_{2} \mathrm{O}_{3}$ cooled aqueous solution was added to quench the excess amount of HCN to deliver low toxic NaSCN. Finally, the aqueous phase should be treated carefully by aqueous $\mathrm{KMnO}_{4} \mathrm{Or} \mathrm{NaOCl}$ solution.

\section{Scaled up Reaction}

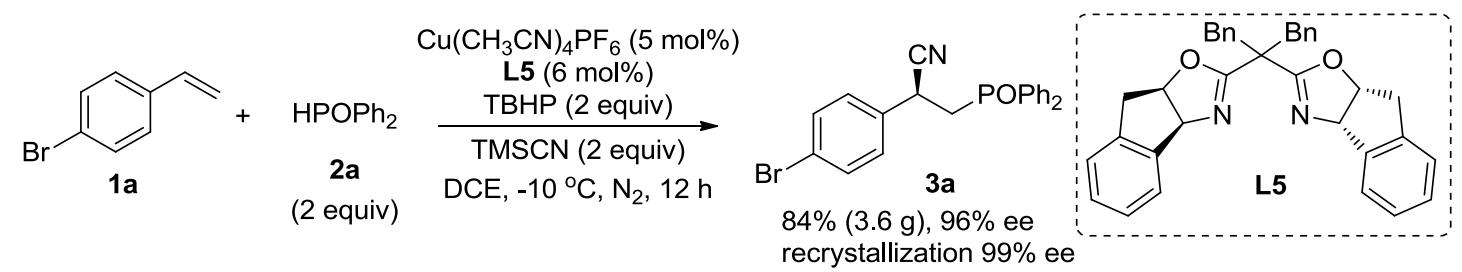

To a $250 \mathrm{~mL}$ sealed tube, ligand $\mathbf{L 5}(310 \mathrm{mg}, 0.5 \mathrm{mmol}, 6 \mathrm{~mol} \%)$ and $\mathrm{Cu}\left(\mathrm{CH}_{3} \mathrm{CN}\right)_{4} \mathrm{PF}_{6}(185 \mathrm{mg}, 0.5 \mathrm{mmol}, 5 \mathrm{~mol} \%)$ were dissolved in anhydrous and degassed DCE $(95 \mathrm{~mL})$ under $\mathrm{N}_{2}$, the mixture was stirred at $-10{ }^{0} \mathrm{C}$ for $15 \mathrm{~min}$ (the colorless solution A). At the same time, to a $10 \mathrm{~mL}$ sealed tube, TMSCN (2.6 mL, 20 mmol, 2.0 equiv.) and TBHP (3.6 mL, $20 \mathrm{mmol}, 2.0$ equiv.) were dissolved in anhydrous and degassed DCE $(5 \mathrm{~mL})$ under $\mathrm{N}_{2}$, the mixture was stirred at $-10{ }^{0} \mathrm{C}$ for $15 \mathrm{~min}$ (the colorless solution B). Then the substrate $\mathbf{1 a}(1.81 \mathrm{~g}, 10 \mathrm{mmol}, 1.0$ equiv.), $\mathrm{HPOPh}_{2}$ 2a (4.04g, $20 \mathrm{mmol}, 2.0$ equiv.) and the solution B were sequentially added into the solution $\mathrm{A}$ at $-10{ }^{\circ} \mathrm{C}$ and the reaction turned purple. After that, the reaction was still stirred at $-10{ }^{\circ} \mathrm{C}$ for $12 \mathrm{~h}$ before the reaction turned colorless. After reaction completion, 5\% $\mathrm{Na}_{2} \mathrm{~S}_{2} \mathrm{O}_{3}$ cooled aqueous solution $(20 \mathrm{~mL})$ were added, and the reaction mixture was stirred for 30 mins. Then the aqueous phase was extracted with DCM $(3 \times 100 \mathrm{~mL})$. The combined organic phase was washed with brine, dried over anhydrous $\mathrm{Na}_{2} \mathrm{SO}_{4}$ and concentrated in vacuum, and the residue was purified by flash 
column chromatography on silica gel with a gradient of PE/ Ac (4:1) to afford the product $3 \mathbf{a}$ as a white solid (3.6 g, $84 \%$ yield, $96 \%$ ee). The enantioselectivity of the product 3a could be increased to $99 \%$ after recrystallization.

Cautious! Owing to the involvement of HCN in the catalytic system, all the procedure were performed in fume hood in good condition, and the reactions were conducted in the sealed bottle. After completed the reaction, $5 \% \mathrm{Na}_{2} \mathrm{~S}_{2} \mathrm{O}_{3}$ cooled aqueous solution was added to quench the excess amount of HCN to deliver low toxic NaSCN. Finally, the aqueous phase should be treated carefully by aqueous $\mathrm{KMnO}_{4}$ or $\mathrm{NaOCl}$ solution.
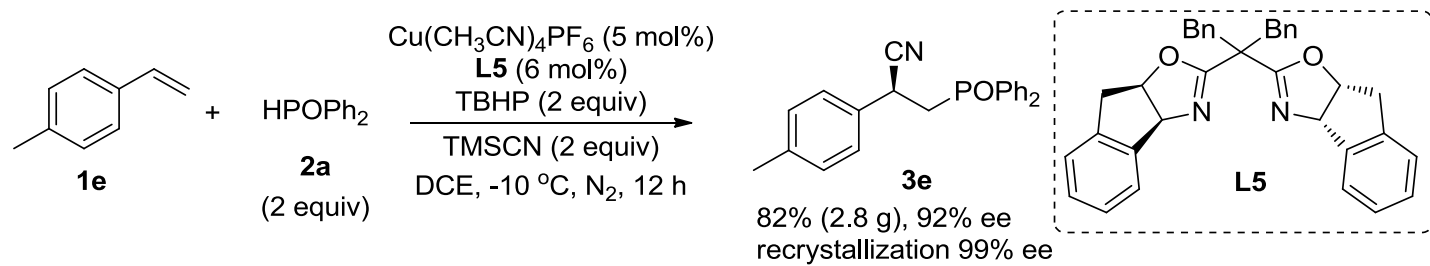

According to the same procedure, the reaction of 4-methylstyrene 1e (1.18 g, 10.0 mmol) gave $3 \mathbf{e}$ ( $2.8 \mathrm{~g}, 82 \%$ yield, $92 \%$ ee) as a white solid, and the enantioselectivity of the product $3 \mathbf{e}$ could be increased to $99 \%$ after recrystallization.

\section{Synthetic Applications}

\subsection{Reduction of the Products}
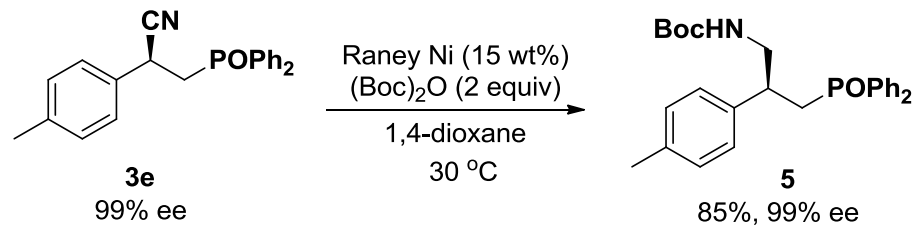

To a $4 \mathrm{~mL}$ glass vessel, the compound $3 \mathrm{e}$ (34.5 mg, $0.1 \mathrm{mmol}, 1.0$ equiv., $99 \%$ ee), Raney $\mathrm{Ni}(3.2 \mathrm{mg}, 15 \mathrm{wt} \%)$ and $\mathrm{Boc}_{2} \mathrm{O}$ (69.8 mg, $0.32 \mathrm{mmol}, 2.0$ equiv.) were dissolved in dioxane $(1 \mathrm{~mL})$. The glass vessel was placed in the autoclave, which was exchanged with $\mathrm{H}_{2}$ for three times and kept at 30 bar $\mathrm{H}_{2}$. The reaction was stirred at $30{ }^{\circ} \mathrm{C}$ for $24 \mathrm{~h}$. After that, the mixture was filtered through a pad of celite with EtOAc. 
The filtrate was evaporated under reduced pressure and the residue was purified by flash column chromatography on silica gel to afford $\mathbf{5}$ as a light white solid (38.2 $\mathrm{mg}$, $85 \%$ yield, $99 \%$ ee), mp. $115{ }^{\circ} \mathrm{C}-116{ }^{\circ} \mathrm{C} .{ }^{1} \mathrm{H} \mathrm{NMR}\left(400 \mathrm{MHz}, \mathrm{CDCl}_{3}\right) \delta 7.65-7.76$ $(\mathrm{m}, 2 \mathrm{H}), 7.65-7.52(\mathrm{~m}, 2 \mathrm{H}), 7.51-7.37(\mathrm{~m}, 4 \mathrm{H}), 7.36-7.27(\mathrm{~m}, 2 \mathrm{H}), 7.08-6.80$ $(\mathrm{m}, 4 \mathrm{H}), 5.80(\mathrm{~s}, 1 \mathrm{H}), 3.59-3.31(\mathrm{~m}, 2 \mathrm{H}), 3.27-3.17(\mathrm{~m}, 1 \mathrm{H}), 2.80-2.67(\mathrm{~m}, 1 \mathrm{H})$, $2.65-2.57(\mathrm{~m}, 1 \mathrm{H}), 2.29(\mathrm{~s}, 3 \mathrm{H}), 1.37(\mathrm{~s}, 9 \mathrm{H}) .{ }^{13} \mathrm{C} \mathrm{NMR}\left(100 \mathrm{MHz}, \mathrm{CDCl}_{3}\right) \delta 155.9$, $139.0(\mathrm{~d}, J=8.1 \mathrm{~Hz}), 136.2,133.5(\mathrm{~d}, J=99.4 \mathrm{~Hz}), 133.4$ (d, $J=99.4 \mathrm{~Hz}), 131.6$ (d, $J$ = 2.7 Hz), $131.2(\mathrm{~d}, J=2.4 \mathrm{~Hz}), 130.7(\mathrm{~d}, J=9.2 \mathrm{~Hz}), 130.2(\mathrm{~d}, J=9.3 \mathrm{~Hz}), 129.0$, $128.4(\mathrm{~d}, J=11.9 \mathrm{~Hz}), 128.2(\mathrm{~d}, J=11.9 \mathrm{~Hz}), 127.1,78.6,46.7(\mathrm{~d}, J=7.5 \mathrm{~Hz}), 39.8$ $(\mathrm{d}, J=3.5 \mathrm{~Hz}), 34.6(\mathrm{~d}, J=68.2 \mathrm{~Hz}), 28.2,20.7 .{ }^{31} \mathrm{P} \mathrm{NMR}\left(162 \mathrm{MHz}, \mathrm{CDCl}_{3}\right) \delta 32.6$. HRMS: m/z (ESI) calculated $[\mathrm{M}+\mathrm{H}]^{+}$450.2193, measured: 450.2194.

${ }^{[\alpha]} \mathrm{D}^{26.7} 5.8876\left(\mathrm{c} 0.25, \mathrm{CHCl}_{3}\right)$. HPLC (IC-3, 0.46*15 cm, $3 \mu \mathrm{m}$, hexane/ isopropanol $=6 / 4$, flow $0.7 \mathrm{~mL} / \mathrm{min}$, detection at $214 \mathrm{~nm}$ ) retention time $=9.893 \mathrm{~min}$ (minor) and $7.972 \mathrm{~min}$ (major).

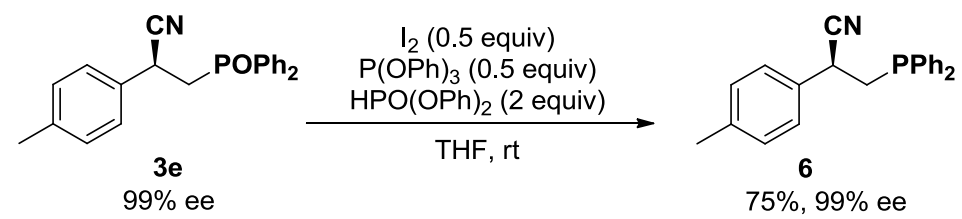

To a $10 \mathrm{~mL}$ sealed tube, $3 \mathrm{e}(34.5 \mathrm{mg}, 0.1 \mathrm{mmol}), \mathrm{P}\left(\mathrm{OPh}_{3}\right)(15.6 \mathrm{mg}, 0.05 \mathrm{mmol}$, 0.5 equiv. $)$ and $\mathrm{HPO}(\mathrm{OPh})_{2}(46.8 \mathrm{mg}, 0.2 \mathrm{mmol}, 2.0$ equiv. $)$ were dissolved in anhydrous and oxygen-free THF $(1 \mathrm{~mL})$ under $\mathrm{N}_{2}$, then iodine $(12.7 \mathrm{mg}, 0.05 \mathrm{mmol}$, 0.5 equiv.) was added. After stirring at room temperature for $24 \mathrm{~h}$, the mixture was filtered through a pad of celite with EtOAc. The filtrate was evaporated under reduced pressure. The residue was purified by flash column chromatography on silica gel to afford 6 as a light white solid $(24.7 \mathrm{mg}, 75 \%$ yield, $99 \%$ ee $), \mathrm{mp} 100{ }^{\circ} \mathrm{C}-101{ }^{\circ} \mathrm{C} .{ }^{1} \mathrm{H}$ NMR (400 MHz, $\left.\mathrm{CDCl}_{3}\right) \delta 7.47-7.38(\mathrm{~m}, 4 \mathrm{H}), 7.39-7.26(\mathrm{~m}, 6 \mathrm{H}), 7.21-7.10(\mathrm{~m}$, $4 \mathrm{H}), 3.70-3.60(\mathrm{~m}, 1 \mathrm{H}), 2.76-2.68(\mathrm{~m}, 1 \mathrm{H}), 2.62-2.54(\mathrm{~m}, 1 \mathrm{H}), 2.34(\mathrm{~s}, 3 \mathrm{H}) .{ }^{13} \mathrm{C}$ NMR (100 MHz, $\left.\mathrm{CDCl}_{3}\right) \delta 138.2,136.8(\mathrm{~d}, J=7.9 \mathrm{~Hz}), 136.7(\mathrm{~d}, J=7.8 \mathrm{~Hz}), 133.1$ $(\mathrm{d}, J=6.0 \mathrm{~Hz}), 133.0,132.8(\mathrm{~d}, J=1.5 \mathrm{~Hz}), 132.6,129.8,129.2(\mathrm{~d}, J=11.2 \mathrm{~Hz})$, $128.8(\mathrm{~d}, J=2.5 \mathrm{~Hz}), 128.7(\mathrm{~d}, J=2.5 \mathrm{~Hz}), 127.2,120.7$ (d, $J=5.8 \mathrm{~Hz}), 35.7$ (d, $J=$ 
$16.8 \mathrm{~Hz}), 34.3(\mathrm{~d}, J=20.8 \mathrm{~Hz}), 21.1 .{ }^{31} \mathrm{P}$ NMR $\left(162 \mathrm{MHz}, \mathrm{CDCl}_{3}\right) \delta$-20.4. HRMS: $\mathrm{m} / \mathrm{z}$ (ESI) calculated $[\mathrm{M}+\mathrm{H}]^{+}$330.1406, measured: 330.1407 .

${ }^{[\alpha]} \mathrm{D}^{26.7}-7.0255\left(\mathrm{c} 0.25, \mathrm{CHCl}_{3}\right)$. HPLC (IC-3, 0.46*15 cm, $3 \mu \mathrm{m}$, hexane/ isopropanol $=9 / 1$, flow $0.7 \mathrm{~mL} / \mathrm{min}$, detection at $214 \mathrm{~nm}$ ) retention time $=7.768 \mathrm{~min}$ (major) and $8.512 \min$ (minor).

\subsection{Synthesis of Chiral Amine-Based Phosphines}
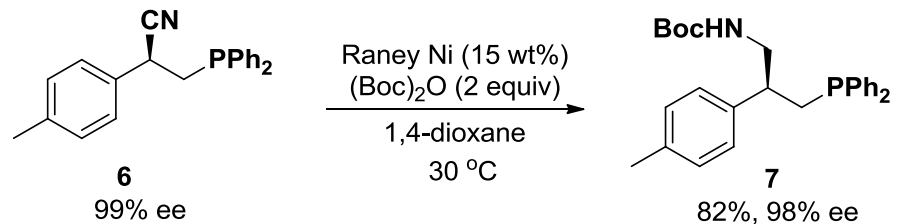

According to the same procedure as 4.1

White solid. mp $120{ }^{\circ} \mathrm{C}-121{ }^{\circ} \mathrm{C}$.

${ }^{1} \mathrm{H}$ NMR (400 MHz, $\left.\mathrm{CDCl}_{3}\right) \delta 7.44-7.38(\mathrm{~m}, 2 \mathrm{H}), 7.37-7.29(\mathrm{~m}, 5 \mathrm{H}), 7.28-7.25$ $(\mathrm{m}, 3 \mathrm{H}), 7.10(\mathrm{~d}, J=7.6 \mathrm{~Hz}, 2 \mathrm{H}), 7.02(\mathrm{~d}, J=7.6 \mathrm{~Hz}, 2 \mathrm{H}), 4.36-4.26(\mathrm{~m}, 1 \mathrm{H}), 3.70$ $-3.52(\mathrm{~m}, 1 \mathrm{H}), 3.35-3.22(\mathrm{~m}, 1 \mathrm{H}), 2.79-2.68(\mathrm{~m}, 1 \mathrm{H}), 2.47-2.40(\mathrm{~m}, 1 \mathrm{H}), 2.32(\mathrm{~s}$, 3H), $1.38(\mathrm{~s}, 9 \mathrm{H}) .{ }^{13} \mathrm{C} \mathrm{NMR}\left(100 \mathrm{MHz}, \mathrm{CDCl}_{3}\right) \delta 155.7,139.2(\mathrm{~d}, J=4.8 \mathrm{~Hz}), 138.2$ $(\mathrm{d}, J=14.1 \mathrm{~Hz}), 136.5,133.1(\mathrm{~d}, J=19.4 \mathrm{~Hz}), 132.5(\mathrm{~d}, J=18.6 \mathrm{~Hz}), 129.4,128.8$, $128.5(\mathrm{~d}, J=6.8 \mathrm{~Hz}), 128.3,128.2,127.7,79.1,47.0,42.9(\mathrm{~d}, J=14.5 \mathrm{~Hz}), 33.5(\mathrm{~d}, J$ $=11.3 \mathrm{~Hz}), 28.3,21.0 .{ }^{31} \mathrm{P} \mathrm{NMR}\left(162 \mathrm{MHz}, \mathrm{CDCl}_{3}\right) \delta-21.7 \mathrm{HRMS}: \mathrm{m} / \mathrm{z}(\mathrm{EI})$ calculated $[\mathrm{M}+\mathrm{H}]^{+}$434.2243, measured: 434.2244.

${ }^{[\alpha]} \mathrm{D}^{26.7}-18.4786\left(\mathrm{c} 0.5, \mathrm{CHCl}_{3}\right)$. HPLC (IA, $0.46 * 25 \mathrm{~cm}, 3 \mu \mathrm{m}$, hexane/ isopropanol = 9/1, flow $0.7 \mathrm{~mL} / \mathrm{min}$, detection at $214 \mathrm{~nm}$ ) retention time $=10.427$ min (major) and $9.477 \mathrm{~min}$ (minor).
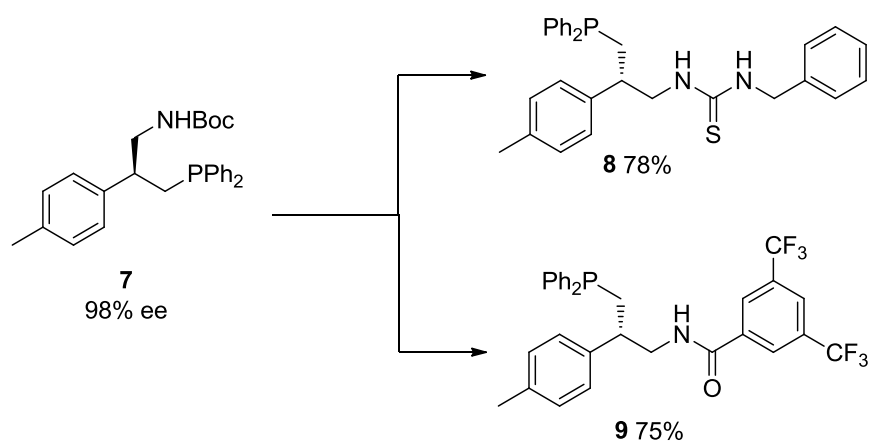
To a $10 \mathrm{~mL}$ sealed tube, 7 (43.3 $\mathrm{mg}, 0.1 \mathrm{mmol})$ was dissolved in degassed mixed solvent DCM/TFA $(2: 1,2 \mathrm{~mL})$ under $\mathrm{N}_{2}$. After stirring at room temperature for $2 \mathrm{~h}$, degassed aqueous saturated $\mathrm{NaHCO}_{3}$ solution $(20 \mathrm{~mL})$ was added to the reaction mixture, and the aqueous phase was extracted with DCM $(3 \times 20 \mathrm{~mL})$. The combined organic phase was washed with brine, dried over anhydrous $\mathrm{Na}_{2} \mathrm{SO}_{4}$ and concentrated in vacuum, the crude product was used directly for the next step.

To a $10 \mathrm{~mL}$ sealed tube, the crude product was dissolved in anhydrous and degassed DCM (2 mL) under $\mathrm{N}_{2}$, then benzyl isothiocyanate was added. After stirring at room temperature for $4 \mathrm{~h}, 5 \%$ aqueous hydrochloric acid $(10 \mathrm{~mL})$, and the aqueous phase was extracted with DCM $(3 \times 20 \mathrm{~mL})$. The combined organic phase was washed with brine, dried over anhydrous $\mathrm{Na}_{2} \mathrm{SO}_{4}$ and concentrated in vacuum, the residue was purified by flash column chromatography on silica gel to afford $\mathbf{8}$.

To a $10 \mathrm{~mL}$ sealed tube, the crude product was dissolved in anhydrous and degassed DCM (2 mL) under $\mathrm{N}_{2}$, then $\mathrm{Et}_{3} \mathrm{~N}$ (2.0 eq) and $\operatorname{ArCOCl}(1.2 \mathrm{eq})$ were added. After stirring at room temperature for $4 \mathrm{~h}$, saturated $\mathrm{NaHCO}_{3}$ solution $(20 \mathrm{~mL})$ was added to the reaction mixture, and the aqueous phase was extracted with DCM $(3 \times 20$ $\mathrm{mL})$. The combined organic phase was washed with brine, dried over anhydrous $\mathrm{Na}_{2} \mathrm{SO}_{4}$ and concentrated in vacuum, the residue was purified by flash column chromatography on silica gel to afford 9.

8 overall $78 \%$ yields with two steps, ${ }^{1} \mathrm{H}$ NMR $\left(400 \mathrm{MHz}, \mathrm{CDCl}_{3}\right) \delta 7.41-7.35(\mathrm{~m}$, 2H), $7.35-7.23(\mathrm{~m}, 11 \mathrm{H}), 7.20-7.10(\mathrm{~m}, 2 \mathrm{H}), 7.07(\mathrm{~d}, J=7.6 \mathrm{~Hz}, 2 \mathrm{H}), 6.95(\mathrm{~d}, J=$ $7.6 \mathrm{~Hz}, 2 \mathrm{H}), 6.48-5.82(\mathrm{~m}, 1 \mathrm{H}), 5.80-5.26(\mathrm{~m}, 1 \mathrm{H}), 4.66-3.20(\mathrm{~m}, 2 \mathrm{H}), 4.19-$ $3.83(\mathrm{~m}, 1 \mathrm{H}), 3.61-3.43(\mathrm{~m}, 1 \mathrm{H}), 2.87-2.77(\mathrm{~m}, 1 \mathrm{H}), 2.45-2.25(\mathrm{~m}, 2 \mathrm{H}), 2.31(\mathrm{~s}$, $3 \mathrm{H}) .{ }^{13} \mathrm{C}$ NMR $\left(100 \mathrm{MHz}, \mathrm{CDCl}_{3}\right) \delta 181.6,138.6(\mathrm{~d}, J=5.4 \mathrm{~Hz}), 138.0(\mathrm{~d}, J=11.4$ $\mathrm{Hz}), 137.8$ (d, $J=12.9 \mathrm{~Hz}), 136.8,132.8$ (d, $J=19.0 \mathrm{~Hz}), 132.5$ (d, $J=19.0 \mathrm{~Hz})$, $129.6,128.8,128.7,128.5,128.5,128.4,128.3,127.7,127.4,50.9,48.0,41.9(\mathrm{~d}, J=$ 15.2 Hz), 33.3 (d, $J=12.9 \mathrm{~Hz}), 21.0 .{ }^{31} \mathrm{P}$ NMR (162 MHz, $\left.\mathrm{CDCl}_{3}\right) \delta$-21.1. HRMS: $\mathrm{m} / \mathrm{z}$ (ESI) calculated $[\mathrm{M}+\mathrm{H}]^{+}$483.2018, measured: 483.2020.

9 overall $75 \%$ yields with two steps, ${ }^{1} \mathrm{H}$ NMR $\left(400 \mathrm{MHz}, \mathrm{CDCl}_{3}\right) \delta 8.12-7.82(\mathrm{~m}$, 
3H), $7.55-7.27(\mathrm{~m}, 10 \mathrm{H}), 7.20-7.00(\mathrm{~m}, 4 \mathrm{H}), 4.07-3.95(\mathrm{~m}, 1 \mathrm{H}), 3.63-3.53(\mathrm{~m}$, 1H), $3.01-2.85(\mathrm{~m}, 1 \mathrm{H}), 2.56-2.43(\mathrm{~m}, 2 \mathrm{H}), 2.34(\mathrm{~s}, 3 \mathrm{H}) .{ }^{13} \mathrm{C}$ NMR (100 MHz, $\left.\mathrm{CDCl}_{3}\right) \delta 167.5,143.4,139.0,138.9,138.6(\mathrm{~d}, J=13.6 \mathrm{~Hz}), 136.8,133.1(\mathrm{~d}, J=19.5$ $\mathrm{Hz}), 132.5(\mathrm{~d}, J=18.4 \mathrm{~Hz}), 131.3(\mathrm{q}, J=33.6 \mathrm{~Hz}), 129.2,128.9,128.5$ (d, $J=7.0 \mathrm{~Hz})$, 128.4, $128.4(\mathrm{~d}, J=6.5 \mathrm{~Hz}), 128.0,127.5,124.2,123.2,121.5,116.7,52.2,40.8(\mathrm{~d}, J$ $=15.2 \mathrm{~Hz}), 33.8(\mathrm{~d}, J=14.1 \mathrm{~Hz}), 20.8 .{ }^{31} \mathrm{P} \mathrm{NMR}\left(162 \mathrm{MHz}, \mathrm{CDCl}_{3}\right) \delta-20.8 \mathrm{HRMS}$ : $\mathrm{m} / \mathrm{z}(\mathrm{ESI})$ calculated $[\mathrm{M}+\mathrm{H}]^{+}$574.1729, measured: 574.1732 .

\section{Mechanistic Studies}

\subsection{Synthesis of ${ }^{t} \mathrm{BuOOSiMe}_{3}$}

To a $250 \mathrm{~mL}$ sealed tube, TMSCN (10 mmol) was dissolved in anhydrous DCM (20 mL) under $\mathrm{N}_{2}$, then ${ }^{t} \mathrm{BuOOH}(10 \mathrm{mmol}, 5.0 \mathrm{~mol} / \mathrm{L}$ in decane) was dropwise added in $10 \mathrm{~min}$, and the mixture was stirred at $0{ }^{\circ} \mathrm{C}$ for $2 \mathrm{~h}$. After reaction completion, saturated $\mathrm{Na}_{2} \mathrm{CO}_{3}$ solution $(100 \mathrm{~mL})$ was added to the reaction mixture, and the aqueous phase was extracted with DCM $(3 \times 50 \mathrm{~mL})$. The combined organic phase was washed with brine, dried over anhydrous $\mathrm{Na}_{2} \mathrm{SO}_{4}$ and concentrated in vacuum. The crude product ${ }^{t} \mathrm{BuOOTMS}\left(1.5 \mathrm{~g}, 97 \%\right.$ ) was used directly. ${ }^{1} \mathrm{H}$ NMR (400 MHz, $\left.\mathrm{CDCl}_{3}\right) \delta 1.218(\mathrm{~s}, 9 \mathrm{H}), 0.175(\mathrm{~s}, 9 \mathrm{H}) .{ }^{13} \mathrm{C} \mathrm{NMR}\left(100 \mathrm{MHz}, \mathrm{CDCl}_{3}\right) \delta 80.2,26.2,1.1$. HRMS: m/z (EI) calculated [M] ${ }^{+}$162.1076, measured: 162.1072.

Cautious! Owing to the generation of $\mathrm{HCN}$, the reaction should be conducted in the fume hood, and the aqueous phase should be treated carefully and quenched by aqueous $\mathrm{KMnO}_{4}$ or $\mathrm{NaOCl}$.

\subsection{Control Experiments of ${ }^{t} \mathrm{BuOOH}$ and ${ }^{t} \mathrm{BuOOSiMe}_{3}$}




$$
\begin{aligned}
& \underset{1: 1 \text { mol ratio }}{\mathrm{TMSCN}}+\underset{\mathrm{H}}{\mathrm{H}_{2} \mathrm{O}} \underset{\mathrm{CDCl}}{\mathrm{C} 0.5 \mathrm{~h}} \mathrm{HCN}+\mathrm{TMSOH} \\
& \underset{1: 1 \mathrm{~mol} \text { ratio }}{\mathrm{TMSCN}}+{ }^{{ }^{t} \mathrm{BuOOH} \text { (in water) }} \underset{\mathrm{rt}, 0.5 \mathrm{~h}}{\stackrel{\mathrm{dry} \mathrm{CDCl}_{3}}{\longrightarrow}} \\
& \mathrm{HCN}+\mathrm{TMSOH}+{ }^{t} \mathrm{BuOOSiMe}{ }_{3}+{ }^{t} \mathrm{BuOOH} \\
& 100 \% \text { trace }>95 \%
\end{aligned}
$$

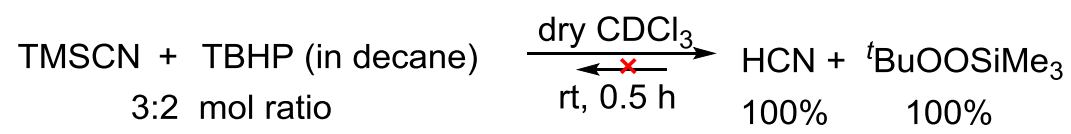

Reaction a: To a dried sealed NMR tube by rubber septum, TMSCN $(0.1 \mathrm{mmol})$ was dissolved in anhydrous $\mathrm{CDCl}_{3}(0.5 \mathrm{~mL})$ under $\mathrm{N}_{2}$, then $\mathrm{H}_{2} \mathrm{O}(0.1 \mathrm{mmol}, 1.0$ equiv. $)$ was added to the mixture. The mixture was shook for a couple of minutes and analyzed by ${ }^{1} \mathrm{H}$ NMR.

Reaction b: To a dried sealed NMR tube by rubber septum, TMSCN $(0.1 \mathrm{mmol})$ was dissolved in anhydrous $\mathrm{CDCl}_{3}(0.5 \mathrm{~mL})$ under $\mathrm{Ar}$, then ${ }^{t} \mathrm{BuOOH}(0.1 \mathrm{mmol}, 1.0$ equiv., $70 \%$ in water) was added to the mixture. The mixture was shook for a couple of minutes and analyzed by ${ }^{1} \mathrm{H}$ NMR and GC-MS.

Reaction c: (1) To a dried sealed NMR tube by rubber septum, TMSCN (0.3 mmol) was dissolved in anhydrous $\mathrm{CDCl}_{3}(0.5 \mathrm{~mL})$ under $\mathrm{Ar}$, then ${ }^{t} \mathrm{BuOOH}(0.2 \mathrm{mmol}, 0.75$ equiv., $5.0 \mathrm{~mol} / \mathrm{L}$ in decane) was added to the mixture. The mixture was shook for a couple of minutes and analyzed by ${ }^{1} \mathrm{H}$ NMR.

(2) To a dried sealed NMR tube by rubber septum, tBuOOTMS (0.2 mmol) was dissolved in degassed $\mathrm{CDCl}_{3}(0.5 \mathrm{~mL})$ under Ar, then TMSCN (0.2 mmol, 1.0 equiv) and $\mathrm{H}_{2} \mathrm{O}(0.3 \mathrm{mmol}, 1.5 \mathrm{eq})$ were added to the mixture. The mixture was shook for a couple of minutes and analyzed by ${ }^{1} \mathrm{H}$ NMR.

\section{Reaction a}




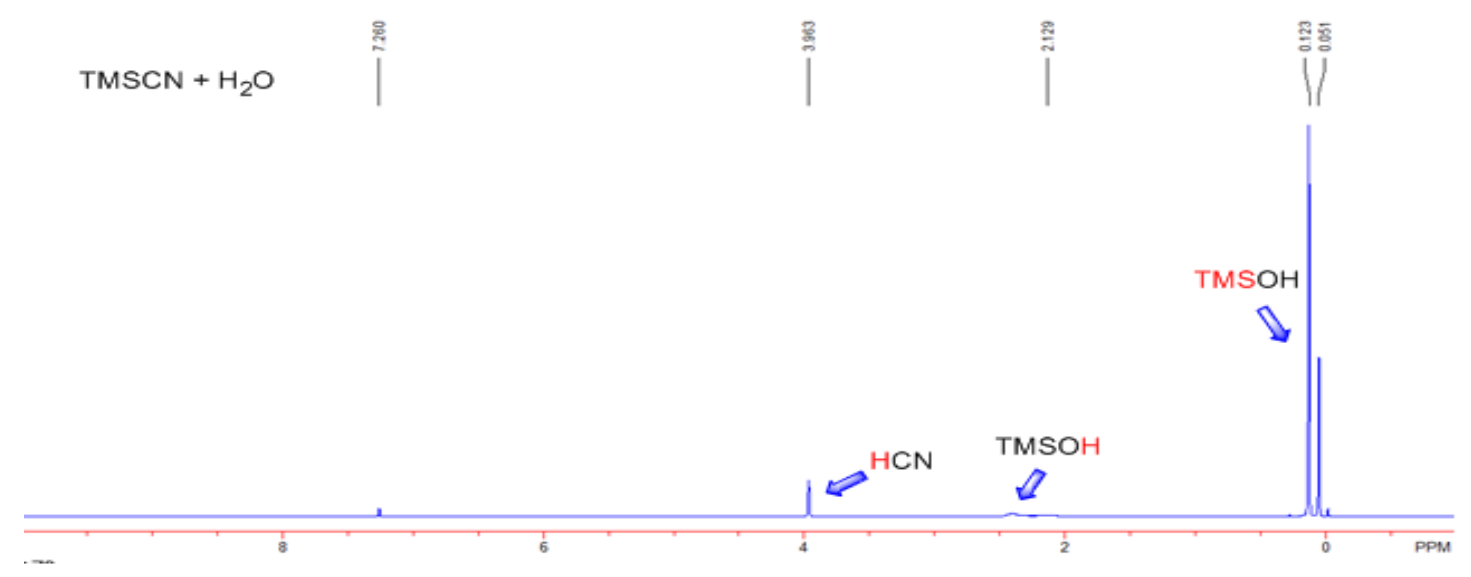

\section{Reaction b}

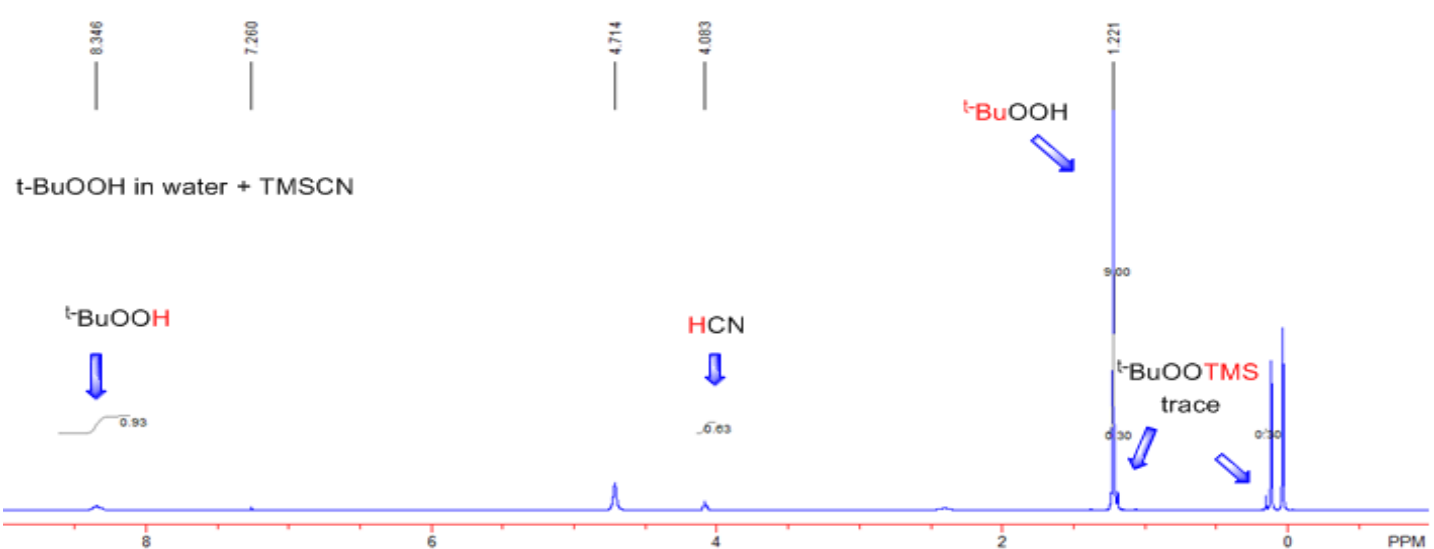

\section{Reaction c}

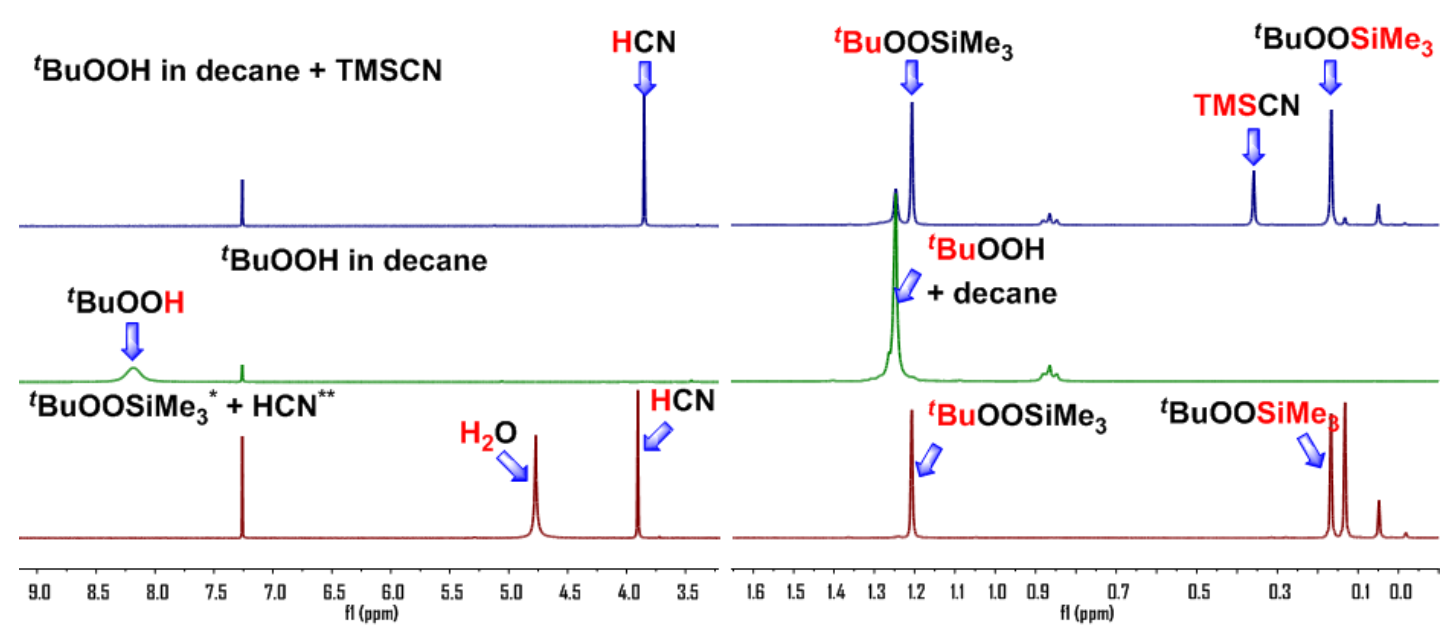

Figure S1. The ${ }^{1} \mathrm{H}$ NMR spectrum of the reactions of ${ }^{t} \mathrm{BuOOH}$ and ${ }^{t} \mathrm{BuOOSiMe}_{3}$. ${ }^{*}{ }^{t} \mathrm{BuOOSiMe} \mathrm{S}_{3}$ was synthesized; $* * \mathrm{HCN}$ was generated from TMSCN and $\mathrm{H}_{2} \mathrm{O}$. 
According to ${ }^{1} \mathrm{H}$ NMR spectrum, we concluded that both water and TBHP reacted with TMSCN to release $\mathrm{HCN}$ (a, b), and water would preferentially react with TMSCN (c). A new species was generated in the reaction of TMSCN and TBHP (in decane), which was proposed to be ${ }^{t}$ BuOOTMS.

\subsection{Control Experiments}
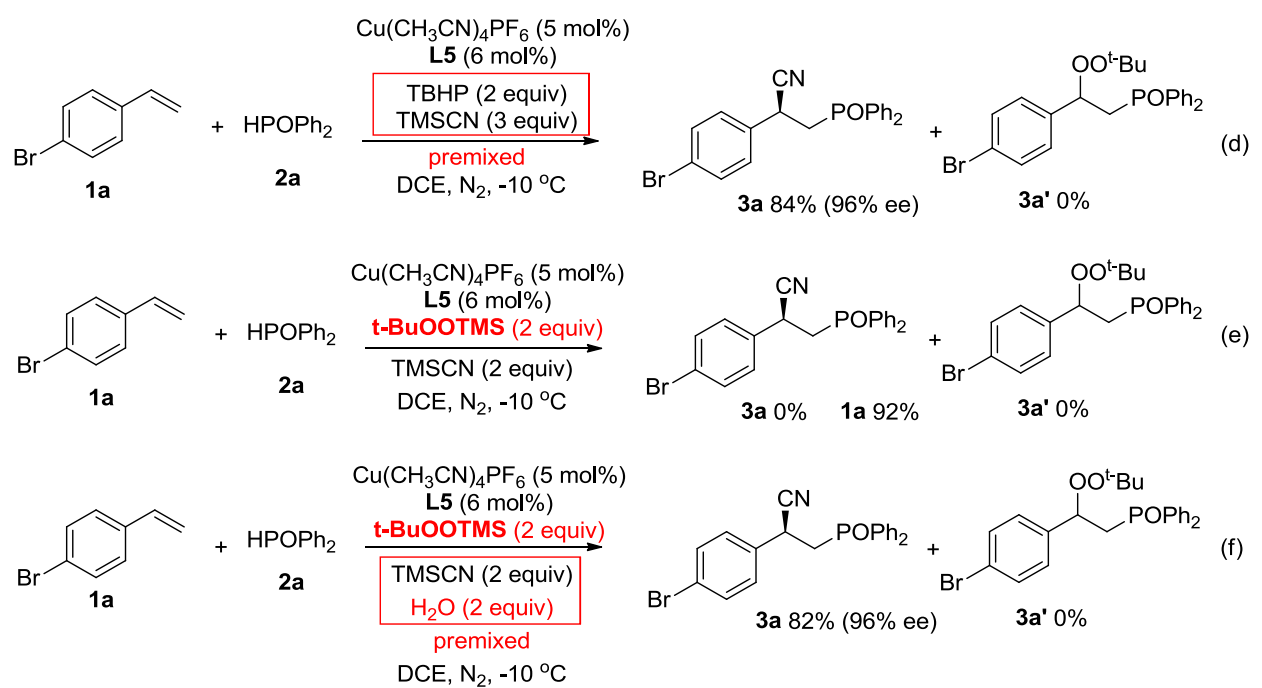

Reaction d: The ${ }^{t} \mathrm{BuOOH}$ ( 2 equiv.) and TMSCN (3 equiv.) were premixed, and ${ }^{t} \mathrm{BuOOH}$ was completely converted to ${ }^{t} \mathrm{BuOOSiMe}_{3}$. Then the solution was applied for the reaction of $1 \mathbf{a}$ and $\mathbf{2 a}$ under the general conditions (on a $0.1 \mathrm{mmol}$ scale). The reaction provided the similar results as the standard reaction in Table 1 to give the product 3a as a white solid (34.8 $\mathrm{mg}, 84 \%$ yield, $96 \%$ ee).

Reaction e: The reaction using ${ }^{t} \mathrm{BuOOSiMe}_{3}(0.0324 \mathrm{~g}, 0.2 \mathrm{mmol}, 2.0$ equiv.) instead of ${ }^{t} \mathrm{BuOOH}$ was conducted under the general conditions (on a $0.1 \mathrm{mmol}$ scale). The mixture was analyzed by ${ }^{1} \mathrm{H} \mathrm{NMR}$ with $\mathrm{CH}_{2} \mathrm{Br}_{2}(0.1 \mathrm{mmol})$ as an internal standard, no product 3a was detected and 1a was recovered (92\%).

Reaction f: To a reaction of TMSCN (0.2 mmol, 2.0 equiv.) in DCE, $\mathrm{H}_{2} \mathrm{O}(0.2 \mathrm{mmol}$, 2.0 equiv.) was added by syringe at $0{ }^{\circ} \mathrm{C}$ in a sealed bottle. Then the premixed solution was applied to the reaction of $\mathbf{1 a}$ and $\mathbf{2 a}$ (on a $0.1 \mathrm{mmol}$ scale) at $-10{ }^{\circ} \mathrm{C}$, where ${ }^{t}$ BuOOTMS (0.0324g, $0.2 \mathrm{mmol}, 2.0$ equiv.) was used as oxidant. The reaction 
afforded the product $\mathbf{3 a}$ as a white solid (33.6 mg, $82 \%$ yield, $96 \%$ ee).

These results revealed that ${ }^{t} B u O O T M S$ was the true oxidant, and $H C N$ acted as a real cyanide source to participate in the cyanation of benzylic radicals and excessive amounts of HCN did not poison copper catalysts presumably due to a small dissociation constant of $\mathrm{HCN}\left(\mathrm{HCN}\right.$ in $\left.\mathrm{H}_{2} \mathrm{O}, \mathrm{pKa}=9.2\right)$, which were distinctly different from our previous studies of the cyanide effect.

\subsection{Reaction with ${ }^{t} \mathrm{BuOO}^{t} \mathrm{Bu}$ as Oxidant}

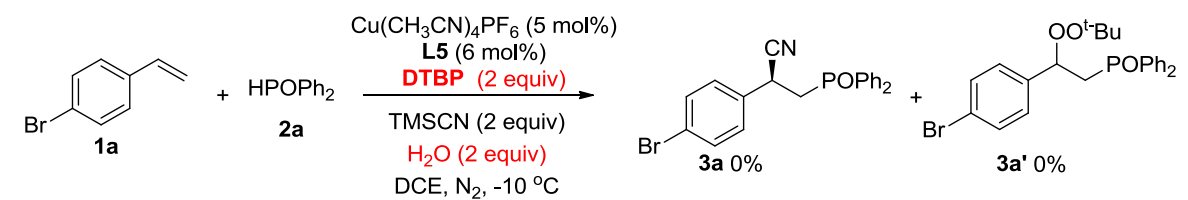

When ${ }^{t} \mathrm{BuOO}{ }^{t} \mathrm{Bu}$ instead of ${ }^{t} \mathrm{BuOOH}$ was used as oxidant for the reaction of $\mathbf{1 a}$ and $2 \mathbf{a}$ under the standard conditions, no reaction occurred. The reaction did not work either by replacing the pure TMSCN with the premixed TMSCN and $\mathrm{H}_{2} \mathrm{O}$. These observations revealed that ${ }^{t} \mathrm{BuOO}{ }^{t} \mathrm{Bu}$ was ineffective for the reaction.

\subsection{Radical Trapping Reaction}
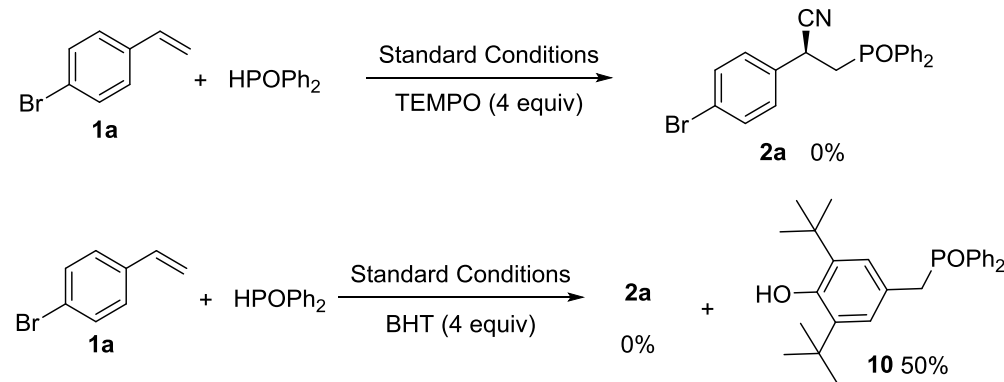

These reactions were conducted on a $0.1 \mathrm{mmol}$ scale according to the general procedure. To a $10 \mathrm{~mL}$ sealed tube, ligand $\mathbf{L 5}$ (6.2 $\mathrm{mg}, 0.012 \mathrm{mmol}, 6 \mathrm{~mol} \%)$ and $\mathrm{Cu}\left(\mathrm{CH}_{3} \mathrm{CN}\right)_{4} \mathrm{PF}_{6}(3.7 \mathrm{mg}, 0.010 \mathrm{mmol}, 5 \mathrm{~mol} \%)$ were dissolved in anhydrous and oxygen-free DCE $(2.0 \mathrm{~mL})$ under Ar. The colorless solution was stirred at $-10{ }^{0} \mathrm{C}$ for 15 mins, and then substrate 1a (36.4 mg, $0.2 \mathrm{mmol}, 1.0$ equiv.), TMSCN (52 uL, 0.4 
mmol, 2.0 equiv.), $\mathrm{HPOPh}_{2} \mathbf{2 a}(80.8 \mathrm{mg}, 0.4 \mathrm{mmol}, 2.0$ equiv.), TEMPO (4.0 equiv.) or BHT (4.0 equiv.) and ${ }^{t} \mathrm{BuOOH}(72.0 \mathrm{uL}, 0.4 \mathrm{mmol}, 2.0$ equiv., $5.5 \mathrm{~mol} / \mathrm{L}$ in $\mathrm{N}$-decane) were sequentially added at $-10{ }^{\circ} \mathrm{C}$. When ${ }^{t} \mathrm{BuOOH}$ was added, the reaction mixture turned purple. After that, the mixture was stirred at $-10{ }^{\circ} \mathrm{C}$ for $12 \mathrm{~h}$. After reaction completion, the solvent was evaporated under reduced pressure. The mixture was analyzed by ${ }^{1} \mathrm{H}-\mathrm{NMR}$ with $\mathrm{CH}_{2} \mathrm{Br}_{2}(0.1 \mathrm{mmol})$ as an internal standard. The product 3a was not obtained in the both two reactions, but the product $\mathbf{1 0}$ was obtained in the latter reaction, suggesting the involvement of phosphinoyl radicals in the reaction.

\subsection{Radical Clock Experiment}

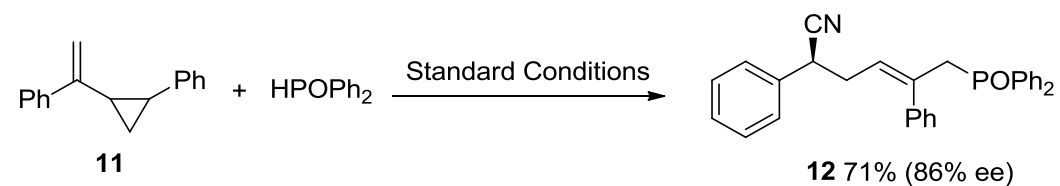

To a $10 \mathrm{~mL}$ sealed tube, ligand $\mathbf{L 5}(3.1 \mathrm{mg}, 0.006 \mathrm{mmol}, 6 \mathrm{~mol} \%)$ and $\mathrm{Cu}\left(\mathrm{CH}_{3} \mathrm{CN}\right)_{4} \mathrm{PF}_{6}(1.8 \mathrm{mg}, 0.005 \mathrm{mmol}, 5 \mathrm{~mol} \%)$ were dissolved in anhydrous and oxygen-free DCE $(0.95 \mathrm{~mL})$ under Ar. The colorless solution was stirred at $-10{ }^{0} \mathrm{C}$ for 15 mins, then substrate 11 (22 mg, 0.1 mmol, 1.0 equiv.), $\mathrm{HPOPh}_{2} \mathbf{2 a}(40.4 \mathrm{mg}, 0.2$ mmol, 2.0 equiv.) and ${ }^{t} \mathrm{BuOOH}(72.0 \mu \mathrm{L}, 0.4 \mathrm{mmol}, 2.0$ equiv., $5.5 \mathrm{~mol} / \mathrm{L}$ in $N$-decane) were sequentially added at $-10{ }^{\circ} \mathrm{C}$. After that, the mixture was stirred at $-10{ }^{\circ} \mathrm{C}$ for 12 $\mathrm{h}$ before the reaction mixture turned colorless. After reaction completion, $5 \% \mathrm{Na}_{2} \mathrm{~S}_{2} \mathrm{O}_{3}$ cooled aqueous solution $(2 \mathrm{~mL})$ were added, and the reaction mixture was stirred for 30 mins. Then the aqueous phase was extracted with DCM $(3 \times 10 \mathrm{~mL})$. The combined organic phase was washed with brine, dried over anhydrous $\mathrm{Na}_{2} \mathrm{SO}_{4}$ and concentrated in vacuum. The residue was purified by flash column chromatography on silica gel with a gradient of petroleum ether/acetone (4:1) to afford the product $\mathbf{1 2}$ as a white solid (31.2 mg, $71 \%$ yield, $86 \%$ ee), mp $134{ }^{\circ} \mathrm{C}-135{ }^{\circ} \mathrm{C} .{ }^{1} \mathrm{H} \mathrm{NMR}\left(400 \mathrm{MHz}, \mathrm{CDCl}_{3}\right)$ $\delta 7.66-7.58(\mathrm{~m}, 2 \mathrm{H}), 7.57-7.51(\mathrm{~m}, 2 \mathrm{H}), 7.47-7.41(\mathrm{~m}, 3 \mathrm{H}), 7.41-7.30(\mathrm{~m}, 6 \mathrm{H})$, $7.29-7.22(\mathrm{~m}, 2 \mathrm{H}), 7.11-7.02(\mathrm{~m}, 3 \mathrm{H}), 6.99-6.92(\mathrm{~m}, 2 \mathrm{H}), 5.84(\mathrm{dd}, J=13.6,7.6$ $\mathrm{Hz}, 1 \mathrm{H}), 4.21(\mathrm{dd}, J=16.4,6.4 \mathrm{~Hz}, 1 \mathrm{H}), 3.60-2.45(\mathrm{~m}, 2 \mathrm{H}), 2.95-2.74(\mathrm{~m}, 2 \mathrm{H})$. 
${ }^{13} \mathrm{C}$ NMR $\left(100 \mathrm{MHz}, \mathrm{CDCl}_{3}\right) \delta 142.7(\mathrm{~d}, J=2.4 \mathrm{~Hz}), 135.5,134.3(\mathrm{~d}, J=9.7 \mathrm{~Hz})$, $132.7(\mathrm{~d}, J=97.6 \mathrm{~Hz}), 131.7$ (d, $J=98.1 \mathrm{~Hz}), 131.7$ (d, $J=2.9 \mathrm{~Hz}), 131.8$ (d, $J=2.8$ Hz), 130.9 (d, $J=9.6 \mathrm{~Hz}), 130.7(\mathrm{~d}, J=9.3 \mathrm{~Hz}), 128.9,128.4(\mathrm{~d}, J=11.3 \mathrm{~Hz}), 128.4$ $(\mathrm{d}, J=8.8 \mathrm{~Hz}), 128.2(\mathrm{~d}, J=11.7 \mathrm{~Hz}), 128.04,128.01,127.5,127.0,126.7$ (d, $J=1.2$ $\mathrm{Hz}), 120.8,37.3(\mathrm{~d}, J=3.7 \mathrm{~Hz}), 35.6(\mathrm{~d}, J=2.8 \mathrm{~Hz}), 33.8(\mathrm{~d}, J=65.8 \mathrm{~Hz}) .{ }^{31} \mathrm{P}$ NMR $\left(162 \mathrm{MHz}, \mathrm{CDCl}_{3}\right) \delta 28.6 \mathrm{HRMS}: \mathrm{m} / \mathrm{z}(\mathrm{EI})$ calculated $[\mathrm{M}]^{+}$448.1825, measured: 448.1826.

${ }^{[\alpha]} \mathrm{D}^{28.9} 115.5525\left(\mathrm{c} 0.8, \mathrm{CHCl}_{3}\right)$. HPLC (IC-3, 0.46*15 cm, $3 \mu \mathrm{m}$, hexane/ isopropanol $=6 / 4$, flow $0.7 \mathrm{~mL} / \mathrm{min}$, detection at $214 \mathrm{~nm}$ ) retention time $=48.310 \mathrm{~min}$ (major) and $52.322 \min$ (minor).

\subsection{UV Experiment}

\section{(a) ${ }^{\mathrm{t}} \mathrm{BuOOSiMe}_{3}$ with $\mathrm{Cu}(\mathrm{L5})$}

Preparation of $1.0^{*} 10^{-4} \mathrm{~mol} / \mathrm{L} \mathrm{Cu}(\mathbf{L 5})$ solution $\mathbf{A}$ (the experiment was carried out in a glove box): To a $10 \mathrm{~mL}$ volumetric flask, $\mathrm{Cu}\left(\mathrm{CH}_{3} \mathrm{CN}\right)_{4} \mathrm{PF}_{6}(0.01 \mathrm{mmol})$ and $\mathbf{L 5}(0.012$ mmol) were dissolved in anhydrous and degassed DCE $(10 \mathrm{~mL})$, and then $1 \mathrm{~mL}$ the solution was further diluted ten times with degassed DCE.

Preparation of $1.0 * 10^{-2} \mathrm{~mol} / \mathrm{L}{ }^{t} \mathrm{BuOOSiMe}_{3}$ solution $\mathbf{B}$ (the experiment was carried out in a glove box): To a $10 \mathrm{~mL}$ volumetric flask, ${ }^{t} \mathrm{BuOOSiMe}_{3}(0.1 \mathrm{mmol})$ was dissolved in anhydrous and degassed DCE $(10 \mathrm{~mL})$.

Measurement: To a cuvette, $0.5 \mathrm{~mL}$ the solution $\mathbf{A}$ and different amounts of the solution $\mathrm{B}(0 \mu \mathrm{L}, 2.5 \mu \mathrm{L}, 4.0 \mu \mathrm{L}, 5.0 \mu \mathrm{L}, 10.0 \mu \mathrm{L})$ were respectively mixed and the mixed solutions were measured (see Figure S3). Meantime, the solution of ${ }^{t} \mathrm{BuOOSiMe}_{3}$ and ${ }^{t} \mathrm{BuOO}^{t} \mathrm{Bu}$ were also measured by UV spectroscopy (Figure S2). 

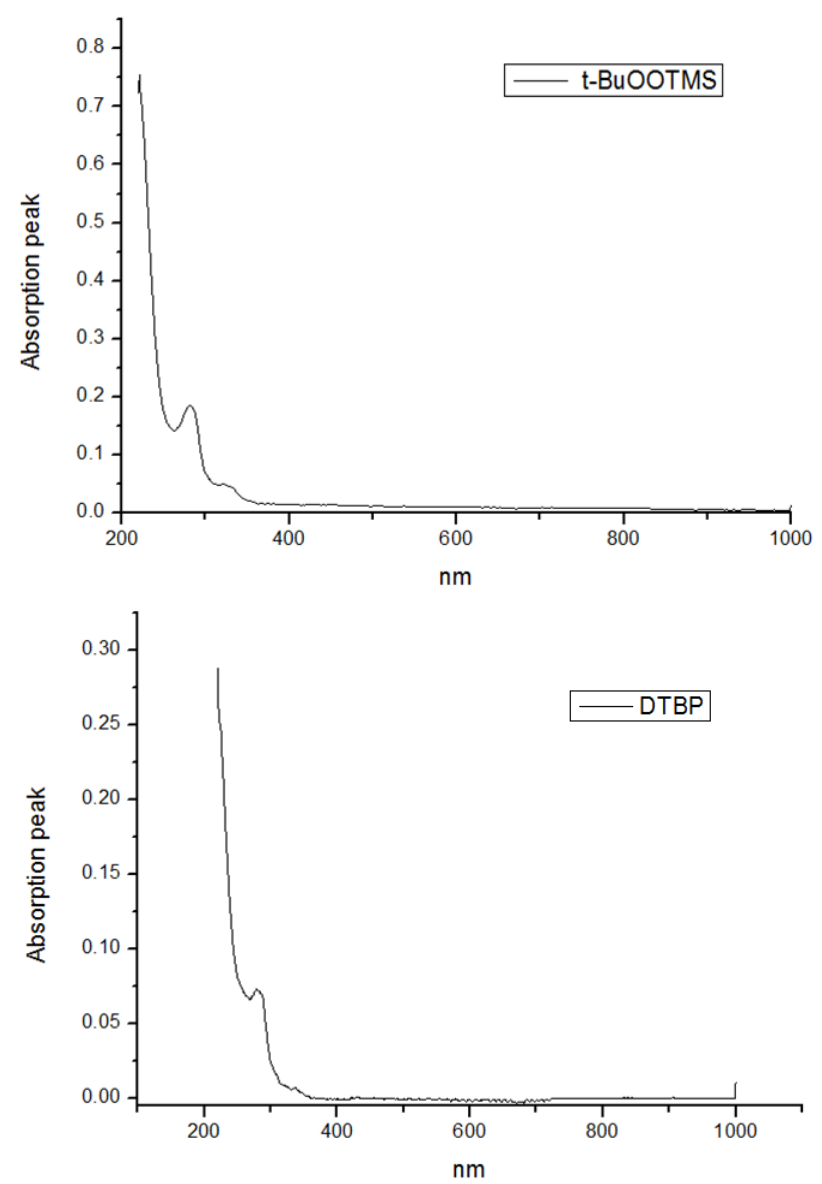

Figure S2. UV spectra of ${ }^{t} \mathrm{BuOOSiMe}_{3}$ (top) and ${ }^{t} \mathrm{BuOO}{ }^{t} \mathrm{Bu}$ (DTBP, bottom).

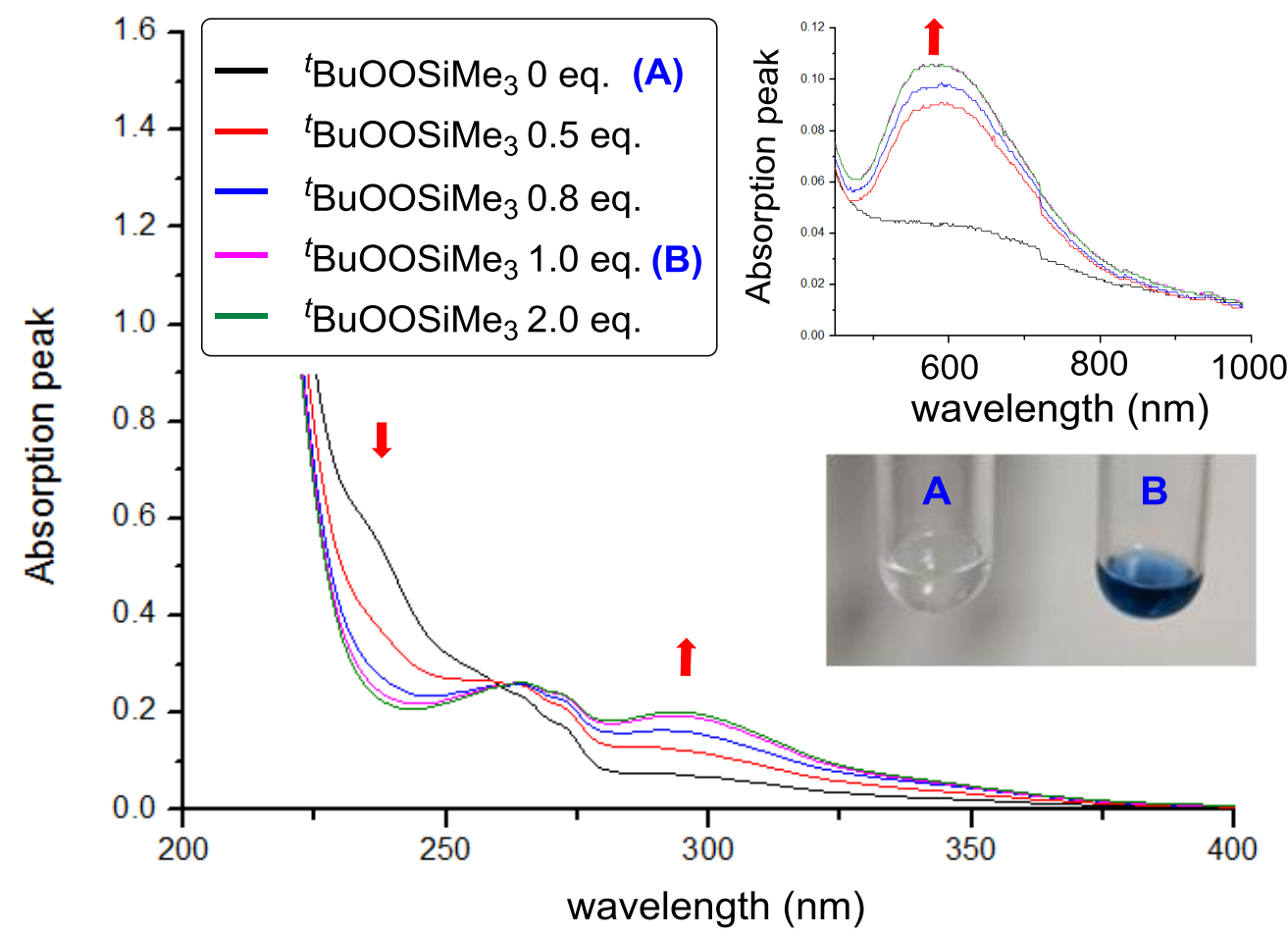

Figure S3. UV spectra of the reactions of $(\mathbf{L 5}) \mathrm{Cu}(\mathrm{I})$ and ${ }^{t} \mathrm{BuOOSiMe}_{3}(0 \sim 2.0$ equiv. $)$. 


\section{(b) ${ }^{t} \mathrm{BuOO}^{t} \mathrm{Bu}$ with $\mathrm{Cu}(\mathrm{L5})$}

Preparation of $1.0 * 10^{-2} \mathrm{~mol} / \mathrm{L}$ DTBP solution $\mathbf{C}$ (the experiment was carried out in a glove box): To a $10 \mathrm{~mL}$ volumetric flask, ${ }^{t} \mathrm{BuOO}{ }^{t} \mathrm{Bu}(0.1 \mathrm{mmol})$ was dissolved in anhydrous and degassed DCE (10 mL).

Measurement: To a cuvette, $0.5 \mathrm{~mL}$ the solution A and different amounts of the solution $\mathrm{C}(0 \mu \mathrm{L}, 2.5 \mu \mathrm{L}, 4.0 \mu \mathrm{L}, 5.0 \mu \mathrm{L}, 10.0 \mu \mathrm{L})$ were respectively mixed and the mixed solutions were measured (see Figure S4).
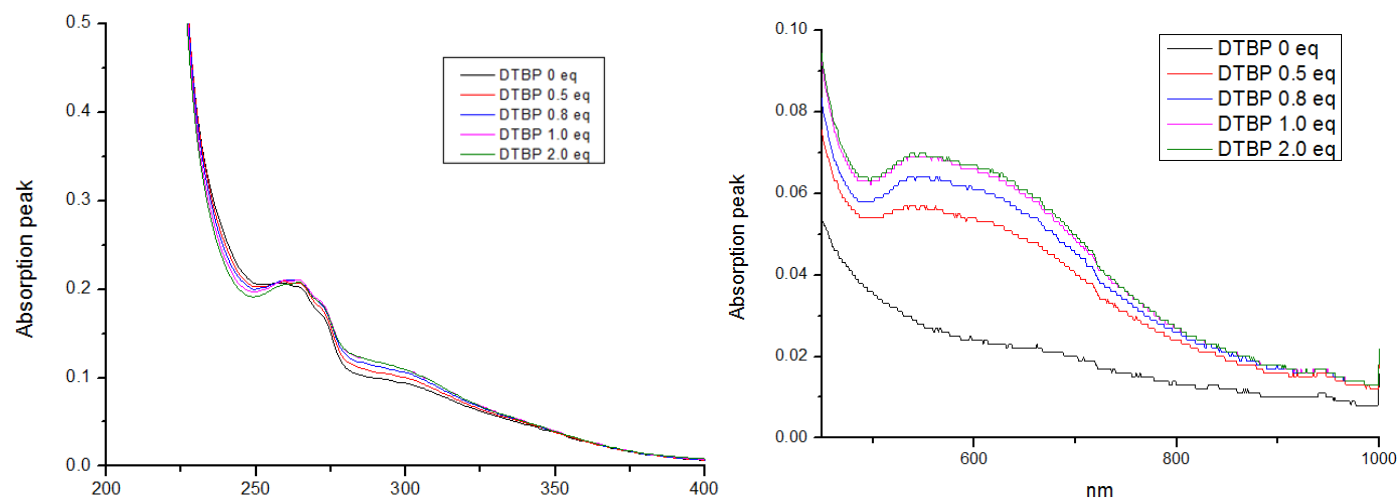

Figure S4. UV spectra of the reactions of $(\mathbf{L 5}) \mathrm{Cu}(\mathrm{I})$ and ${ }^{t} \mathrm{BuOOSiMe}_{3}(0 \sim 2.0$ equiv. $)$.

\subsection{EPR Experiment}

1) To a $10 \mathrm{~mL}$ sealed tube, ligand $\mathbf{L 5}(3.1 \mathrm{mg}, 0.006 \mathrm{mmol})$ and $\mathrm{Cu}\left(\mathrm{CH}_{3} \mathrm{CN}\right)_{4} \mathrm{PF}_{6}(1.8$ $\mathrm{mg}, 0.005 \mathrm{mmol})$ were dissolved in anhydrous and degassed DCE (1 mL) under Ar.

2) To a $10 \mathrm{~mL}$ sealed tube, ligand $\mathbf{L 5}(3.1 \mathrm{mg}, 0.006 \mathrm{mmol})$ and $\mathrm{Cu}\left(\mathrm{CH}_{3} \mathrm{CN}\right)_{4} \mathrm{PF}_{6}(1.8$ $\mathrm{mg}, 0.005 \mathrm{mmol})$ were dissolved in anhydrous and degassed DCE (1 mL) under Ar. After $15 \mathrm{~min},{ }^{t} \mathrm{BuOOTMS}(0.005 \mathrm{mmol})$ was added to the mixture.

3) To a $10 \mathrm{~mL}$ sealed tube, ligand $\mathbf{L 5}(3.1 \mathrm{mg}, 0.006 \mathrm{mmol})$ and $\mathrm{Cu}\left(\mathrm{CH}_{3} \mathrm{CN}\right)_{4} \mathrm{PF}_{6}(1.8$ mg, $0.005 \mathrm{mmol})$ were dissolved in anhydrous and degassed DCE (1 mL) under Ar. After $15 \mathrm{~min},{ }^{t} \mathrm{BuOOTMS}(0.005 \mathrm{mmol})$ and TMSCN $(0.005 \mathrm{mmol})$ were added to the mixture.

4) To a $10 \mathrm{~mL}$ sealed tube, ligand $\mathbf{L 5}(3.1 \mathrm{mg}, 0.006 \mathrm{mmol})$ and $\mathrm{Cu}\left(\mathrm{CH}_{3} \mathrm{CN}\right)_{4} \mathrm{PF}_{6}(1.8$ $\mathrm{mg}, 0.005 \mathrm{mmol})$ were dissolved in anhydrous and degassed DCE (1 mL) under Ar. 
After $15 \mathrm{~min},{ }^{t} \mathrm{BuOOTMS}(0.005 \mathrm{mmol})$, TMSCN $(0.005 \mathrm{mmol})$ and $\mathrm{H}_{2} \mathrm{O}(0.005$ mmol) were added to the mixture. The results of ${ }^{t}$ BuOOTMS listed in Figure S5.

The procedure for ${ }^{t} \mathrm{BuOO}{ }^{t} \mathrm{Bu}$ was same as above (Figure S6).

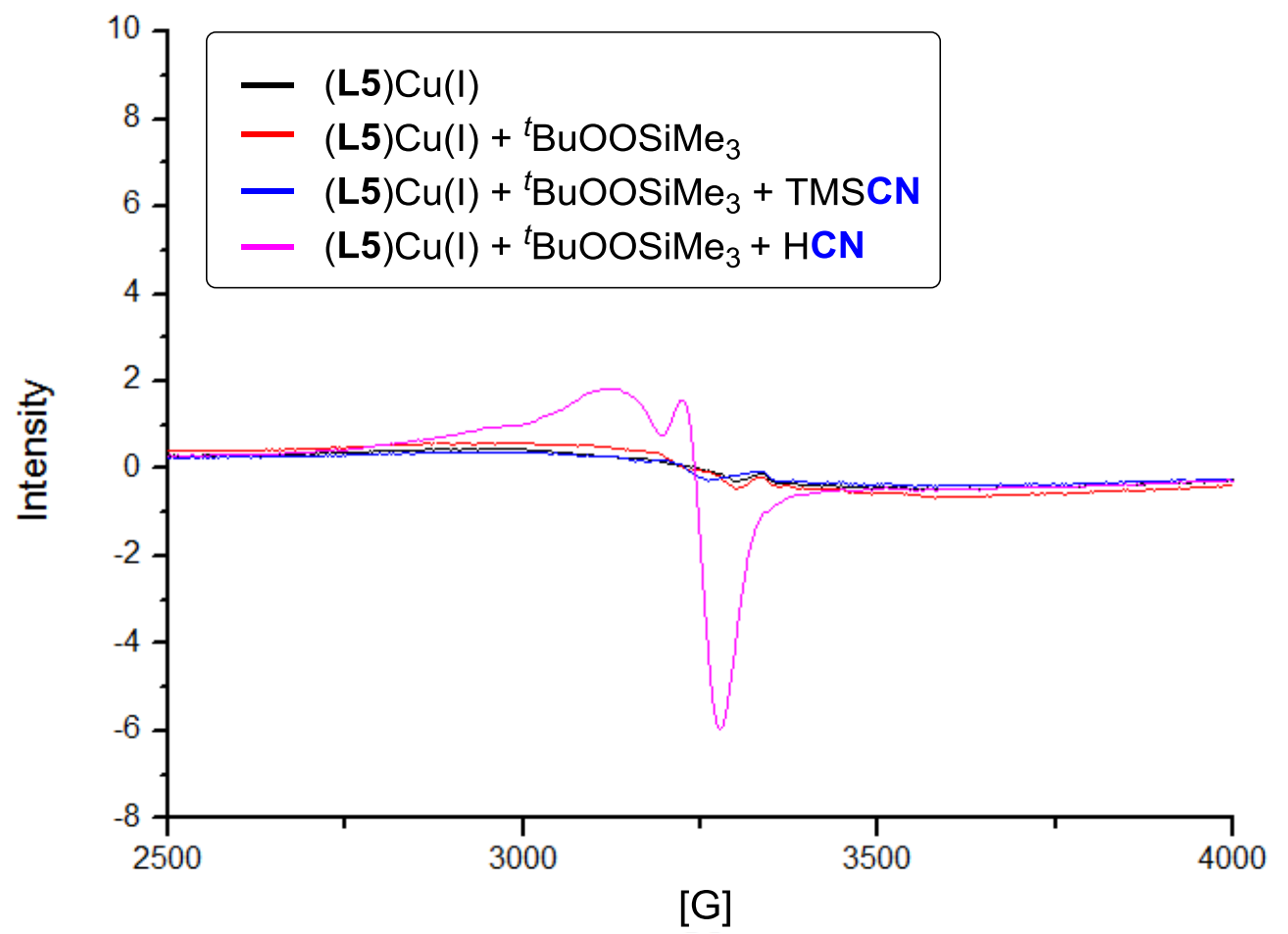

Figure S5. EPR spectra of the reactions of (L5) Cu(I) with ${ }^{t} \mathrm{BuOOSiMe}_{3}$ in the absence or presence of $\mathrm{HCN}$ or TMSCN. 


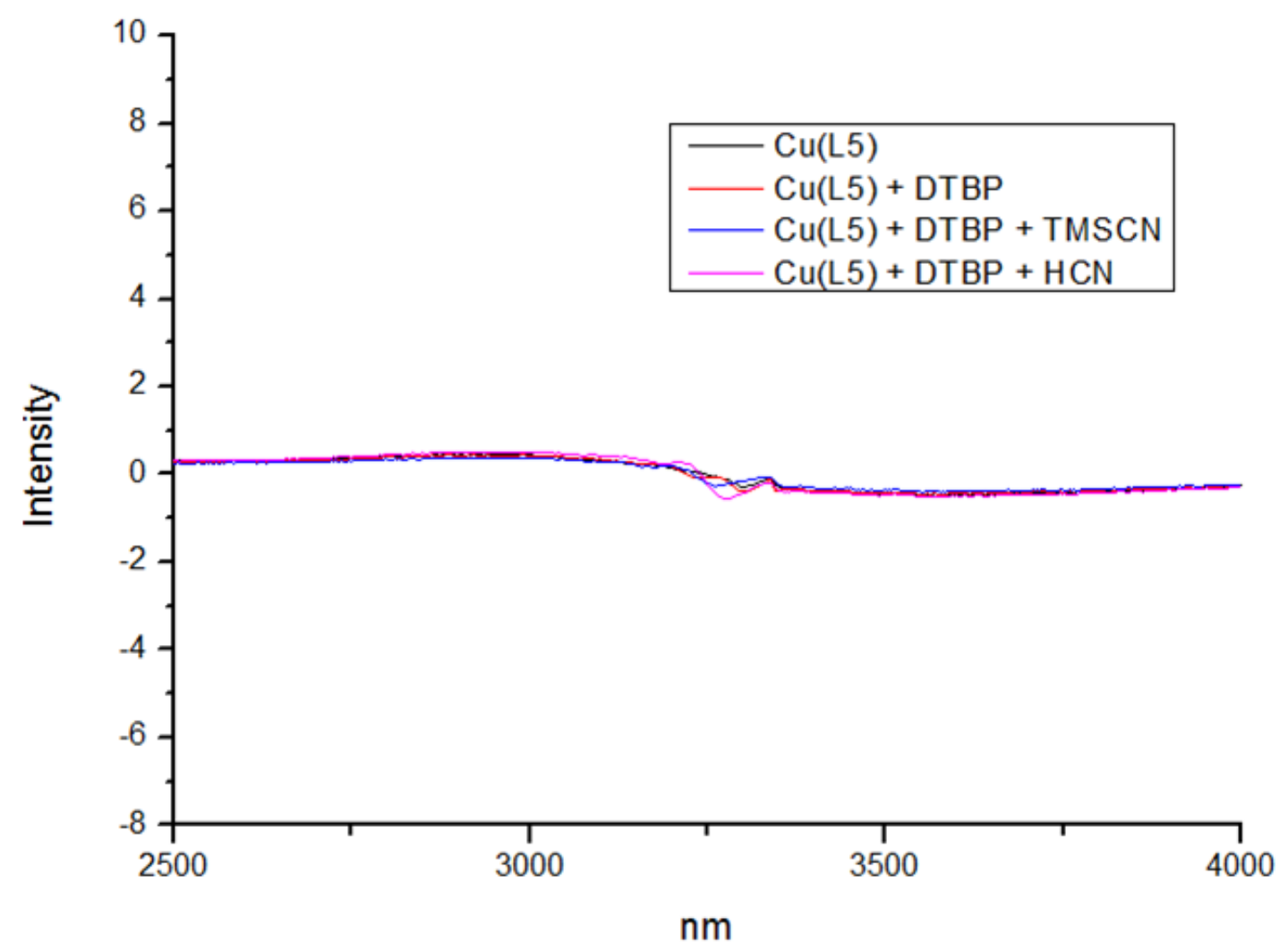

Figure S6. EPR spectra of the reactions of $(\mathbf{L 5}) \mathrm{Cu}(\mathrm{I})$ with ${ }^{t} \mathrm{BuOO}{ }^{t} \mathrm{Bu}$ in the absence or presence of $\mathrm{HCN}$ or TMSCN.

\section{Characterization of the Products}

3a<smiles>N#CC(CPOc1ccccc1)c1ccc(Br)cc1</smiles>

White solid. mp $154{ }^{\circ} \mathrm{C}-155{ }^{\circ} \mathrm{C} .{ }^{1} \mathrm{H}$ NMR (400 MHz, $\left.\mathrm{CDCl}_{3}\right) \delta 7.79-7.72(\mathrm{~m}, 2 \mathrm{H})$, $7.60-7.42(\mathrm{~m}, 6 \mathrm{H}), 7.40-7.27(\mathrm{~m}, 4 \mathrm{H}), 7.16(\mathrm{~d}, J=8.0 \mathrm{~Hz}, 2 \mathrm{H}), 4.50-4.36(\mathrm{~m}$, 1H), $3.07-2.96(\mathrm{~m}, 1 \mathrm{H}), 2.81-2.70(\mathrm{~m}, 1 \mathrm{H}) .{ }^{13} \mathrm{C} \mathrm{NMR}\left(100 \mathrm{MHz}, \mathrm{CDCl}_{3}\right) \delta 133.9$ $(\mathrm{d}, J=6.1 \mathrm{~Hz}), 132.5$ (d, $J=3.0 \mathrm{~Hz}), 132.1,131.9(\mathrm{~d}, J=3.1 \mathrm{~Hz}), 131.5(\mathrm{~d}, J=101.1$ Hz), $131.4(\mathrm{~d}, J=101.1 \mathrm{~Hz}), 130.8(\mathrm{~d}, J=9.2 \mathrm{~Hz}), 130.5$ (d, $J=9.2 \mathrm{~Hz}), 129.3,128.9$ $(\mathrm{d}, J=11.5 \mathrm{~Hz}), 128.6(\mathrm{~d}, J=12.3 \mathrm{~Hz}), 122.7,119.5(\mathrm{~d}, J=10.7 \mathrm{~Hz}), 36.0(\mathrm{~d}, J=$ $67.4 \mathrm{~Hz}), 30.2(\mathrm{~d}, J=2.3 \mathrm{~Hz}) .{ }^{31} \mathrm{P}$ NMR $\left(162 \mathrm{MHz}, \mathrm{CDCl}_{3}\right) \delta 26.8 . \mathrm{HRMS}: \mathrm{m} / \mathrm{z}(\mathrm{ESI})$ calculated $[\mathrm{M}+\mathrm{H}]^{+}$410.0304, measured: 410.0305 . 
${ }^{[\alpha]} \mathrm{D}^{26.4} 82.0106$ (c 1.0, $\mathrm{CHCl}_{3}$ ). HPLC (IC-3, 0.46*15 cm, $3 \mu \mathrm{m}$, hexane/ isopropanol $=6 / 4$, flow $0.7 \mathrm{~mL} / \mathrm{min}$, detection at $214 \mathrm{~nm}$ ) retention time $=10.617 \mathrm{~min}$ (major) and 14.492 min (minor).

3b<smiles>N#C[C@@H](CPOc1ccccc1)c1ccc(Cl)cc1</smiles>

White solid. mp $159{ }^{\circ} \mathrm{C}-160{ }^{\circ} \mathrm{C}$.

${ }^{1} \mathrm{H}$ NMR $\left(400 \mathrm{MHz}, \mathrm{CDCl}_{3}\right) \delta 7.81-7.73(\mathrm{~m}, 2 \mathrm{H}), 7.61-7.44(\mathrm{~m}, 6 \mathrm{H}), 7.39-7.31$ (m, 1H), $7.25(\mathrm{~d}, J=10.4 \mathrm{~Hz}, 2 \mathrm{H}), 7.15(\mathrm{~d}, J=8.4 \mathrm{~Hz}, 2 \mathrm{H}), 4.56-4.45(\mathrm{~m}, 1 \mathrm{H}), 3.08$ $-2.96(\mathrm{~m}, 1 \mathrm{H}), 2.86-2.70(\mathrm{~m}, 1 \mathrm{H}) .{ }^{13} \mathrm{C} \mathrm{NMR}\left(100 \mathrm{MHz}, \mathrm{CDCl}_{3}\right) \delta 134.5,133.4(\mathrm{~d}, J$ $=5.9 \mathrm{~Hz}), 132.5(\mathrm{~d}, J=3.0 \mathrm{~Hz}), 131.9(\mathrm{~d}, J=3.1 \mathrm{~Hz}), 131.5(\mathrm{~d}, J=100.9 \mathrm{~Hz}), 131.4$ $(\mathrm{d}, J=101.0 \mathrm{~Hz}), 130.8(\mathrm{~d}, J=9.9 \mathrm{~Hz}), 130.5$ (d, $J=9.6 \mathrm{~Hz}), 129.2,129.0$ (d, $J=7.3$ $\mathrm{Hz}), 128.9,128.7$ (d, $J=12.2 \mathrm{~Hz}), 119.6(\mathrm{~d}, J=10.6 \mathrm{~Hz}), 36.0(\mathrm{~d}, J=68.4 \mathrm{~Hz}), 30.1$ $(\mathrm{d}, J=1.5 \mathrm{~Hz}) .{ }^{31} \mathrm{P}$ NMR $\left(162 \mathrm{MHz}, \mathrm{CDCl}_{3}\right) \delta 27.7$. HRMS: m/z (EI) calculated $[\mathrm{M}]^{+}$ 365.0736, measured: 365.0740.

${ }^{[\alpha]} \mathrm{D}^{27.9} 253.9040\left(\mathrm{c} 0.4, \mathrm{CHCl}_{3}\right)$. HPLC (IC-3, 0.46*15 cm, $3 \mu \mathrm{m}$, hexane/ isopropanol $=6 / 4$, flow $0.7 \mathrm{~mL} / \mathrm{min}$, detection at $214 \mathrm{~nm}$ ) retention time $=10.328 \mathrm{~min}$ (major) and $13.940 \min$ (minor).

$3 c$<smiles>N#C[C@@H](CPOc1ccccc1)c1ccc(F)cc1</smiles>

White solid. mp $150{ }^{\circ} \mathrm{C}-151{ }^{\circ} \mathrm{C}$.

${ }^{1} \mathrm{H}$ NMR $\left(400 \mathrm{MHz}, \mathrm{CDCl}_{3}\right) \delta 7.80-7.72(\mathrm{~m}, 2 \mathrm{H}), 7.59-7.41(\mathrm{~m}, 6 \mathrm{H}), 7.31-7.37$ $(\mathrm{m}, 2 \mathrm{H}), 7.29-7.20(\mathrm{~m}, 2 \mathrm{H}), 6.88(\mathrm{t}, J=8.4 \mathrm{~Hz}, 2 \mathrm{H}), 4.52-4.40(\mathrm{~m}, 1 \mathrm{H}), 3.06-$ $2.97(\mathrm{~m}, 1 \mathrm{H}), 2.83-2.73(\mathrm{~m}, 1 \mathrm{H}) .{ }^{13} \mathrm{C} \mathrm{NMR}\left(100 \mathrm{MHz}, \mathrm{CDCl}_{3}\right) \delta 162.5(\mathrm{~d}, J=247.4$ Hz), 132.5 (d, $J=3.5 \mathrm{~Hz}), 132.0(\mathrm{~d}, J=3.2 \mathrm{~Hz}), 131.6$ (d, $J=101.0 \mathrm{~Hz}), 131.5$ (d, $J$ = 101.0 Hz), $130.8(\mathrm{~d}, J=9.4 \mathrm{~Hz}), 130.7(\mathrm{~d}, J=6.0 \mathrm{~Hz}), 130.5(\mathrm{~d}, J=9.8 \mathrm{~Hz}), 129.5$ $(\mathrm{d}, J=8.3 \mathrm{~Hz}), 128.9(\mathrm{~d}, J=11.0 \mathrm{~Hz}), 128.6(\mathrm{~d}, J=12.2 \mathrm{~Hz}), 119.8(\mathrm{~d}, J=10.7 \mathrm{~Hz})$, 
$116.0(\mathrm{~d}, J=22.1 \mathrm{~Hz}), 36.1(\mathrm{~d}, J=67.5 \mathrm{~Hz}), 30.0(\mathrm{~d}, J=1.5 \mathrm{~Hz}) .{ }^{31} \mathrm{P} \mathrm{NMR}(162 \mathrm{MHz}$, $\left.\mathrm{CDCl}_{3}\right) \delta$ 27.7. HRMS: m/z (EI) calculated [M] ${ }^{+}$349.1032, measured: 349.1038.

${ }^{[\alpha]} \mathrm{D}^{27.7} 112.7053$ (c 1.0, $\mathrm{CHCl}_{3}$ ). HPLC (IC-3, 0.46*15 cm, $3 \mu \mathrm{m}$, hexane/ isopropanol $=6 / 4$, flow $0.7 \mathrm{~mL} / \mathrm{min}$, detection at $214 \mathrm{~nm}$ ) retention time $=9.962 \mathrm{~min}$ (major) and 12.647 min (minor).

3d<smiles>N#CC(CPO)c1ccccc1</smiles>

White solid. mp $145{ }^{\circ} \mathrm{C}-146{ }^{\circ} \mathrm{C}$.

${ }^{1} \mathrm{H}$ NMR (400 MHz, $\left.\mathrm{CDCl}_{3}\right) \delta 7.84-7.74(\mathrm{~m}, 2 \mathrm{H}), 7.63-7.48(\mathrm{~m}, 5 \mathrm{H}), 7.47-7.41$ (m, 1H), $7.39-7.28(\mathrm{~m}, 4 \mathrm{H}), 7.26-7.15(\mathrm{~m}, 3 \mathrm{H}), 4.50-4.40(\mathrm{~m}, 1 \mathrm{H}), 3.08-2.98$ $(\mathrm{m}, 1 \mathrm{H}), 2.83-2.73(\mathrm{~m}, 1 \mathrm{H}) .{ }^{13} \mathrm{C} \mathrm{NMR}\left(100 \mathrm{MHz}, \mathrm{CDCl}_{3}\right) \delta 135.3(\mathrm{~d}, J=7.1 \mathrm{~Hz})$, $132.4(\mathrm{~d}, J=2.8 \mathrm{~Hz}), 131.9(\mathrm{~d}, J=2.8 \mathrm{~Hz}), 131.7(\mathrm{~d}, J=100.8 \mathrm{~Hz}), 131.5$ (d, $J=$ $100.8 \mathrm{~Hz}), 130.9$ (d, $J=9.8 \mathrm{~Hz}), 130.4(\mathrm{~d}, J=9.9 \mathrm{~Hz}), 129.1,128.8(\mathrm{~d}, J=12.2 \mathrm{~Hz})$, 128.7, $128.5(\mathrm{~d}, J=12.2 \mathrm{~Hz}), 127.4,119.8(\mathrm{~d}, J=8.7 \mathrm{~Hz}), 36.1(\mathrm{~d}, J=67.6 \mathrm{~Hz}), 30.4$ $(\mathrm{d}, J=2.4 \mathrm{~Hz}) .{ }^{31} \mathrm{P}$ NMR $(162 \mathrm{MHz}, \mathrm{CDCl} 3) \delta 27.2$. HRMS: $\mathrm{m} / \mathrm{z}$ (EI) calculated $[\mathrm{M}]^{+}$ 331.1126, measured: 331.1133.

${ }^{[\alpha]} \mathrm{D}^{29.0} 79.9751$ (c 1.0, $\mathrm{CHCl}_{3}$ ). HPLC (IC-3, 0.46*15 cm, $3 \mu \mathrm{m}$, hexane/ isopropanol $=6 / 4$, flow $0.7 \mathrm{~mL} / \mathrm{min}$, detection at $214 \mathrm{~nm}$ ) retention time $=13.355 \mathrm{~min}$ (major) and $16.893 \min$ (minor).

$3 e$<smiles>Cc1ccc(C(C#N)CPOc2ccccc2)cc1</smiles>

White solid. mp $130{ }^{\circ} \mathrm{C}-131{ }^{\circ} \mathrm{C}$.

${ }^{1} \mathrm{H}$ NMR $\left(400 \mathrm{MHz}, \mathrm{CDCl}_{3}\right) \delta 7.79(\mathrm{dd}, J=11.6,6.8 \mathrm{~Hz}, 2 \mathrm{H}), 7.60-7.48(\mathrm{~m}, 5 \mathrm{H})$, $7.44(\mathrm{t}, J=7.6 \mathrm{~Hz} 1 \mathrm{H}), 7.34(\mathrm{dt}, J=7.6,2.8 \mathrm{~Hz}, 2 \mathrm{H}), 7.18$ (d, $J=8.0 \mathrm{~Hz}, 2 \mathrm{H}), 7.01$ (d, $J=7.6 \mathrm{~Hz}, 2 \mathrm{H}), 4.46-4.35(\mathrm{~m}, 1 \mathrm{H}), 3.07-2.96(\mathrm{~m}, 1 \mathrm{H}), 2.82-2.71(\mathrm{~m}, 1 \mathrm{H}), 2.25$ (s, 3H). ${ }^{13} \mathrm{C} \mathrm{NMR}\left(100 \mathrm{MHz}, \mathrm{CDCl}_{3}\right) \delta 138.2,132.4(\mathrm{~d}, J=2.5 \mathrm{~Hz}), 132.3(\mathrm{~d}, J=7.5$ 
Hz), 131.8 (d, $J=2.7 \mathrm{~Hz}), 131.7$ (d, $J=101.0 \mathrm{~Hz}), 131.5$ (d, $J=100.2 \mathrm{~Hz}), 130.9$ (d, $J=9.9 \mathrm{~Hz}), 130.5(\mathrm{~d}, J=9.3 \mathrm{~Hz}), 129.7,128.9(\mathrm{~d}, J=11.8 \mathrm{~Hz}), 128.6(\mathrm{~d}, J=11.9$ $\mathrm{Hz}), 127.4,120.0(\mathrm{~d}, J=9.6 \mathrm{~Hz}), 36.2$ (d, $J=67.6 \mathrm{~Hz}), 30.0(\mathrm{~d}, J=2.3 \mathrm{~Hz}), 20.8 .{ }^{31} \mathrm{P}$ NMR (162 MHz, $\left.\mathrm{CDCl}_{3}\right) \delta$ 27.4. HRMS: m/z (EI) calculated $[\mathrm{M}]^{+}$345.1283, measured: 345.1273 .

${ }^{[\alpha]} \mathrm{D}^{29.3} 119.0643$ (c 1.0, $\mathrm{CHCl}_{3}$ ). HPLC (IC-3, 0.46*15 cm, $3 \mu \mathrm{m}$, hexane/ isopropanol $=6 / 4$, flow $0.7 \mathrm{~mL} / \mathrm{min}$, detection at $214 \mathrm{~nm}$ ) retention time $=14.940 \mathrm{~min}$ (major) and $19.668 \min$ (minor).

3f<smiles>N#C[C@@H](CP=O)c1ccc(CCl)cc1</smiles>

White solid. mp $145{ }^{\circ} \mathrm{C}-146{ }^{\circ} \mathrm{C}$.

${ }^{1} \mathrm{H}$ NMR $\left(400 \mathrm{MHz}, \mathrm{CDCl}_{3}\right) \delta 7.82-7.73(\mathrm{~m}, 2 \mathrm{H}), 7.67-7.44(\mathrm{~m}, 5 \mathrm{H}), 7.44-7.40$ (m, 1H), $7.40-7.27(\mathrm{~m}, 4 \mathrm{H}), 7.23(\mathrm{~d}, J=8.0 \mathrm{~Hz}, 2 \mathrm{H}), 4.62-4.32(\mathrm{~m}, 3 \mathrm{H}), 3.08-$ $2.96(\mathrm{~m}, 1 \mathrm{H}), 2.84-2.72(\mathrm{~m}, 1 \mathrm{H}) .{ }^{13} \mathrm{C} \mathrm{NMR}\left(100 \mathrm{MHz}, \mathrm{CDCl}_{3}\right) \delta 137.8,135.3(\mathrm{~d}, J$ $=6.9 \mathrm{~Hz}), 132.5(\mathrm{~d}, J=2.3 \mathrm{~Hz}), 132.1(\mathrm{~d}, J=3.0 \mathrm{~Hz}), 131.5(\mathrm{~d}, J=101.2 \mathrm{~Hz}), 131.5$ $(\mathrm{d}, J=101.2 \mathrm{~Hz}), 130.8(\mathrm{~d}, J=11.2 \mathrm{~Hz}), 130.5(\mathrm{~d}, J=9.2 \mathrm{~Hz}), 129.3,128.9(\mathrm{~d}, J=$ $11.4 \mathrm{~Hz}), 128.7$ (d, $J=12.3 \mathrm{~Hz}), 128.0,119.7$ (d, $J=9.9 \mathrm{~Hz}), 45.3,36.0(\mathrm{~d}, J=67.4$ $\mathrm{Hz}), 30.3(\mathrm{~d}, J=2.3 \mathrm{~Hz}) .{ }^{31} \mathrm{P}$ NMR $\left(162 \mathrm{MHz}, \mathrm{CDCl}_{3}\right) \delta 27.7 . \mathrm{HRMS}: \mathrm{m} / \mathrm{z}(\mathrm{EI})$ calculated $[\mathrm{M}]+379.0893$, measured: 379.0886 .

${ }^{[\alpha]} \mathrm{D}^{28.0} 79.4271$ (c 1.0, $\mathrm{CHCl}_{3}$ ). HPLC (IC-3, 0.46*15 cm, $3 \mu \mathrm{m}$, hexane/ isopropanol $=6 / 4$, flow $0.7 \mathrm{~mL} / \mathrm{min}$, detection at $214 \mathrm{~nm}$ ) retention time $=14.440 \mathrm{~min}$ (major) and $19.633 \mathrm{~min}$ (minor).

$3 g$<smiles>CC(C)(C)c1ccc(C(C#N)CPOc2ccccc2)cc1</smiles>

White solid. mp $150{ }^{\circ} \mathrm{C}-151^{\circ} \mathrm{C}$. 
${ }^{1} \mathrm{H}$ NMR (400 MHz, $\left.\mathrm{CDCl}_{3}\right) \delta 7.77(\mathrm{dd}, J=11.6,7.2 \mathrm{~Hz}, 2 \mathrm{H}), 7.59-7.47(\mathrm{~m}, 5 \mathrm{H})$, $7.42(\mathrm{t}, J=6.4 \mathrm{~Hz} 1 \mathrm{H}), 7.28-7.35(\mathrm{~m}, 2 \mathrm{H}), 7.25-7.16(\mathrm{~m}, 4 \mathrm{H}), 4.50-4.40(\mathrm{~m}, 1 \mathrm{H})$, $3.05-2.95(\mathrm{~m}, 1 \mathrm{H}), 2.85-2.74(\mathrm{~m}, 1 \mathrm{H}), 1.24(\mathrm{~s}, 9 \mathrm{H}) .{ }^{13} \mathrm{C} \mathrm{NMR}(100 \mathrm{MHz}, \mathrm{CDCl} 3)$ $\delta 151.4,132.3(\mathrm{~d}, J=3.2 \mathrm{~Hz}), 132.0(\mathrm{~d}, J=6.4 \mathrm{~Hz}), 131.8(\mathrm{~d}, J=2.8 \mathrm{~Hz}), 131.7(\mathrm{~d}, J$ = $100.0 \mathrm{~Hz}), 131.6(\mathrm{~d}, J=100.4 \mathrm{~Hz}), 130.8(\mathrm{~d}, J=9.7 \mathrm{~Hz}), 130.5(\mathrm{~d}, J=9.7 \mathrm{~Hz})$, $128.8(\mathrm{~d}, J=12.1 \mathrm{~Hz}), 128.5(\mathrm{~d}, J=12.1 \mathrm{~Hz}), 127.2,126.0,120.0(\mathrm{~d}, J=9.7 \mathrm{~Hz})$, $36.1(\mathrm{~d}, J=68.6 \mathrm{~Hz}), 34.4,31.1,30.0(\mathrm{~d}, J=2.0 \mathrm{~Hz}) .{ }^{31} \mathrm{P} \mathrm{NMR}\left(162 \mathrm{MHz}, \mathrm{CDCl}_{3}\right) \delta$ 28.4. HRMS: m/z (EI) calculated [M]+387.1752, measured: 387.1757 .

${ }^{[\alpha]} \mathrm{D}^{29.2} 234.3693\left(\mathrm{c} 0.4, \mathrm{CHCl}_{3}\right)$. HPLC (IC-3, 0.46*15 cm, $3 \mu \mathrm{m}$, hexane/ isopropanol $=6 / 4$, flow $0.7 \mathrm{~mL} / \mathrm{min}$, detection at $214 \mathrm{~nm}$ ) retention time $=11.367 \mathrm{~min}$ (major) and $13.442 \mathrm{~min}$ (minor).

$3 \mathbf{h}$<smiles>N#C[C@@H](CPOc1ccccc1)c1ccc(-c2ccccc2)cc1</smiles>

White solid. mp $154{ }^{\circ} \mathrm{C}-155^{\circ} \mathrm{C}$.

${ }^{1} \mathrm{H}$ NMR (400 MHz, $\left.\mathrm{CDCl}_{3}\right) \delta 7.83-7.73(\mathrm{~m}, 2 \mathrm{H}), 7.60-7.29(\mathrm{~m}, 17 \mathrm{H}), 4.65-4.32$ $(\mathrm{m}, 1 \mathrm{H}), 3.15-2.98(\mathrm{~m}, 1 \mathrm{H}), 2.97-2.75(\mathrm{~m}, 1 \mathrm{H}) .{ }^{13} \mathrm{C} \mathrm{NMR}\left(100 \mathrm{MHz}, \mathrm{CDCl}_{3}\right) \delta$ 141.3, 140.0, 133.9 (d, $J=6.0 \mathrm{~Hz}), 132.3$ (d, $J=2.2 \mathrm{~Hz}), 131.7$ (d, $J=3.0 \mathrm{~Hz}), 131.6$ (d, $J=101.3 \mathrm{~Hz}), 131.5$ (d, $J=101.2 \mathrm{~Hz}), 130.7$ (d, $J=9.7 \mathrm{~Hz}), 130.4$ (d, $J=9.6 \mathrm{~Hz})$, $128.8,128.7,128.5(\mathrm{~d}, J=11.9 \mathrm{~Hz}), 128.0,127.6,127.5,127.0,119.8$ (d, $J=10.4 \mathrm{~Hz})$, $36.1(\mathrm{~d}, J=67.7 \mathrm{~Hz}), 30.3(\mathrm{~d}, J=2.2 \mathrm{~Hz}) .{ }^{31} \mathrm{P}$ NMR $\left(162 \mathrm{MHz}, \mathrm{CDCl}_{3}\right) \delta$ 27.9. HRMS: m/z (ESI) calculated $[\mathrm{M}+\mathrm{H}]^{+}$408.1512, measured: 408.1512.

${ }^{[\alpha]} \mathrm{D}^{27.0} 22.6260\left(\mathrm{c} 4.0, \mathrm{CHCl}_{3}\right)$. HPLC (IC-3, 0.46*15 cm, $3 \mu \mathrm{m}$, hexane/ isopropanol $=6 / 4$, flow $0.7 \mathrm{~mL} / \mathrm{min}$, detection at $214 \mathrm{~nm}$ ) retention time $=15.560 \mathrm{~min}$ (major) and $19.195 \min$ (minor).

3i<smiles>CC(=O)Oc1ccc([C@@H](C#N)CPOc2ccccc2)cc1</smiles> 
White solid. mp $151{ }^{\circ} \mathrm{C}-152{ }^{\circ} \mathrm{C}$.

${ }^{1} \mathrm{H}$ NMR $\left(400 \mathrm{MHz}, \mathrm{CDCl}_{3}\right) \delta 7.83-7.73(\mathrm{~m}, 2 \mathrm{H}), 7.62-7.42(\mathrm{~m}, 6 \mathrm{H}), 7.41-7.34$ $(\mathrm{m}, 1 \mathrm{H}), 7.32(\mathrm{~d}, J=8.0,2 \mathrm{H}), 6.96(\mathrm{~d}, J=8.4 \mathrm{~Hz}, 2 \mathrm{H}), 4.62-4.32(\mathrm{~m}, 1 \mathrm{H}), 3.08-$ $2.95(\mathrm{~m}, 1 \mathrm{H}), 2.82-2.71(\mathrm{~m}, 1 \mathrm{H}), 2.27(\mathrm{~s}, 3 \mathrm{H}) .{ }^{13} \mathrm{C} \mathrm{NMR}\left(100 \mathrm{MHz}, \mathrm{CDCl}_{3}\right) \delta 169.0$, $150.5,132.8(\mathrm{~d}, J=6.9 \mathrm{~Hz}), 132.5(\mathrm{~d}, J=3.2 \mathrm{~Hz}), 132.1(\mathrm{~d}, J=2.9 \mathrm{~Hz}), 131.5(\mathrm{~d}, J=$ $100.5 \mathrm{~Hz}), 131.3(\mathrm{~d}, J=100.0 \mathrm{~Hz}), 130.9$ (d, $J=9.7 \mathrm{~Hz}), 130.5(\mathrm{~d}, J=9.3 \mathrm{~Hz}), 128.9$ $(\mathrm{d}, J=12.1 \mathrm{~Hz}), 128.8,128.6,122.3,119.6(\mathrm{~d}, J=8.9 \mathrm{~Hz}), 36.3(\mathrm{~d}, J=67.8 \mathrm{~Hz}), 30.0$ $(\mathrm{d}, J=2.4 \mathrm{~Hz}), 21.0 .{ }^{31} \mathrm{P}$ NMR $\left(162 \mathrm{MHz}, \mathrm{CDCl}_{3}\right) \delta 27.4$. HRMS: $\mathrm{m} / \mathrm{z}$ (EI) calculated $[\mathrm{M}]^{+}$389.1181, measured: 389.1177 .

${ }^{[\alpha]} \mathrm{D}^{28.6} 111.2287$ (c 1.0, $\left.\mathrm{CHCl}_{3}\right)$. HPLC (IC-3, 0.46*15 cm, $3 \mu \mathrm{m}$, hexane/ isopropanol $=6 / 4$, flow $0.7 \mathrm{~mL} / \mathrm{min}$, detection at $214 \mathrm{~nm}$ ) retention time $=18.850 \mathrm{~min}$ (major) and $25.478 \mathrm{~min}$ (minor).

3j<smiles>N#C[C@@H](CPO)c1ccc(OP)cc1</smiles>

White solid. mp $152{ }^{\circ} \mathrm{C}-153{ }^{\circ} \mathrm{C}$.

${ }^{1} \mathrm{H}$ NMR (400 MHz, $\left.\mathrm{CDCl}_{3}\right) \delta 7.81-7.74(\mathrm{~m}, 2 \mathrm{H}), 7.65-7.55(\mathrm{~m}, 3 \mathrm{H}), 7.54-7.46$ (m, 3H), $7.41-7.32(\mathrm{~m}, 4 \mathrm{H}), 7.28-7.24(\mathrm{~m}, 2 \mathrm{H}), 7.16-7.10(\mathrm{~m}, 1 \mathrm{H}), 6.94(\mathrm{~d}, J=$ $7.6 \mathrm{~Hz}, 2 \mathrm{H}), 6.82(\mathrm{~d}, J=8.8 \mathrm{~Hz}, 2 \mathrm{H}), 4.55-4.40(\mathrm{~m}, 1 \mathrm{H}), 3.09-2.97$ (m, 1H), 2.87 $-2.76(\mathrm{~m}, 1 \mathrm{H}) .{ }^{13} \mathrm{C} \mathrm{NMR}\left(100 \mathrm{MHz}, \mathrm{CDCl}_{3}\right) \delta 157.5,156.4,132.5(\mathrm{~d}, J=3.0 \mathrm{~Hz})$, $131.9(\mathrm{~d}, J=2.2 \mathrm{~Hz}), 131.6(\mathrm{~d}, J=99.7 \mathrm{~Hz}), 131.5(\mathrm{~d}, J=102.0 \mathrm{~Hz}), 130.8(\mathrm{~d}, J=$ $9.7 \mathrm{~Hz}), 130.5(\mathrm{~d}, J=9.7 \mathrm{~Hz}), 129.8,129.5(\mathrm{~d}, J=6.0 \mathrm{~Hz}), 129.1,128.9(\mathrm{~d}, J=11.3$ $\mathrm{Hz}), 128.7$ (d, $J=11.9 \mathrm{~Hz}), 123.8,120.0$ (d, $J=10.5 \mathrm{~Hz}), 119.2,118.9,36.1$ (d, $J=$ $67.7 \mathrm{~Hz}), 30.0(\mathrm{~d}, J=2.2 \mathrm{~Hz}) .{ }^{31} \mathrm{P}$ NMR $\left(162 \mathrm{MHz}, \mathrm{CDCl}_{3}\right) \delta 28.0 . \mathrm{HRMS}: \mathrm{m} / \mathrm{z}(\mathrm{EI})$ calculated $[\mathrm{M}]^{+}$423.1388, measured: 423.1385 .

${ }^{[\alpha]} \mathrm{D}^{29.2} 245.0993\left(\mathrm{c} 1.0, \mathrm{CHCl}_{3}\right)$. HPLC (IF-3, 0.46*15 cm, $3 \mu \mathrm{m}$, hexane/ isopropanol $=6 / 4$, flow $0.7 \mathrm{~mL} / \mathrm{min}$, detection at $214 \mathrm{~nm}$ ) retention time $=26.46 \mathrm{~min}$ (major) and 30.35 min (minor). 
MeOOC

White solid. mp $148{ }^{\circ} \mathrm{C}-149^{\circ} \mathrm{C}$.

${ }^{1} \mathrm{H}$ NMR $\left(400 \mathrm{MHz}, \mathrm{CDCl}_{3}\right) \delta 7.88(\mathrm{~d}, J=8.0 \mathrm{~Hz}, 2 \mathrm{H}), 7.83-7.73(\mathrm{~m}, 2 \mathrm{H}), 7.61-$ $7.49(\mathrm{~m}, 5 \mathrm{H}), 7.47-7.32(\mathrm{~m}, 5 \mathrm{H}), 4.58-4.48(\mathrm{~m}, 1 \mathrm{H}), 3.90$ (s, 3H), $3.10-3.00(\mathrm{~m}$, 1H), $2.82-2.732(\mathrm{~m}, 1 \mathrm{H}) .{ }^{13} \mathrm{C}$ NMR $\left(100 \mathrm{MHz}, \mathrm{CDCl}_{3}\right) \delta 166.1,139.9(\mathrm{~d}, J=6.8 \mathrm{~Hz})$, $132.5(\mathrm{~d}, J=3.0 \mathrm{~Hz}), 132.0(\mathrm{~d}, J=3.0 \mathrm{~Hz}), 131.5(\mathrm{~d}, J=101.0 \mathrm{~Hz}), 131.3(\mathrm{~d}, J=$ $101.0 \mathrm{~Hz}), 130.9$ (d, $J=9.9 \mathrm{~Hz}), 130.4(\mathrm{~d}, J=9.1 \mathrm{~Hz}), 130.3,130.3,128.9$ (d, $J=$ 12.2 Hz), $128.7(\mathrm{~d}, J=12.1 \mathrm{~Hz}), 127.7,119.3(\mathrm{~d}, J=9.8 \mathrm{~Hz}), 52.3,35.9(\mathrm{~d}, J=67.6$ $\mathrm{Hz}), 30.5(\mathrm{~d}, J=2.3 \mathrm{~Hz}) .{ }^{31} \mathrm{P}$ NMR (162 MHz, $\left.\mathrm{CDCl}_{3}\right) \delta$ 27.0. HRMS: m/z (EI) calculated $[\mathrm{M}]^{+}$389.1181, measured: 389.1179 .

${ }^{[\alpha]} \mathrm{D}^{28.9} 184.5062\left(\mathrm{c} 0.5, \mathrm{CHCl}_{3}\right)$. HPLC (IC-3, 0.46*15 cm, $3 \mu \mathrm{m}$, hexane/ isopropanol $=6 / 4$, flow $0.7 \mathrm{~mL} / \mathrm{min}$, detection at $214 \mathrm{~nm}$ ) retention time $=20.250 \mathrm{~min}$ (major) and $30.695 \min$ (minor).

31<smiles>N#C[C@@H](CPO)c1ccc(C(F)(F)F)cc1</smiles>

White solid. mp $139{ }^{\circ} \mathrm{C}-140{ }^{\circ} \mathrm{C}$.

${ }^{1} \mathrm{H}$ NMR (400 MHz, $\left.\mathrm{CDCl}_{3}\right) \delta 7.79-7.71(\mathrm{~m}, 2 \mathrm{H}), 7.58-7.53(\mathrm{~m}, 1 \mathrm{H}), 7.53-7.46$ (m, 4H), $7.45-7.36(\mathrm{~m}, 5 \mathrm{H}), 7.33-7.26(\mathrm{~m}, 2 \mathrm{H}), 4.60-4.48(\mathrm{~m}, 1 \mathrm{H}), 3.10-3.00$ $(\mathrm{m}, 1 \mathrm{H}), 2.85-2.75(\mathrm{~m}, 1 \mathrm{H}) .{ }^{13} \mathrm{C} \mathrm{NMR}\left(100 \mathrm{MHz}, \mathrm{CDCl}_{3}\right) \delta 138.7(\mathrm{~d}, J=4.4 \mathrm{~Hz})$, $132.5(\mathrm{~d}, J=2.3 \mathrm{~Hz}), 132.0(\mathrm{~d}, J=3.1 \mathrm{~Hz}), 131.5(\mathrm{~d}, J=115.0 \mathrm{~Hz}), 131.2(\mathrm{~d}, J=$ 114.1 Hz), 131.1, 130.7 (d, $J=9.1 \mathrm{~Hz}), 130.4$ (d, $J=9.2 \mathrm{~Hz}), 128.9$ (d, $J=12.2 \mathrm{~Hz})$, $128.6(\mathrm{~d}, J=12.2 \mathrm{~Hz}), 128.3,125.9(\mathrm{q}, J=3.0 \mathrm{~Hz}), 123.5(\mathrm{q}, J=270.5 \mathrm{~Hz}), 119.5(\mathrm{~d}$, $J=11.5 \mathrm{~Hz}), 35.8(\mathrm{~d}, J=67.5 \mathrm{~Hz}), 30.5(\mathrm{~d}, J=1.5 \mathrm{~Hz}) .{ }^{31} \mathrm{P} \mathrm{NMR}\left(162 \mathrm{MHz}, \mathrm{CDCl}_{3}\right)$ $\delta$ 27.5. HRMS: m/z (EI) calculated [M] 399.1000, measured: 399.1004.

${ }^{[\alpha]} \mathrm{D}^{29.1} 99.9828$ (c 1.0, $\mathrm{CHCl}_{3}$ ). HPLC (IF-3, 0.46*15 cm, $3 \mu \mathrm{m}$, hexane/ isopropanol 
$=6 / 4$, flow $0.7 \mathrm{~mL} / \mathrm{min}$, detection at $214 \mathrm{~nm}$ ) retention time $=15.64$ min (major) and 19.41 min (minor).

$3 \mathbf{m}$<smiles>N#C[C@@H](CPO)c1cccc(Br)c1</smiles>

White solid. mp $147{ }^{\circ} \mathrm{C}-148{ }^{\circ} \mathrm{C}$.

${ }^{1} \mathrm{H}$ NMR (400 MHz, $\left.\mathrm{CDCl}_{3}\right) \delta 7.83-7.72(\mathrm{~m}, 2 \mathrm{H}), 7.66-7.41(\mathrm{~m}, 7 \mathrm{H}), 7.41-7.33$ (m, 2H), $7.33-7.24(\mathrm{~m}, 2 \mathrm{H}), 7.07(\mathrm{t}, J=8.0 \mathrm{~Hz}, 1 \mathrm{H}), 4.56-4.37(\mathrm{~m}, 1 \mathrm{H}), 3.09-$ $2.95(\mathrm{~m}, 1 \mathrm{H}), 2.86-2.73(\mathrm{~m}, 1 \mathrm{H}) .{ }^{13} \mathrm{C} \mathrm{NMR}\left(100 \mathrm{MHz}, \mathrm{CDCl}_{3}\right) \delta 137.0(\mathrm{~d}, J=6.0$ Hz), 132.6 (d, $J=2.3 \mathrm{~Hz}), 132.2$ (d, $J=3.0 \mathrm{~Hz}), 131.7,131.3$ (d, $J=101.9 \mathrm{~Hz}), 131.2$ $(\mathrm{d}, J=101.9 \mathrm{~Hz}), 130.9(\mathrm{~d}, J=9.1 \mathrm{~Hz}), 130.8,130.6(\mathrm{~d}, J=11.1 \mathrm{~Hz}), 130.4,129.0(\mathrm{~d}$, $J=11.9 \mathrm{~Hz}), 128.7(\mathrm{~d}, J=11.9 \mathrm{~Hz}), 126.4,123.0,119.4(\mathrm{~d}, J=10.7 \mathrm{~Hz}), 35.9(\mathrm{~d}, J=$ $67.2 \mathrm{~Hz}), 30.2(\mathrm{~d}, J=2.3 \mathrm{~Hz}) .{ }^{31} \mathrm{P} \mathrm{NMR}\left(162 \mathrm{MHz}, \mathrm{CDCl}_{3}\right) \delta 28.2 . \mathrm{HRMS}: \mathrm{m} / \mathrm{z}(\mathrm{ESI})$ calculated $[\mathrm{M}+\mathrm{H}]^{+}$410.0304, measured: 410.0300 .

${ }^{[\alpha]} \mathrm{D}^{29.2} 113.5180\left(\mathrm{c} 1.0, \mathrm{CHCl}_{3}\right)$. HPLC (IC-3, 0.46*15 cm, $3 \mu \mathrm{m}$, hexane/ isopropanol $=6 / 4$, flow $0.7 \mathrm{~mL} / \mathrm{min}$, detection at $214 \mathrm{~nm}$ ) retention time $=9.940 \mathrm{~min}$ (major) and $13.418 \min$ (minor).

\section{$3 n$}<smiles>Cc1cccc(C(C#N)CPO)c1</smiles>

White solid. mp $137^{\circ} \mathrm{C}-138^{\circ} \mathrm{C}$.

${ }^{1} \mathrm{H}$ NMR (400 MHz, $\left.\mathrm{CDCl}_{3}\right) \delta 7.81-7.74(\mathrm{~m}, 2 \mathrm{H}), 7.61-7.53(\mathrm{~m}, 3 \mathrm{H}), 7.53-7.47$ (m, 2H), $7.46-7.40(\mathrm{~m}, 1 \mathrm{H}), 7.38-7.31(\mathrm{~m}, 2 \mathrm{H}), 7.15-7.08(\mathrm{~m}, 2 \mathrm{H}), 7.07(\mathrm{~s}, 1 \mathrm{H})$, $7.02-6.95(\mathrm{~m}, 1 \mathrm{H}), 4.45-4.35(\mathrm{~m}, 1 \mathrm{H}), 3.06-2.95(\mathrm{~m}, 1 \mathrm{H}), 2.81-2.71(\mathrm{~m}, 1 \mathrm{H})$, $2.22(\mathrm{~s}, 3 \mathrm{H}),{ }^{13} \mathrm{C}$ NMR $\left(100 \mathrm{MHz}, \mathrm{CDCl}_{3}\right) \delta 138.9,135.1(\mathrm{~d}, J=6.9 \mathrm{~Hz}), 132.3(\mathrm{~d}, J$ $=2.3 \mathrm{~Hz}), 131.8(\mathrm{~d}, J=2.3 \mathrm{~Hz}), 131.8(\mathrm{~d}, J=101.1 \mathrm{~Hz}), 131.6(\mathrm{~d}, J=100.3 \mathrm{~Hz})$, $130.8(\mathrm{~d}, J=9.9 \mathrm{~Hz}), 130.4(\mathrm{~d}, J=9.2 \mathrm{~Hz}), 129.1(\mathrm{~d}, J=10.8 \mathrm{~Hz}), 128.8(\mathrm{~d}, J=11.5$ $\mathrm{Hz}), 128.5(\mathrm{~d}, J=12.3 \mathrm{~Hz}), 128.2,124.4,119.4(\mathrm{~d}, J=9.2 \mathrm{~Hz}), 36.1(\mathrm{~d}, J=67.4 \mathrm{~Hz})$, 
$30.4(\mathrm{~d}, J=2.3 \mathrm{~Hz}), 21.1 .{ }^{31} \mathrm{P}$ NMR $\left(162 \mathrm{MHz}, \mathrm{CDCl}_{3}\right) \delta 27.8 \mathrm{HRMS}: \mathrm{m} / \mathrm{z}(\mathrm{EI})$ calculated $[\mathrm{M}]^{+}$345.1283, measured: 345.1280.

${ }^{[\alpha]} \mathrm{D}^{22.8} 36.9271$ (c $0.5, \mathrm{CHCl}_{3}$ ). HPLC (IC-3, 0.46*15 cm, $3 \mu \mathrm{m}$, hexane/ isopropanol $=6 / 4$, flow $0.7 \mathrm{~mL} / \mathrm{min}$, detection at $214 \mathrm{~nm}$ ) retention time $=12.782 \mathrm{~min}$ (major) and $16.095 \min$ (minor).

30<smiles>COc1cccc(C(C#N)CPOc2ccccc2)c1</smiles>

White solid. mp $92{ }^{\circ} \mathrm{C}-94{ }^{\circ} \mathrm{C}$.

${ }^{1} \mathrm{H}$ NMR (400 MHz, $\left.\mathrm{CDCl}_{3}\right) \delta 7.82-7.76(\mathrm{~m}, 2 \mathrm{H}), 7.62-7.55(\mathrm{~m}, 3 \mathrm{H}), 7.54-7.49$ (m, 2H), $7.48-7.43(\mathrm{~m}, 1 \mathrm{H}) .7 .40-7.33(\mathrm{~m}, 2 \mathrm{H}), 7.16(\mathrm{t}, J=8.0 \mathrm{~Hz}, 1 \mathrm{H}), 6.91$ (d, $J$ $=7.6 \mathrm{~Hz}, 1 \mathrm{H}), 6.81(\mathrm{~s}, 1 \mathrm{H}), 6.77-6.67(\mathrm{~m}, 1 \mathrm{H}), 4.49-4.37(\mathrm{~m}, 1 \mathrm{H}), 3.73(\mathrm{~s}, 3 \mathrm{H})$. $3.06-2.97(\mathrm{~m}, 1 \mathrm{H}), 2.83-2.74(\mathrm{~m}, 1 \mathrm{H}) .{ }^{13} \mathrm{C} \mathrm{NMR}\left(100 \mathrm{MHz}, \mathrm{CDCl}_{3}\right) \delta 159.9,136.7$ $(\mathrm{d}, J=7.5 \mathrm{~Hz}), 132.5$ (d, $J=3.0 \mathrm{~Hz}), 132.0(\mathrm{~d}, J=3.0 \mathrm{~Hz}), 131.8(\mathrm{~d}, J=101.2 \mathrm{~Hz})$, $131.7(\mathrm{~d}, J=101.2 \mathrm{~Hz}), 130.9(\mathrm{~d}, J=9.0 \mathrm{~Hz}), 130.5(\mathrm{~d}, J=9.7 \mathrm{~Hz}), 130.3,128.9$ (d, $J$ = 11.9 Hz), $128.6(\mathrm{~d}, J=12.1 \mathrm{~Hz}), 119.7(\mathrm{~d}, J=8.9 \mathrm{~Hz}), 119.6,114.2,113.0,55.3$, $36.2(\mathrm{~d}, J=67.0 \mathrm{~Hz}), 30.5(\mathrm{~d}, J=3.0 \mathrm{~Hz}) .{ }^{31} \mathrm{P} \mathrm{NMR}\left(162 \mathrm{MHz}, \mathrm{CDCl}_{3}\right) \delta 27.9 \mathrm{HRMS}$ : $\mathrm{m} / \mathrm{z}$ (EI) calculated [M] $]^{+}$361.1232, measured: 361.1237.

${ }^{[\alpha]} \mathrm{D}^{23.2}-13.7105\left(\mathrm{c} 0.5, \mathrm{CHCl}_{3}\right)$. HPLC (IC-3, 0.46*15 cm, $3 \mu \mathrm{m}$, hexane/ isopropanol $=6 / 4$, flow $0.7 \mathrm{~mL} / \mathrm{min}$, detection at $214 \mathrm{~nm}$ ) retention time $=14.837 \mathrm{~min}$ (major) and $18.295 \min$ (minor).

$3 \mathbf{p}$<smiles>COc1cc(OC)cc([C@H](C#N)CPOc2ccccc2)c1</smiles>

White solid. mp $95{ }^{\circ} \mathrm{C}-96{ }^{\circ} \mathrm{C}$.

${ }^{1} \mathrm{H}$ NMR (400 MHz, $\left.\mathrm{CDCl}_{3}\right) \delta 7.83-7.75(\mathrm{~m}, 2 \mathrm{H}), 7.64-7.55(\mathrm{~m}, 3 \mathrm{H}), 7.54-7.43$ (m, 3H), $7.41-7.34(\mathrm{~m}, 2 \mathrm{H}) .6 .44(\mathrm{~d}, J=2.0 \mathrm{~Hz}, 2 \mathrm{H}), 6.26(\mathrm{t}, J=2.0 \mathrm{~Hz}, 1 \mathrm{H}), 4.44-$ 
$4.34(\mathrm{~m}, 1 \mathrm{H}), 3.72(\mathrm{~s}, 6 \mathrm{H}) .3 .06-2.94(\mathrm{~m}, 1 \mathrm{H}), 2.85-2.72(\mathrm{~m}, 1 \mathrm{H}) .{ }^{13} \mathrm{C} \mathrm{NMR}(100$ $\left.\mathrm{MHz}, \mathrm{CDCl}_{3}\right) \delta 161.2,137.4(\mathrm{~d}, J=6.7 \mathrm{~Hz}), 132.5(\mathrm{~d}, J=2.3 \mathrm{~Hz}), 132.0(\mathrm{~d}, J=2.6$ Hz), 130.9 (d, $J=9.7 \mathrm{~Hz}), 130.5$ (d, $J=9.7 \mathrm{~Hz}), 128.9$ (d, $J=11.9 \mathrm{~Hz}), 128.6$ (d, $J=$ $12.6 \mathrm{~Hz}), 119.7(\mathrm{~d}, J=9.0 \mathrm{~Hz}), 105.5,100.5,55.4,36.1$ (d, $J=67.8 \mathrm{~Hz}), 30.7$ (d, $J=$ $2.3 \mathrm{~Hz}) .{ }^{31} \mathrm{P}$ NMR $\left(162 \mathrm{MHz}, \mathrm{CDCl}_{3}\right) \delta 28.0 \mathrm{HRMS}: \mathrm{m} / \mathrm{z}$ (EI) calculated $[\mathrm{M}]^{+}$ 391.1337, measured: 391.1331.

${ }^{[\alpha]} \mathrm{D}^{28.5} 80.0977$ (c 1.0, $\mathrm{CHCl}_{3}$ ). HPLC (IC-3, 0.46*15 cm, $3 \mu \mathrm{m}$, hexane/ isopropanol $=6 / 4$, flow $0.7 \mathrm{~mL} / \mathrm{min}$, detection at $214 \mathrm{~nm}$ ) retention time $=16.473 \mathrm{~min}$ (major) and $19.283 \min (\operatorname{minor})$.

$3 \mathbf{q}$<smiles>N#C[C@@H](CPO)c1ccccc1Cl</smiles>

White solid. mp $121{ }^{\circ} \mathrm{C}-122{ }^{\circ} \mathrm{C}$.

${ }^{1} \mathrm{H}$ NMR $\left(400 \mathrm{MHz}, \mathrm{CDCl}_{3}\right) \delta 7.83-7.75(\mathrm{~m}, 2 \mathrm{H}), 7.70-7.63(\mathrm{~m}, 2 \mathrm{H}), 7.69-7.53$ (m, 1H). $7.53-7.43(\mathrm{~m}, 4 \mathrm{H}), 7.42-7.34(\mathrm{~m}, 2 \mathrm{H}), 7.26-7.13(\mathrm{~m}, 3 \mathrm{H}), 4.83-4.67$ $(\mathrm{m}, 1 \mathrm{H}), 3.00-2.86(\mathrm{~m}, 2 \mathrm{H}) .{ }^{13} \mathrm{C} \mathrm{NMR}\left(100 \mathrm{MHz}, \mathrm{CDCl}_{3}\right) \delta 132.7,132.6,132.3(\mathrm{~d}, J$ = 3.1 Hz), $132.0(\mathrm{~d}, J=3.0 \mathrm{~Hz}), 131.0(\mathrm{~d}, J=3.9 \mathrm{~Hz}), 130.8(\mathrm{~d}, J=10.0 \mathrm{~Hz}), 130.6$ $(\mathrm{d}, J=9.2 \mathrm{~Hz}), 130.2,130.0(\mathrm{~d}, J=3.1 \mathrm{~Hz}), 130.3,128.2(\mathrm{~d}, J=12.2 \mathrm{~Hz}), 128.6(\mathrm{~d}, J$ $=11.5 \mathrm{~Hz}), 127.6,118.6(\mathrm{~d}, J=6.9 \mathrm{~Hz}), 33.6(\mathrm{~d}, J=67.4 \mathrm{~Hz}), 28.9(\mathrm{~d}, J=2.2 \mathrm{~Hz})$. ${ }^{31} \mathrm{P}$ NMR $\left(162 \mathrm{MHz}, \mathrm{CDCl}_{3}\right) \delta 27.6 \mathrm{HRMS}: \mathrm{m} / \mathrm{z}(\mathrm{EI})$ calculated $[\mathrm{M}]^{+}$365.0736, measured: 365.0728 .

${ }^{[\alpha]} \mathrm{D}^{28.2} 118.8884$ (c 1.0, $\mathrm{CHCl}_{3}$ ). HPLC (IC-3, 0.46*15 cm, $3 \mu \mathrm{m}$, hexane/ isopropanol $=6 / 4$, flow $0.7 \mathrm{~mL} / \mathrm{min}$, detection at $214 \mathrm{~nm}$ ) retention time $=14.670 \mathrm{~min}$ (major) and $21.205 \min$ (minor).

$3 \mathbf{r}$<smiles>N#C[C@@H](CPO)c1ccc2ccccc2c1</smiles>

White solid. mp $141{ }^{\circ} \mathrm{C}-142{ }^{\circ} \mathrm{C}$. 
${ }^{1} \mathrm{H}$ NMR (400 MHz, $\left.\mathrm{CDCl}_{3}\right) \delta 7.83-7.77(\mathrm{~m}, 2 \mathrm{H}), 7.76-7.68(\mathrm{~m}, 4 \mathrm{H}), 7.60-7.55$ (m, 1H), $7.54-7.44(\mathrm{~m}, 6 \mathrm{H}), 7.41-7.37(\mathrm{~m}, 1 \mathrm{H}), 7.23(\mathrm{~d}, J=2.3 \mathrm{~Hz}, 1 \mathrm{H}), 7.21-$ $7.08(\mathrm{~m}, 2 \mathrm{H}), 4.75-4.55(\mathrm{~m}, 1 \mathrm{H}), 3.17-3.04(\mathrm{~m}, 1 \mathrm{H}), 2.94-2.81(\mathrm{~m}, 1 \mathrm{H}) .{ }^{13} \mathrm{C}$ NMR (100 MHz, $\left.\mathrm{CDCl}_{3}\right) \delta 133.0,132.8,132.4(\mathrm{~d}, J=2.8 \mathrm{~Hz}), 132.2(\mathrm{~d}, J=6.5 \mathrm{~Hz})$, $131.6(\mathrm{~d}, J=2.8 \mathrm{~Hz}), 131.6(\mathrm{~d}, J=100.5 \mathrm{~Hz}), 131.5(\mathrm{~d}, J=100.9 \mathrm{~Hz}), 130.8$ (d, $J=$ $9.3 \mathrm{~Hz}), 130.3(\mathrm{~d}, J=9.3 \mathrm{~Hz}), 129.3,128.9$ (d, $J=12.1 \mathrm{~Hz}), 128.3(\mathrm{~d}, J=12.1 \mathrm{~Hz})$, 127.9, 127.5, 127.2, 126.6, 124.5, 119.9 (d, $J=9.3 \mathrm{~Hz}), 36.2$ (d, $J=67.8 \mathrm{~Hz}), 30.7$ (d, $J=2.0 \mathrm{~Hz}) .{ }^{31} \mathrm{P}$ NMR $\left(162 \mathrm{MHz}, \mathrm{CDCl}_{3}\right) \delta 27.7$. HRMS: m/z (EI) calculated $[\mathrm{M}]^{+}$ 381.1283, measured: 381.1284.

${ }^{[\alpha]} \mathrm{D}^{29.1} 157.5727\left(\mathrm{c} 1.0, \mathrm{CHCl}_{3}\right)$. HPLC (IC-3, 0.46*15 cm, $3 \mu \mathrm{m}$, hexane/ isopropanol $=6 / 4$, flow $0.7 \mathrm{~mL} / \mathrm{min}$, detection at $214 \mathrm{~nm}$ ) retention time $=14.462 \mathrm{~min}$ (major) and $18.833 \min$ (minor).

3s<smiles>N#C[C@H](CPOc1ccccc1)c1ccc2cc(Br)ccc2c1</smiles>

White solid. mp $142{ }^{\circ} \mathrm{C}-143{ }^{\circ} \mathrm{C}$.

${ }^{1} \mathrm{H}$ NMR (400 MHz, $\left.\mathrm{CDCl}_{3}\right) \delta 7.90(\mathrm{~s}, 1 \mathrm{H}), 7.81-7.74(\mathrm{~m}, 2 \mathrm{H}), 7.71(\mathrm{~s}, 1 \mathrm{H}), 7.63-$ $7.55(\mathrm{~m}, 3 \mathrm{H}), 7.55-7.44(\mathrm{~m}, 5 \mathrm{H}), 7.44-7.39(\mathrm{~m}, 1 \mathrm{H}), 7.26-7.21(\mathrm{~m}, 1 \mathrm{H}), 7.21-$ $7.06(\mathrm{~m}, 2 \mathrm{H}), 4.68-4.59(\mathrm{~m}, 1 \mathrm{H}), 3.14-3.04(\mathrm{~m}, 1 \mathrm{H}), 2.91-2.81(\mathrm{~m}, 1 \mathrm{H}) .{ }^{13} \mathrm{C}$ NMR (100 MHz, $\left.\mathrm{CDCl}_{3}\right) \delta 137.0(\mathrm{~d}, J=6.8 \mathrm{~Hz}), 132.5(\mathrm{~d}, J=3.0 \mathrm{~Hz}), 132.0(\mathrm{~d}, J=$ $3.0 \mathrm{~Hz}), 131.6,131.4(\mathrm{~d}, J=100.9 \mathrm{~Hz}), 131.2(\mathrm{~d}, J=100.9 \mathrm{~Hz}), 130.7(\mathrm{~d}, J=9.9 \mathrm{~Hz})$, 130.7, 130.6, $130.4(\mathrm{~d}, J=9.9 \mathrm{~Hz}), 128.9$ (d, $J=12.1 \mathrm{~Hz}), 128.6(\mathrm{~d}, J=12.1 \mathrm{~Hz})$, 126.2, 122.9, $119.3(\mathrm{~d}, J=10.7 \mathrm{~Hz}), 35.8(\mathrm{~d}, J=67.5 \mathrm{~Hz}), 30.2(\mathrm{~d}, J=2.3 \mathrm{~Hz}) .{ }^{31} \mathrm{P}$ NMR (162 MHz, $\left.\mathrm{CDCl}_{3}\right) \delta$ 27.6. HRMS: m/z (EI) calculated [M] ${ }^{+}$459.0388, measured: 459.0375 .

${ }^{[\alpha]} \mathrm{D}^{22.5} 82.6092$ (c $0.5, \mathrm{CHCl}_{3}$ ). HPLC (IF-3, 0.46*15 cm, $3 \mu \mathrm{m}$, hexane/ isopropanol $=6 / 4$, flow $0.7 \mathrm{~mL} / \mathrm{min}$, detection at $214 \mathrm{~nm}$ ) retention time $=21.21 \mathrm{~min}$ (major) and $24.18 \min$ (minor). 
<smiles>CC(=O)Oc1ccc2cc(C(C#N)CPOc3ccccc3)ccc2c1</smiles>

White solid. mp $145{ }^{\circ} \mathrm{C}-146{ }^{\circ} \mathrm{C}$.

${ }^{1} \mathrm{H}$ NMR (400 MHz, $\left.\mathrm{CDCl}_{3}\right) \delta 7.81-7.74(\mathrm{~m}, 2 \mathrm{H}), 7.74(\mathrm{~s}, 1 \mathrm{H}), 7.70(\mathrm{~d}, J=8.8 \mathrm{~Hz}$, 1H), $7.65(\mathrm{~d}, J=8.4 \mathrm{~Hz}, 1 \mathrm{H}), 7.59-7.53(\mathrm{~m}, 1 \mathrm{H}), 7.52-7.44(\mathrm{~m}, 5 \mathrm{H}), 7.40(\mathrm{~d}, J=$ $8.4 \mathrm{~Hz}, 1 \mathrm{H}), 7.26-7.20(\mathrm{~m}, 2 \mathrm{H}), 7.19-7.13(\mathrm{~m}, 2 \mathrm{H}), 4.70-4.55$ (m, 1H), $3.16-$ $3.03(\mathrm{~m}, 1 \mathrm{H}), 2.92-2.80(\mathrm{~m}, 1 \mathrm{H}), 2.35(\mathrm{~s}, 3 \mathrm{H}) .{ }^{13} \mathrm{C} \mathrm{NMR}\left(100 \mathrm{MHz}, \mathrm{CDCl}_{3}\right) \delta 169.4$, 149.0, 133.2, 132.4 (d, $J=1.6 \mathrm{~Hz}), 132.0$ (d, $J=5.7 \mathrm{~Hz}), 131.7$ (d, $J=1.2 \mathrm{~Hz}), 131.5$ (d, $J=99.3 \mathrm{~Hz}), 131.4$ (d, $J=99.2 \mathrm{~Hz}), 130.9,130.8$ (d, $J=9.7 \mathrm{~Hz}), 130.3$ (d, $J=9.3$ $\mathrm{Hz}), 129.3,129.0,128.9(\mathrm{~d}, J=12.5 \mathrm{~Hz}), 128.3(\mathrm{~d}, J=12.2 \mathrm{~Hz}), 127.2,125.2,122.0$, $119.8(\mathrm{~d}, J=10.0 \mathrm{~Hz}), 118.3,36.0(\mathrm{~d}, J=68.1 \mathrm{~Hz}), 30.7(\mathrm{~d}, J=1.6 \mathrm{~Hz}), 21.1 .{ }^{31} \mathrm{P}$ NMR $\left(162 \mathrm{MHz}, \mathrm{CDCl}_{3}\right) \delta 27.4 \mathrm{HRMS}: \mathrm{m} / \mathrm{z}(\mathrm{EI})$ calculated $[\mathrm{M}+\mathrm{H}]^{+}$440.1410, measured: 440.1411 .

${ }^{[\alpha]} \mathrm{D}^{23.9} 97.5762\left(\mathrm{c} 0.5, \mathrm{CHCl}_{3}\right)$. HPLC (IF-3, 0.46*15 cm, $3 \mu \mathrm{m}$, hexane/ isopropanol $=6 / 4$, flow $0.7 \mathrm{~mL} / \mathrm{min}$, detection at $214 \mathrm{~nm}$ ) retention time $=42.03 \mathrm{~min}$ (minor) and $48.95 \min$ (major).

$3 \mathbf{u}$<smiles>COc1ccc([C@@H](C#N)CPOc2ccccc2)cn1</smiles>

White solid. mp $138{ }^{\circ} \mathrm{C}-139{ }^{\circ} \mathrm{C}$.

${ }^{1} \mathrm{H}$ NMR $\left(400 \mathrm{MHz}, \mathrm{CDCl}_{3}\right) \delta 8.06(\mathrm{~d}, J=2.4 \mathrm{~Hz}, 1 \mathrm{H}), 7.79-7.73(\mathrm{~m}, 2 \mathrm{H}), 7.60-$ $7.42(\mathrm{~m}, 7 \mathrm{H}), 7.40-7.30(\mathrm{~m}, 2 \mathrm{H}), 6.53(\mathrm{~d}, J=8.8 \mathrm{~Hz}, 1 \mathrm{H}), 4.50-4.40(\mathrm{~m}, 1 \mathrm{H}), 3.86$ (s, 3H), $3.16-3.03(\mathrm{~m}, 1 \mathrm{H}), 3.08-2.99(\mathrm{~m}, 1 \mathrm{H}) .{ }^{13} \mathrm{C} \mathrm{NMR}\left(100 \mathrm{MHz}, \mathrm{CDCl}_{3}\right) \delta$ 164.0, 146.3, 137.6, 132.5 (d, $J=1.7 \mathrm{~Hz}), 131.9$ (d, $J=2.8 \mathrm{~Hz}), 131.6(\mathrm{~d}, J=101.7$ Hz), 131.5 (d, $J=101.7 \mathrm{~Hz}), 130.7$ (d, $J=9.5 \mathrm{~Hz}), 130.5$ (d, $J=9.0 \mathrm{~Hz}), 129.0$ (d, $J$ $=12.7 \mathrm{~Hz}), 128.6(\mathrm{~d}, J=11.7 \mathrm{~Hz}), 119.6(\mathrm{~d}, J=9.0 \mathrm{~Hz}), 111.3,53.6,36.0(\mathrm{~d}, J=67.7$ $\mathrm{Hz}), 27.7(\mathrm{~d}, J=1.5 \mathrm{~Hz}) .{ }^{31} \mathrm{P}$ NMR $\left(162 \mathrm{MHz}, \mathrm{CDCl}_{3}\right) \delta 30.6 . \mathrm{HRMS}: \mathrm{m} / \mathrm{z}(\mathrm{ESI})$ 
calculated $[\mathrm{M}+\mathrm{H}]^{+}$363.1257, measured: 363.1257 .

${ }^{[\alpha]} \mathrm{D}^{29.3} 95.4133\left(\mathrm{c} 1.0, \mathrm{CHCl}_{3}\right)$. HPLC (IF-3, $0.46 * 15 \mathrm{~cm}, 3 \mu \mathrm{m}$, hexane/ isopropanol $=6 / 4$, flow $0.7 \mathrm{~mL} / \mathrm{min}$, detection at $214 \mathrm{~nm}$ ) retention time $=17.178 \mathrm{~min}$ (major) and 19.170 min (minor).

$3 \mathbf{v}$

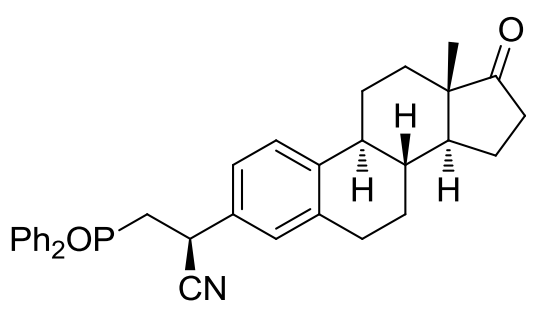

White solid. mp $147{ }^{\circ} \mathrm{C}-148{ }^{\circ} \mathrm{C}$.

${ }^{1} \mathrm{H}$ NMR (400 MHz, $\left.\mathrm{CDCl}_{3}\right) \delta 7.81-7.71(\mathrm{~m}, 2 \mathrm{H}), 7.60-7.46(\mathrm{~m}, 5 \mathrm{H}), 7.44-7.33$ (m, 1H), $7.36-7.29(\mathrm{~m}, 2 \mathrm{H}), 7.20-7.04(\mathrm{~m}, 2 \mathrm{H}), 6.97(\mathrm{~s}, 1 \mathrm{H}), 4.70-4.20(\mathrm{~m}, 1 \mathrm{H})$, $3.06-2.93(\mathrm{~m}, 1 \mathrm{H}), 2.87-2.69(\mathrm{~m}, 3 \mathrm{H}), 2.55-2.45(\mathrm{~m}, 1 \mathrm{H}), 2.37-2.28(\mathrm{~m}, 1 \mathrm{H})$, $2.18-1.93(\mathrm{~m}, 5 \mathrm{H}), 1.67-1.57(\mathrm{~m}, 1 \mathrm{H}), 1.55-1.49(\mathrm{~m}, 4 \mathrm{H}), 1.39-1.29(\mathrm{~m}, 1 \mathrm{H})$, $0.90(\mathrm{~s}, 3 \mathrm{H}) .{ }^{13} \mathrm{C} \mathrm{NMR}\left(100 \mathrm{MHz}, \mathrm{CDCl}_{3}\right) \delta 220.6,140.0,137.2,132.4(\mathrm{~d}, J=7.9 \mathrm{~Hz})$, $131.8(\mathrm{~d}, J=100.2 \mathrm{~Hz}), 131.8(\mathrm{~d}, J=100.4 \mathrm{~Hz}), 131.5(\mathrm{~d}, J=1.7 \mathrm{~Hz}), 130.8(\mathrm{~d}, J=$ $10.4 \mathrm{~Hz}), 130.4(\mathrm{~d}, J=9.7 \mathrm{~Hz}), 128.8(\mathrm{~d}, J=11.3 \mathrm{~Hz}), 128.4(\mathrm{~d}, J=12.1 \mathrm{~Hz}), 128.3$, 126.2, 124.7, 120.0 (d, $J=11.2 \mathrm{~Hz}), 50.3,47.8,44.2,37.8,36.1(\mathrm{~d}, J=69.3 \mathrm{~Hz}), 31.4$, $30.1(\mathrm{~d}, J=2.1 \mathrm{~Hz}), 29.2,29.1,28.2,25.5,21.5,13.7 .{ }^{31} \mathrm{P} \mathrm{NMR}\left(162 \mathrm{MHz}, \mathrm{CDCl}_{3}\right) \delta$ 27.4 HRMS: m/z (ESI) calculated $[\mathrm{M}+\mathrm{H}]^{+}$508.2400, measured: 508.2400.

${ }^{[\alpha]} \mathrm{D}^{29.1} 118.2533\left(\mathrm{c} 1.0, \mathrm{CHCl}_{3}\right)$. HPLC (IG, $0.46 * 25 \mathrm{~cm}, 3 \mu \mathrm{m}$, hexane/ isopropanol = $6 / 4$, flow $0.7 \mathrm{~mL} / \mathrm{min}$, detection at $214 \mathrm{~nm}$ ) retention time $=62.238 \mathrm{~min}$ (major) and 93.067 min (minor).

3a'

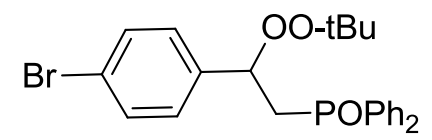

White solid. mp $151{ }^{\circ} \mathrm{C}-152{ }^{\circ} \mathrm{C}$.

${ }^{1} \mathrm{H}$ NMR $\left(400 \mathrm{MHz}, \mathrm{CDCl}_{3}\right) \delta 7.92-7.73(\mathrm{~m}, 2 \mathrm{H}), 7.65-7.45(\mathrm{~m}, 5 \mathrm{H}), 7.44-7.38$ (m, 1H), $7.37-7.28(\mathrm{~m}, 4 \mathrm{H}), 7.17(\mathrm{~d}, J=8.4 \mathrm{~Hz}, 2 \mathrm{H}), 5.40(\mathrm{q}, J=6.8 \mathrm{~Hz}, 1 \mathrm{H}), 3.14$ 
$-3.04(\mathrm{~m}, 1 \mathrm{H}), 2.66-2.53(\mathrm{~m}, 1 \mathrm{H}), 1.02(\mathrm{~s}, 9 \mathrm{H}) .{ }^{13} \mathrm{C} \mathrm{NMR}\left(100 \mathrm{MHz}, \mathrm{CDCl}_{3}\right) \delta$ $139.1(\mathrm{~d}, J=6.4 \mathrm{~Hz}), 131.7(\mathrm{~d}, J=2.8 \mathrm{~Hz}), 131.4(\mathrm{~d}, J=2.9 \mathrm{~Hz}), 131.3,130.8(\mathrm{~d}, J=$ $9.3 \mathrm{~Hz}), 130.4(\mathrm{~d}, J=9.7 \mathrm{~Hz}), 128.8,128.6(\mathrm{~d}, J=12.1 \mathrm{~Hz}), 128.4(\mathrm{~d}, J=11.7 \mathrm{~Hz})$, 122.0, 80.8, 79.6, $35.8(\mathrm{~d}, J=69.0 \mathrm{~Hz}), 26.2 .{ }^{31} \mathrm{P} \mathrm{NMR}\left(162 \mathrm{MHz}, \mathrm{CDCl}_{3}\right) \delta 27.7$ HRMS: m/z (ESI) calculated $[\mathrm{M}+\mathrm{H}]^{+}$473.0876, measured: 473.0877..

$4 a$

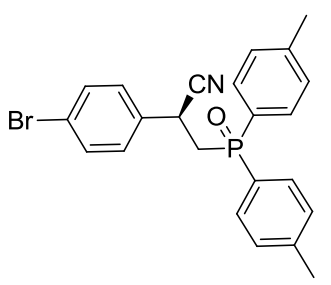

White solid. mp $148{ }^{\circ} \mathrm{C}-149^{\circ} \mathrm{C}$.

${ }^{1} \mathrm{H}$ NMR (400 MHz, $\left.\mathrm{CDCl}_{3}\right) \delta 7.64-7.57(\mathrm{~m}, 2 \mathrm{H}), 7.40-7.33(\mathrm{~m}, 2 \mathrm{H}), 7.52-7.44$ (m, 4H), $7.31-7.26(\mathrm{~m}, 2 \mathrm{H}), 7.17-7.10(\mathrm{~m}, 2 \mathrm{H}), 4.50-4.35(\mathrm{~m}, 1 \mathrm{H}), 3.02-2.85$ $(\mathrm{m}, 1 \mathrm{H}), 2.80-2.65(\mathrm{~m}, 1 \mathrm{H}), 2.38(\mathrm{~s}, 3 \mathrm{H}), 2.36(\mathrm{~s}, 3 \mathrm{H}) .{ }^{13} \mathrm{C} \mathrm{NMR}\left(100 \mathrm{MHz}, \mathrm{CDCl}_{3}\right)$ $\delta 143.0(\mathrm{~d}, J=3.8 \mathrm{~Hz}), 142.6(\mathrm{~d}, J=2.6 \mathrm{~Hz}), 134.0(\mathrm{~d}, J=5.9 \mathrm{~Hz}), 132.0,130.7(\mathrm{~d}, J$ $=9.9 \mathrm{~Hz}), 130.5(\mathrm{~d}, J=10.2 \mathrm{~Hz}), 129.6(\mathrm{~d}, J=12.0 \mathrm{~Hz}), 129.3,129.2,128.2(\mathrm{~d}, J=$ $102.8 \mathrm{~Hz}), 128.0$ (d, $J=103.5 \mathrm{~Hz}), 122.6,119.7$ (d, $J=10.4 \mathrm{~Hz}), 36.0$ (d, $J=68.7$ $\mathrm{Hz}), 30.2(\mathrm{~d}, J=2.3 \mathrm{~Hz}), 21.6,21.5 .{ }^{31} \mathrm{P} \mathrm{NMR}\left(162 \mathrm{MHz}, \mathrm{CDCl}_{3}\right) \delta 27.9$. HRMS: m/z (ESI) calculated $[\mathrm{M}+\mathrm{H}]^{+}$438.0617, measured: 438.0617.

${ }^{[\alpha]} \mathrm{D}^{23.8} 70.1946\left(\mathrm{c} 0.5, \mathrm{CHCl}_{3}\right)$. HPLC (IC-3, 0.46*15 cm, $3 \mu \mathrm{m}$, hexane/ isopropanol $=6 / 4$, flow $0.7 \mathrm{~mL} / \mathrm{min}$, detection at $214 \mathrm{~nm}$ ) retention time $=14.457 \mathrm{~min}$ (major) and $19.087 \min$ (minor).

$4 \mathbf{b}$

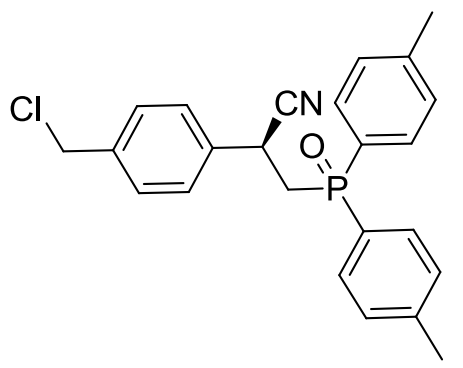

White solid. mp $146{ }^{\circ} \mathrm{C}-147^{\circ} \mathrm{C}$. 
${ }^{1} \mathrm{H}$ NMR (400 MHz, $\left.\mathrm{CDCl}_{3}\right) \delta 7.67-7.59(\mathrm{~m}, 2 \mathrm{H}), 7.44-7.36(\mathrm{~m}, 2 \mathrm{H}), 7.34-7.26$ (m, 4H), $7.22(\mathrm{~d}, J=8.4 \mathrm{~Hz}, 2 \mathrm{H}), 7.13(\mathrm{dd}, J=8.0,2.8 \mathrm{~Hz}, 2 \mathrm{H}), 4.55-4.35(\mathrm{~m}, 3 \mathrm{H})$, $3.02-2.90(\mathrm{~m}, 1 \mathrm{H}), 2.78-2.67(\mathrm{~m}, 1 \mathrm{H}), 2.39(\mathrm{~s}, 3 \mathrm{H}), 2.33(\mathrm{~s}, \mathrm{H}) .{ }^{13} \mathrm{C}$ NMR $(100$ $\left.\mathrm{MHz}, \mathrm{CDCl}_{3}\right) \delta 143.0(\mathrm{~d}, J=3.5 \mathrm{~Hz}), 142.4(\mathrm{~d}, J=2.6 \mathrm{~Hz}), 137.7,135.5(\mathrm{~d}, J=5.8$ $\mathrm{Hz}), 130.8$ (d, $J=10.1 \mathrm{~Hz}), 130.4(\mathrm{~d}, J=9.8 \mathrm{~Hz}), 129.6$ (d, $J=12.0 \mathrm{~Hz}), 129.3$ (d, $J$ $=12.3 \mathrm{~Hz}), 129.2,128.3(\mathrm{~d}, J=103.6 \mathrm{~Hz}), 128.3(\mathrm{~d}, J=103.3 \mathrm{~Hz}), 128.0,119.8(\mathrm{~d}, J$ $=10.0 \mathrm{~Hz}), 45.3,36.2(\mathrm{~d}, J=67.5 \mathrm{~Hz}), 30.3(\mathrm{~d}, J=2.6 \mathrm{~Hz}), 21.6,21.5 .{ }^{31} \mathrm{P}$ NMR $(162$ $\left.\mathrm{MHz}, \mathrm{CDCl}_{3}\right) \delta 27.9 \mathrm{HRMS}: \mathrm{m} / \mathrm{z}$ (ESI) calculated $[\mathrm{M}+\mathrm{H}]^{+}$408.1279, measured: 408.1280.

${ }^{[\alpha]} \mathrm{D}^{23.3} 25.8759\left(\mathrm{c} 0.5, \mathrm{CHCl}_{3}\right)$. HPLC (IC-3, 0.46*15 cm, $3 \mu \mathrm{m}$, hexane/ isopropanol $=6 / 4$, flow $0.7 \mathrm{~mL} / \mathrm{min}$, detection at $214 \mathrm{~nm}$ ) retention time $=18.398 \mathrm{~min}$ (major) and $23.483 \mathrm{~min}$ (minor).

$4 c$

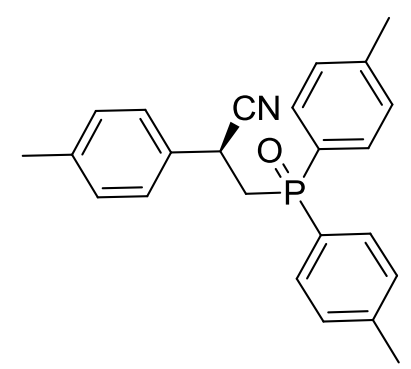

White solid. mp $143{ }^{\circ} \mathrm{C}-144{ }^{\circ} \mathrm{C}$.

${ }^{1} \mathrm{H}$ NMR $\left(400 \mathrm{MHz}, \mathrm{CDCl}_{3}\right) \delta 7.69-7.62(\mathrm{~m}, 2 \mathrm{H}), 7.44(\mathrm{dd}, J=11.2,8.0 \mathrm{~Hz}, 2 \mathrm{H})$, $7.33-7.28(\mathrm{~m}, 2 \mathrm{H}), 7.20-7.12(\mathrm{~m}, 4 \mathrm{H}), 7.03(\mathrm{~d}, J=7.8 \mathrm{~Hz}, 2 \mathrm{H}), 4.45-4.30(\mathrm{~m}$, $1 \mathrm{H}), 3.01-2.97(\mathrm{~m}, 1 \mathrm{H}), 2.75-2.65(\mathrm{~m}, 1 \mathrm{H}), 2.40(\mathrm{~s}, 3 \mathrm{H}), 2.34(\mathrm{~s}, 3 \mathrm{H}), 2.27(\mathrm{~s}, 3 \mathrm{H})$. ${ }^{13} \mathrm{C}$ NMR $\left(100 \mathrm{MHz}, \mathrm{CDCl}_{3}\right) \delta 142.9(\mathrm{~d}, J=2.8 \mathrm{~Hz}), 142.3(\mathrm{~d}, J=2.5 \mathrm{~Hz}), 138.1$, 132.5 (d, $J=7.1 \mathrm{~Hz}), 130.9$ (d, $J=10.2 \mathrm{~Hz}), 130.5$ (d, $J=9.9 \mathrm{~Hz}), 129.6,129.5$ (d, $J$ $=12.4 \mathrm{~Hz}), 129.2(\mathrm{~d}, J=12.4 \mathrm{~Hz}), 128.2(\mathrm{~d}, J=101.8 \mathrm{~Hz}), 128.1(\mathrm{~d}, J=101.8 \mathrm{~Hz})$, 127.3, $120.0(\mathrm{~d}, J=8.5 \mathrm{~Hz}), 36.4(\mathrm{~d}, J=67.8 \mathrm{~Hz}), 30.1(\mathrm{~d}, J=2.4 \mathrm{~Hz}), 21.6,21.5$, 20.9. ${ }^{31} \mathrm{P} \mathrm{NMR}\left(162 \mathrm{MHz}, \mathrm{CDCl}_{3}\right) \delta 27.9 \mathrm{HRMS}: \mathrm{m} / \mathrm{z}$ (EI) calculated $[\mathrm{M}]^{+}$373.1590, measured: 373.1590 .

${ }^{[\alpha]} \mathrm{D}^{28.8} 190.4347\left(\mathrm{c} 0.5, \mathrm{CHCl}_{3}\right)$. HPLC (IC-3, 0.46*15 cm, $3 \mu \mathrm{m}$, hexane/ isopropanol 
$=6 / 4$, flow $0.7 \mathrm{~mL} / \mathrm{min}$, detection at $214 \mathrm{~nm}$ ) retention time $=15.653 \mathrm{~min}$ (major) and $17.233 \mathrm{~min}$ (minor).

4d<smiles>Cc1cccc(P(=O)(CC(C)c2ccc3ccccc3c2)c2cccc(C)c2)c1</smiles>

White solid. mp $137{ }^{\circ} \mathrm{C}-138^{\circ} \mathrm{C}$.

${ }^{1} \mathrm{H}$ NMR (400 MHz, $\left.\mathrm{CDCl}_{3}\right) \delta 7.75-7.66(\mathrm{~m}, 4 \mathrm{H}), 7.62$ (d, $\left.J=12.4 \mathrm{~Hz}, 1 \mathrm{H}\right), 7.53-$ $7.44(\mathrm{~m}, 3 \mathrm{H}), 7.40-7.33(\mathrm{~m}, 3 \mathrm{H}), 7.30-7.19(\mathrm{~m}, 2 \mathrm{H}), 7.08-7.00(\mathrm{~m}, 1 \mathrm{H}), 6.96(\mathrm{~d}$, $J=7.6 \mathrm{~Hz} 1 \mathrm{H}), 4.70-4.56(\mathrm{~m}, 1 \mathrm{H}), 3.13-3.03(\mathrm{~m}, 1 \mathrm{H}), 2.91-2.81(\mathrm{~m}, 1 \mathrm{H}), 2.39$ (s, 3H), $2.04(\mathrm{~s}, 3 \mathrm{H}) .{ }^{13} \mathrm{C} \mathrm{NMR}\left(100 \mathrm{MHz}, \mathrm{CDCl}_{3}\right) \delta 138.8(\mathrm{~d}, J=10.9 \mathrm{~Hz}), 138.3(\mathrm{~d}, J=$ $11.1 \mathrm{~Hz}), 133.1$ (d, $J=3.3 \mathrm{~Hz}), 133.0,132.8,132.2,132.0$ (d, $J=5.0 \mathrm{~Hz}), 131.7$ (d, $J$ $=100.4 \mathrm{~Hz}), 131.1(\mathrm{~d}, J=100.3 \mathrm{~Hz}), 132.3(\mathrm{~d}, J=2.3 \mathrm{~Hz}), 131.2(\mathrm{~d}, J=9.2 \mathrm{~Hz})$, $130.9(\mathrm{~d}, J=9.2 \mathrm{~Hz}), 129.0,128.7(\mathrm{~d}, J=13.2 \mathrm{~Hz}), 128.1(\mathrm{~d}, J=13.5 \mathrm{~Hz}), 128.0$, $127.7(\mathrm{~d}, J=9.9 \mathrm{~Hz}), 127.6,127.4,127.3$ (d, $J=10.0 \mathrm{~Hz}), 126.7,126.6,124.5,120.1$ $(\mathrm{d}, J=11.8 \mathrm{~Hz}), 36.0(\mathrm{~d}, J=68.2 \mathrm{~Hz}), 30.8(\mathrm{~d}, J=2.3 \mathrm{~Hz}), 21.4,21.0 .{ }^{31} \mathrm{P}$ NMR $(162$ $\left.\mathrm{MHz}, \mathrm{CDCl}_{3}\right) \delta 27.8$ HRMS: m/z (EI) calculated $[\mathrm{M}+\mathrm{H}]^{+}$410.1668, measured: 410.1668.

${ }^{[\alpha]} \mathrm{D}^{29.2} 194.4211\left(\mathrm{c} 0.5, \mathrm{CHCl}_{3}\right)$. HPLC (IF-3, 0.46*15 cm, $3 \mu \mathrm{m}$, hexane/ isopropanol $=6 / 4$, flow $0.7 \mathrm{~mL} / \mathrm{min}$, detection at $214 \mathrm{~nm}$ ) retention time $=21.85 \mathrm{~min}$ (major) and $26.57 \mathrm{~min}$ (minor).

$4 e$

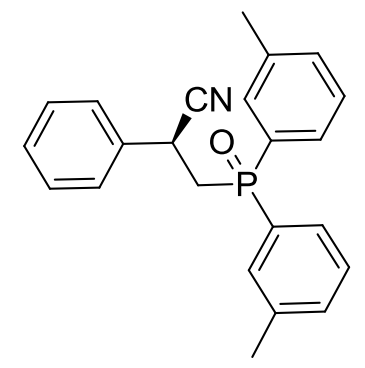

White solid. mp $138{ }^{\circ} \mathrm{C}-139{ }^{\circ} \mathrm{C}$. 
${ }^{1} \mathrm{H}$ NMR (400 MHz, $\left.\mathrm{CDCl}_{3}\right) \delta 7.63(\mathrm{~d}, J=12.4 \mathrm{~Hz}, 1 \mathrm{H}), 7.56-7.47(\mathrm{~m}, 1 \mathrm{H}), 7.40-$ $7.28(\mathrm{~m}, 6 \mathrm{H}), 7.25-7.15(\mathrm{~m}, 5 \mathrm{H}), 4.49-4.35(\mathrm{~m}, 1 \mathrm{H}), 3.05-2.95(\mathrm{~m}, 1 \mathrm{H}), 2.80-$ $2.70(\mathrm{~m}, 1 \mathrm{H}), 2.40(\mathrm{~s}, 3 \mathrm{H}), 2.28(\mathrm{~s}, 3 \mathrm{H}) .{ }^{13} \mathrm{C} \mathrm{NMR}\left(100 \mathrm{MHz}, \mathrm{CDCl}_{3}\right) \delta 138.8(\mathrm{~d}, J=$ 12.6 Hz), 138.5 (d, $J=12.7 \mathrm{~Hz}), 135.4(\mathrm{~d}, J=7.3 \mathrm{~Hz}), 133.1$ (d, $J=1.8 \mathrm{~Hz}), 132.7$ (d, $J=2.1 \mathrm{~Hz}), 131.6(\mathrm{~d}, J=102.0 \mathrm{~Hz}), 131.4(\mathrm{~d}, J=99.0 \mathrm{~Hz}), 131.3(\mathrm{~d}, J=9.8 \mathrm{~Hz})$, $131.0(\mathrm{~d}, J=9.0 \mathrm{~Hz}), 129.0,128.6(\mathrm{~d}, J=11.7 \mathrm{~Hz}), 128.4(\mathrm{~d}, J=13.1 \mathrm{~Hz}), 128.3$, $127.7(\mathrm{~d}, J=10.0 \mathrm{~Hz}), 127.5,127.4(\mathrm{~d}, J=10.2 \mathrm{~Hz}), 119.9$ (d, $J=9.1 \mathrm{~Hz}), 36.1$ (d, $J$ $=67.3 \mathrm{~Hz}), 30.5(\mathrm{~d}, J=3.1 \mathrm{~Hz}), 21.4,21.3 .{ }^{31} \mathrm{P} \mathrm{NMR}\left(162 \mathrm{MHz}, \mathrm{CDCl}_{3}\right) \delta 27.9$ HRMS: m/z (EI) calculated [M+H] 360.1512 , measured: 360.1513 .

${ }^{[\alpha]} \mathrm{D}^{29.2} 124.1515\left(\mathrm{c} 0.8, \mathrm{CHCl}_{3}\right)$. HPLC (IF-3, 0.46*15 cm, $3 \mu \mathrm{m}$, hexane/ isopropanol $=6 / 4$, flow $0.7 \mathrm{~mL} / \mathrm{min}$, detection at $214 \mathrm{~nm}$ ) retention time $=31.39 \mathrm{~min}$ (major) and $35.77 \mathrm{~min}$ (minor).

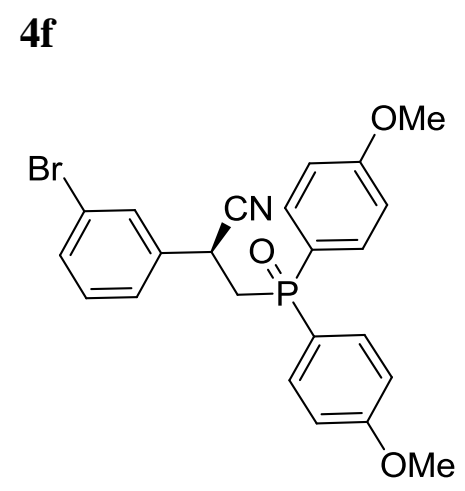

White solid. mp $131{ }^{\circ} \mathrm{C}-132{ }^{\circ} \mathrm{C}$.

${ }^{1} \mathrm{H}$ NMR (400 MHz, $\left.\mathrm{CDCl}_{3}\right) \delta 7.69-7.61(\mathrm{~m}, 2 \mathrm{H}), 7.49-7.41(\mathrm{~m}, 2 \mathrm{H}), 7.40-7.35$ (m, 1H), $7.32(\mathrm{~d}, J=7.2 \mathrm{~Hz}, 1 \mathrm{H}), 7.25(\mathrm{~d}, J=8.4 \mathrm{~Hz}, 1 \mathrm{H}), 7.10(\mathrm{~d}, J=8.0 \mathrm{~Hz}, 1 \mathrm{H})$, $7.02-6.95(\mathrm{~m}, 2 \mathrm{H}), 6.89-6.79(\mathrm{~m}, 2 \mathrm{H}), 4.44-4.34(\mathrm{~m}, 1 \mathrm{H}), 3.83(\mathrm{~s}, 3 \mathrm{H}), 3.81(\mathrm{~s}$, $3 \mathrm{H}), 3.00-2.89(\mathrm{~m}, 1 \mathrm{H}), 2.72-2.61(\mathrm{~m}, 1 \mathrm{H}) .{ }^{13} \mathrm{C} \mathrm{NMR}\left(100 \mathrm{MHz}, \mathrm{CDCl}_{3}\right) \delta 162.8$ $(\mathrm{d}, J=2.9 \mathrm{~Hz}), 162.3(\mathrm{~d}, J=3.4 \mathrm{~Hz}), 137.4(\mathrm{~d}, J=6.4 \mathrm{~Hz}), 132.7(\mathrm{~d}, J=11.0 \mathrm{~Hz})$, $132.3(\mathrm{~d}, J=11.6 \mathrm{~Hz}), 131.5,130.7,130.6,126.3,122.9,122.7(\mathrm{~d}, J=106.6 \mathrm{~Hz})$, $122.5(\mathrm{~d}, J=106.6 \mathrm{~Hz}), 119.5(\mathrm{~d}, J=10.1 \mathrm{~Hz}), 114.4(\mathrm{~d}, J=13.1 \mathrm{~Hz}), 114.2(\mathrm{~d}, J=$ 13.0 Hz), 55.3, 36.3 (d, $J=69.1 \mathrm{~Hz}), 30.3$ (d, $J=1.9 \mathrm{~Hz}) .{ }^{31} \mathrm{P}$ NMR (162 MHz, $\left.\mathrm{CDCl}_{3}\right) \delta$ 30.1. HRMS: m/z (EI) calculated $[\mathrm{M}+\mathrm{H}]^{+}$470.0515, measured: 470.0516 . ${ }^{[\alpha]} \mathrm{D}^{26.6} 170.3217$ (c $0.6, \mathrm{CHCl}_{3}$ ). HPLC (IC-3, 0.46*15 cm, $3 \mu \mathrm{m}$, hexane/ isopropanol 
$=6 / 4$, flow $0.7 \mathrm{~mL} / \mathrm{min}$, detection at $214 \mathrm{~nm}$ ) retention time $=33.545 \mathrm{~min}$ (major) and $49.598 \min$ (minor).

\section{$4 \mathrm{~g}$}

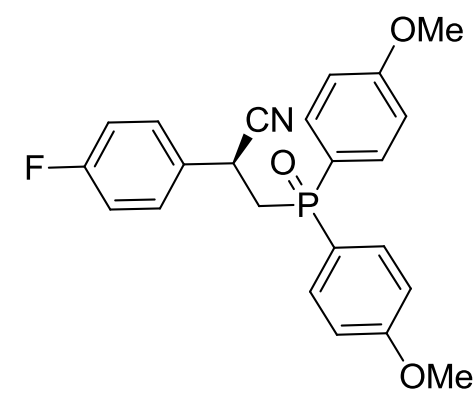

White solid. mp $134{ }^{\circ} \mathrm{C}-135{ }^{\circ} \mathrm{C}$.

${ }^{1} \mathrm{H}$ NMR (400 MHz, $\left.\mathrm{CDCl}_{3}\right) \delta 7.72-7.62(\mathrm{~m}, 2 \mathrm{H}), 7.50-7.40(\mathrm{~m}, 2 \mathrm{H}), 7.30-7.24$ $(\mathrm{m}, 2 \mathrm{H}), 7.04-6.96(\mathrm{~m}, 2 \mathrm{H}), 6.95-6.88(\mathrm{~m}, 2 \mathrm{H}), 6.88-6.80(\mathrm{~m}, 2 \mathrm{H}), 4.50-4.34$ $(\mathrm{m}, 1 \mathrm{H}), 3.84(\mathrm{~s}, 3 \mathrm{H}), 3.80(\mathrm{~s}, 3 \mathrm{H}), 3.03-2.89(\mathrm{~m}, 1 \mathrm{H}), 2.76-2.60(\mathrm{~m}, 1 \mathrm{H}) .{ }^{13} \mathrm{C}$ NMR (100 MHz, $\left.\mathrm{CDCl}_{3}\right) \delta 162.7(\mathrm{~d}, J=2.5 \mathrm{~Hz}), 162.3(\mathrm{~d}, J=546.7 \mathrm{~Hz}), 162.3(\mathrm{~d}, J$ $=1.9 \mathrm{~Hz}), 132.7(\mathrm{~d}, J=10.2 \mathrm{~Hz}), 132.3(\mathrm{~d}, J=11.0 \mathrm{~Hz}), 131.1(\mathrm{dd}, J=6.4,3.2 \mathrm{~Hz})$, $129.4(\mathrm{~d}, J=8.5 \mathrm{~Hz}), 122.8(\mathrm{~d}, J=107.4 \mathrm{~Hz}), 122.7(\mathrm{~d}, J=107.1 \mathrm{~Hz}), 119.9(\mathrm{~d}, J=$ $9.3 \mathrm{~Hz}), 116.0(\mathrm{~d}, J=12.2 \mathrm{~Hz}), 114.4(\mathrm{~d}, J=13.1 \mathrm{~Hz}), 114.1(\mathrm{~d}, J=12.9 \mathrm{~Hz}), 55.3$, $36.5(\mathrm{~d}, J=68.1 \mathrm{~Hz}), 30.0(\mathrm{~d}, J=1.7 \mathrm{~Hz}) .{ }^{31} \mathrm{P} \mathrm{NMR}\left(162 \mathrm{MHz}, \mathrm{CDCl}_{3}\right) \delta 31.0 \mathrm{HRMS}$ : $\mathrm{m} / \mathrm{z}$ (ESI) calculated $[\mathrm{M}+\mathrm{H}]^{+}$410.1316, measured: 410.1318 .

${ }^{[\alpha]} \mathrm{D}^{23.5} 69.0211\left(\mathrm{c} 0.5, \mathrm{CHCl}_{3}\right)$. HPLC (IC-3, 0.46*15 cm, $3 \mu \mathrm{m}$, hexane/ isopropanol $=6 / 4$, flow $0.7 \mathrm{~mL} / \mathrm{min}$, detection at $214 \mathrm{~nm}$ ) retention time $=35.545 \mathrm{~min}$ (major) and $49.598 \mathrm{~min}$ (minor).

\section{4h}

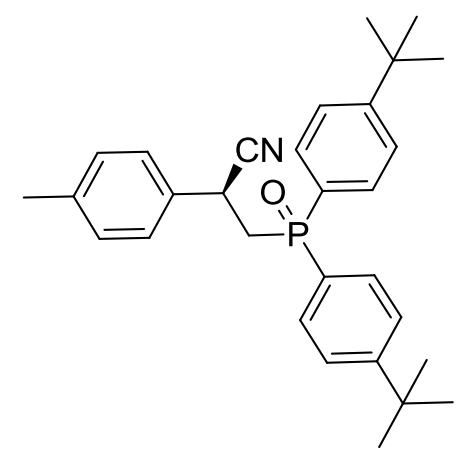

White solid. mp $141{ }^{\circ} \mathrm{C}-142{ }^{\circ} \mathrm{C}$. 
${ }^{1} \mathrm{H}$ NMR $\left(400 \mathrm{MHz}, \mathrm{CDCl}_{3}\right) \delta 7.71-7.64(\mathrm{~m}, 2 \mathrm{H}), 7.52-7.48(\mathrm{~m}, 2 \mathrm{H}), 7.47-7.41$ (m, 2H), $7.36-7.28(\mathrm{~m}, 2 \mathrm{H}), 7.15(\mathrm{~d}, J=8.4 \mathrm{~Hz}, 2 \mathrm{H}), 6.97(\mathrm{~d}, J=8.0 \mathrm{~Hz}, 2 \mathrm{H}), 4.48$ - $4.32(\mathrm{~m}, 1 \mathrm{H}), 3.03-2.93(\mathrm{~m}, 1 \mathrm{H}), 2.80-2.70(\mathrm{~m}, 1 \mathrm{H}), 2.24(\mathrm{~s}, 3 \mathrm{H}), 1.31(\mathrm{~s}, 9 \mathrm{H})$, $1.28(\mathrm{~s}, 9 \mathrm{H}) .{ }^{13} \mathrm{C}$ NMR $\left(100 \mathrm{MHz}, \mathrm{CDCl}_{3}\right) \delta 155.8(\mathrm{~d}, J=2.1 \mathrm{~Hz}), 155.2(\mathrm{~d}, J=3.0$ Hz), 140.0, $132.2(\mathrm{~d}, J=6.3 \mathrm{~Hz}), 130.6(\mathrm{~d}, J=10.1 \mathrm{~Hz}), 130.4(\mathrm{~d}, J=10.1 \mathrm{~Hz}), 129.5$, $128.7(\mathrm{~d}, J=101.8 \mathrm{~Hz}), 128.3(\mathrm{~d}, J=102.4 \mathrm{~Hz}), 127.5,125.8(\mathrm{~d}, J=12.5 \mathrm{~Hz}), 125.4$ $(\mathrm{d}, J=12.0 \mathrm{~Hz}), 120.4(\mathrm{~d}, J=10.7 \mathrm{~Hz}), 36.4(\mathrm{~d}, J=68.0 \mathrm{~Hz}), 34.9(\mathrm{~d}, J=12.5 \mathrm{~Hz})$, 31.0, $30.2(\mathrm{~d}, J=1.0 \mathrm{~Hz}), 21.0 .{ }^{31} \mathrm{P}$ NMR $\left(162 \mathrm{MHz}, \mathrm{CDCl}_{3}\right) \delta 27.6 . \mathrm{HRMS}: \mathrm{m} / \mathrm{z}(\mathrm{EI})$ calculated $[\mathrm{M}+\mathrm{H}]^{+}$458.2607, measured: 458.2607.

${ }^{[\alpha]} \mathrm{D}^{23.7} 8.0486\left(\mathrm{c} 0.5, \mathrm{CHCl}_{3}\right)$. HPLC (IC-3, 0.46*15 cm, $3 \mu \mathrm{m}$, hexane/ isopropanol = 6/4, flow $0.7 \mathrm{~mL} / \mathrm{min}$, detection at $214 \mathrm{~nm}$ ) retention time $=8.502 \mathrm{~min}$ (major) and $10.768 \min (\operatorname{minor})$.

4i

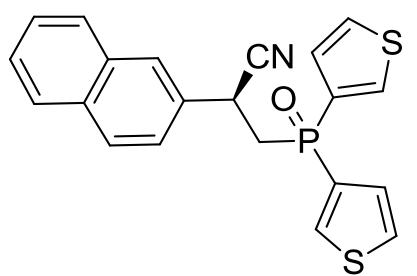

White solid. mp $148{ }^{\circ} \mathrm{C}-147^{\circ} \mathrm{C}$.

${ }^{1} \mathrm{H}$ NMR $\left(400 \mathrm{MHz}, \mathrm{CDCl}_{3}\right) \delta 7.98-7.78(\mathrm{~m}, 6 \mathrm{H}), 7.73-7.69(\mathrm{~m}, 1 \mathrm{H}), 7.56-7.52$ (m, 1H), $7.52-7.47(\mathrm{~m}, 2 \mathrm{H}), 7.45-7.38(\mathrm{~m}, 2 \mathrm{H}), 6.92-6.98(\mathrm{~m}, 1 \mathrm{H}), 4.70-4.58$ $(\mathrm{m}, 1 \mathrm{H}), 3.16-3.06(\mathrm{~m}, 1 \mathrm{H}), 2.93-2.82(\mathrm{~m}, 1 \mathrm{H}) .{ }^{13} \mathrm{C} \mathrm{NMR}\left(100 \mathrm{MHz}, \mathrm{CDCl}_{3}\right) \delta$ $136.5(\mathrm{~d}, J=10.2 \mathrm{~Hz}), 135.9$ (d, $J=9.4 \mathrm{~Hz}), 134.4(\mathrm{~d}, J=5.1 \mathrm{~Hz}), 133.8(\mathrm{~d}, J=5.6$ $\mathrm{Hz}), 133.1,132.9,132.4(\mathrm{~d}, J=116.5 \mathrm{~Hz}), 132.2(\mathrm{~d}, J=116.1 \mathrm{~Hz}), 132.2(\mathrm{~d}, J=8.7$ $\mathrm{Hz}), 129.4,128.7$ (d, $J=14.5 \mathrm{~Hz}), 128.3$ (d, $J=14.7 \mathrm{~Hz}), 127.9,127.6,127.0,126.7$, 124.4, 119.5 (d, $J=9.2 \mathrm{~Hz}), 40.2(\mathrm{~d}, J=76.2 \mathrm{~Hz}), 30.9$ (d, $J=2.8 \mathrm{~Hz}) .{ }^{31} \mathrm{P}$ NMR $(162$ $\left.\mathrm{MHz}, \mathrm{CDCl}_{3}\right) \delta 27.6$ HRMS: m/z (EI) calculated $[\mathrm{M}+\mathrm{H}]^{+}$394.0484, measured: 394.0485 .

${ }^{[\alpha]} \mathrm{D}^{26.7} 17.6955$ (c $0.8, \mathrm{CHCl}_{3}$ ). HPLC (IF-3, 0.46*15 cm, $3 \mu \mathrm{m}$, hexane/ isopropanol $=6 / 4$, flow $0.7 \mathrm{~mL} / \mathrm{min}$, detection at $214 \mathrm{~nm}$ ) retention time $=8.502 \mathrm{~min}$ (major) and 
$10.768 \min$ (minor).

\section{X-Ray Data (3a)}

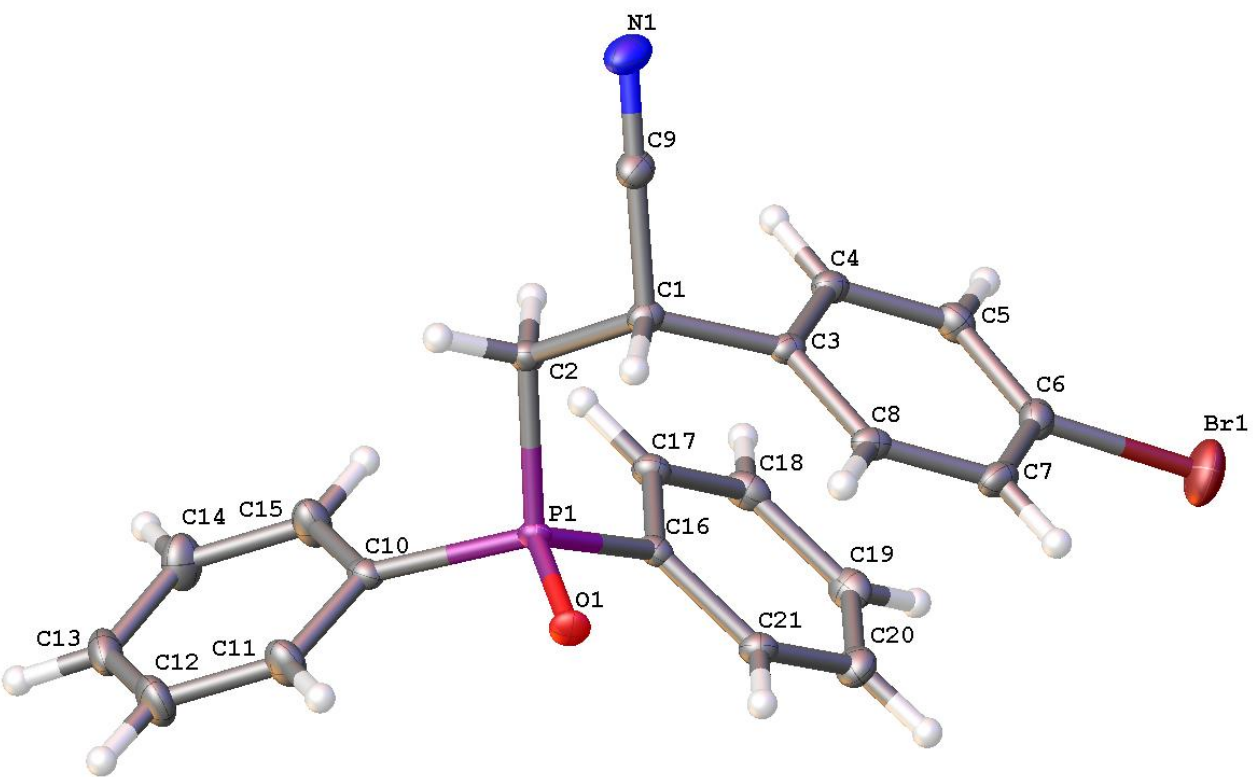

Table 1. Crystal data and structure refinement for $\mathrm{m} J 118160 \_0 \mathrm{~m}$.

Identification code

Empirical formula

Formula weight

Temperature

Wavelength

Crystal system

Space group

Unit cell dimensions

Volume

Z

Density (calculated)

Absorption coefficient

$\mathrm{F}(000)$

Crystal size
mJ18160_0m

C21 H17 Br N O P

410.24

$186.5 \mathrm{~K}$

$1.34139 \AA$

Monoclinic

C 121

$a=25.1172(5) \AA$

$\alpha=90^{\circ}$.

$\mathrm{b}=5.75290(10) \AA$ $\beta=109.4600(10)^{\circ}$.

$c=13.5472(3) \AA$ $\gamma=90^{\circ}$.
4

$1.476 \mathrm{Mg} / \mathrm{m}^{3}$

$2.653 \mathrm{~mm}^{-1}$

832

$0.15 \times 0.05 \times 0.03 \mathrm{~mm}^{3}$ 
Theta range for data collection

Index ranges

Reflections collected

Independent reflections

Completeness to theta $=53.594^{\circ}$

Absorption correction

Max. and min. transmission

Refinement method

Data / restraints / parameters

Goodness-of-fit on $\mathrm{F}^{2}$

Final $\mathrm{R}$ indices [I $>2 \operatorname{sigma}(\mathrm{I})]$

$\mathrm{R}$ indices (all data)

Absolute structure parameter

Extinction coefficient

Largest diff. peak and hole
3.010 to $54.905^{\circ}$.

$-30<=\mathrm{h}<=30,-7<=\mathrm{k}<=6,-16<=\mathrm{l}<=16$

11764

$3412[\mathrm{R}(\mathrm{int})=0.0292]$

$99.5 \%$

Semi-empirical from equiv.alents

0.7508 and 0.5113

Full-matrix least-squares on $\mathrm{F}^{2}$

3412 / 1 / 226

1.062

$\mathrm{R} 1=0.0239, \mathrm{wR} 2=0.0564$

$\mathrm{R} 1=0.0255, \mathrm{wR} 2=0.0608$

$0.016(9)$

$\mathrm{n} / \mathrm{a}$

0.249 and -0.522 e. $\AA^{-3}$

Table 2. Atomic coordinates $\left(\mathrm{x} 10^{4}\right)$ and equiv.alent isotropic displacement parameters $\left(\AA^{2} \times 10^{3}\right)$ for $\mathrm{m} J 18160 \_0 \mathrm{~m} . \quad \mathrm{U}(\mathrm{eq})$ is defined as one third of the trace of the orthogonalized $\mathrm{U}^{\mathrm{i} J}$ tensor.

$\mathrm{y}$

Z

$\mathrm{U}(\mathrm{eq})$

\begin{tabular}{|c|c|c|c|c|}
\hline $\operatorname{Br}(1)$ & $7036(1)$ & $4897(1)$ & $113(1)$ & $55(1)$ \\
\hline $\mathrm{P}(1)$ & $5891(1)$ & $6607(1)$ & $3814(1)$ & $18(1)$ \\
\hline $\mathrm{O}(1)$ & $5712(1)$ & $8930(3)$ & $3334(2)$ & $24(1)$ \\
\hline $\mathrm{N}(1)$ & $4430(1)$ & $1112(6)$ & $1303(2)$ & $41(1)$ \\
\hline$C(1)$ & $5127(1)$ & $4585(5)$ & $2027(2)$ & $22(1)$ \\
\hline $\mathrm{C}(2)$ & $5378(1)$ & $4347(5)$ & $3239(2)$ & $20(1$ \\
\hline $\mathrm{C}(3)$ & $5574(1)$ & $4596(5)$ & $1496(2)$ & 0( \\
\hline $\mathrm{C}(4)$ & $5932(1)$ & $2705(5)$ & $1600(2)$ & $24(1)$ \\
\hline
\end{tabular}




\begin{tabular}{|c|c|c|c|c|}
\hline$C(5)$ & $6366(1)$ & $2777(6)$ & $1182(2)$ & $28(1)$ \\
\hline$C(6)$ & $6429(1)$ & $4750(7)$ & $647(2)$ & $29(1)$ \\
\hline$C(7)$ & $6071(1)$ & $6626(6)$ & $508(2)$ & $29(1)$ \\
\hline $\mathrm{C}(8)$ & $5641(1)$ & $6548(6)$ & $945(2)$ & $23(1)$ \\
\hline $\mathrm{C}(9)$ & $4728(1)$ & $2639(6)$ & $1615(2)$ & $28(1)$ \\
\hline $\mathrm{C}(10)$ & $5966(1)$ & $6614(6)$ & $5192(2)$ & $22(1)$ \\
\hline $\mathrm{C}(11)$ & $5757(2)$ & $8559(6)$ & $5551(3)$ & $35(1)$ \\
\hline $\mathrm{C}(12)$ & $5805(2)$ & $8751(7)$ & $6597(3)$ & $42(1)$ \\
\hline $\mathrm{C}(13)$ & $6064(1)$ & $7034(7)$ & $7292(2)$ & $36(1)$ \\
\hline $\mathrm{C}(14)$ & $6264(1)$ & $5079(8)$ & $6949(2)$ & $40(1)$ \\
\hline$C(15)$ & $6216(1)$ & $4870(7)$ & $5899(2)$ & $34(1)$ \\
\hline$C(16)$ & $6562(1)$ & $5716(5)$ & $3710(2)$ & $20(1)$ \\
\hline $\mathrm{C}(17)$ & $6813(1)$ & $3563(5)$ & $4044(2)$ & $23(1)$ \\
\hline $\mathrm{C}(18)$ & $7335(1)$ & $3036(6)$ & $3951(2)$ & $28(1)$ \\
\hline C(19) & $7600(1)$ & $4636(7)$ & $3499(2)$ & $30(1)$ \\
\hline $\mathrm{C}(20)$ & $7350(1)$ & $6741(7)$ & $3152(2)$ & $31(1)$ \\
\hline $\mathrm{C}(21)$ & $6832(1)$ & $7303(5)$ & $3263(2)$ & $23(1)$ \\
\hline
\end{tabular}

Table 3. Bond lengths $[\AA]$ and angles $\left[{ }^{\circ}\right]$ for $\quad m J 118160 \_0 \mathrm{~m}$.

\begin{tabular}{ll}
\hline $\mathrm{Br}(1)-\mathrm{C}(6)$ & $1.893(3)$ \\
$\mathrm{P}(1)-\mathrm{O}(1)$ & $1.489(2)$ \\
$\mathrm{P}(1)-\mathrm{C}(2)$ & $1.814(3)$ \\
$\mathrm{P}(1)-\mathrm{C}(10)$ & $1.814(3)$ \\
$\mathrm{P}(1)-\mathrm{C}(16)$ & $1.811(3)$ \\
$\mathrm{N}(1)-\mathrm{C}(9)$ & $1.140(4)$ \\
$\mathrm{C}(1)-\mathrm{H}(1)$ & 1.0000 \\
$\mathrm{C}(1)-\mathrm{C}(2)$ & $1.556(3)$ \\
$\mathrm{C}(1)-\mathrm{C}(3)$ & $1.522(3)$
\end{tabular}




\begin{tabular}{|c|c|}
\hline $\mathrm{C}(1)-\mathrm{C}(9)$ & $1.481(4)$ \\
\hline $\mathrm{C}(2)-\mathrm{H}(2 \mathrm{~A})$ & 0.9900 \\
\hline $\mathrm{C}(2)-\mathrm{H}(2 \mathrm{~B})$ & 0.9900 \\
\hline$C(3)-C(4)$ & $1.387(4)$ \\
\hline $\mathrm{C}(3)-\mathrm{C}(8)$ & $1.390(4)$ \\
\hline $\mathrm{C}(4)-\mathrm{H}(4)$ & 0.9500 \\
\hline$C(4)-C(5)$ & $1.389(4)$ \\
\hline $\mathrm{C}(5)-\mathrm{H}(5)$ & 0.9500 \\
\hline$C(5)-C(6)$ & $1.384(5)$ \\
\hline$C(6)-C(7)$ & $1.377(5)$ \\
\hline $\mathrm{C}(7)-\mathrm{H}(7)$ & 0.9500 \\
\hline$C(7)-C(8)$ & $1.394(4)$ \\
\hline $\mathrm{C}(8)-\mathrm{H}(8)$ & 0.9500 \\
\hline $\mathrm{C}(10)-\mathrm{C}(11)$ & $1.390(4)$ \\
\hline$C(10)-C(15)$ & $1.385(4)$ \\
\hline $\mathrm{C}(11)-\mathrm{H}(11)$ & 0.9500 \\
\hline $\mathrm{C}(11)-\mathrm{C}(12)$ & $1.386(5)$ \\
\hline $\mathrm{C}(12)-\mathrm{H}(12)$ & 0.9500 \\
\hline $\mathrm{C}(12)-\mathrm{C}(13)$ & $1.370(5)$ \\
\hline $\mathrm{C}(13)-\mathrm{H}(13)$ & 0.9500 \\
\hline $\mathrm{C}(13)-\mathrm{C}(14)$ & $1.374(6)$ \\
\hline $\mathrm{C}(14)-\mathrm{H}(14)$ & 0.9500 \\
\hline$C(14)-C(15)$ & $1.392(4)$ \\
\hline $\mathrm{C}(15)-\mathrm{H}(15)$ & 0.9500 \\
\hline$C(16)-C(17)$ & $1.395(4)$ \\
\hline$C(16)-C(21)$ & $1.390(4)$ \\
\hline $\mathrm{C}(17)-\mathrm{H}(17)$ & 0.9500 \\
\hline $\mathrm{C}(17)-\mathrm{C}(18)$ & $1.391(4)$ \\
\hline $\mathrm{C}(18)-\mathrm{H}(18)$ & 0.9500 \\
\hline
\end{tabular}




\begin{tabular}{|c|c|}
\hline $\mathrm{C}(18)-\mathrm{C}(19)$ & $1.392(5)$ \\
\hline C(19)-H(19) & 0.9500 \\
\hline $\mathrm{C}(19)-\mathrm{C}(20)$ & $1.373(5)$ \\
\hline $\mathrm{C}(20)-\mathrm{H}(20)$ & 0.9500 \\
\hline $\mathrm{C}(20)-\mathrm{C}(21)$ & $1.397(4)$ \\
\hline $\mathrm{C}(21)-\mathrm{H}(21)$ & 0.9500 \\
\hline $\mathrm{O}(1)-\mathrm{P}(1)-\mathrm{C}(2)$ & $112.83(12)$ \\
\hline $\mathrm{O}(1)-\mathrm{P}(1)-\mathrm{C}(10)$ & $111.05(13)$ \\
\hline $\mathrm{O}(1)-\mathrm{P}(1)-\mathrm{C}(16)$ & $111.98(13)$ \\
\hline $\mathrm{C}(10)-\mathrm{P}(1)-\mathrm{C}(2)$ & $104.96(13)$ \\
\hline $\mathrm{C}(16)-\mathrm{P}(1)-\mathrm{C}(2)$ & $108.13(13)$ \\
\hline $\mathrm{C}(16)-\mathrm{P}(1)-\mathrm{C}(10)$ & $107.50(12)$ \\
\hline $\mathrm{C}(2)-\mathrm{C}(1)-\mathrm{H}(1)$ & 108.4 \\
\hline $\mathrm{C}(3)-\mathrm{C}(1)-\mathrm{H}(1)$ & 108.4 \\
\hline $\mathrm{C}(3)-\mathrm{C}(1)-\mathrm{C}(2)$ & $113.3(2)$ \\
\hline $\mathrm{C}(9)-\mathrm{C}(1)-\mathrm{H}(1)$ & 108.4 \\
\hline $\mathrm{C}(9)-\mathrm{C}(1)-\mathrm{C}(2)$ & $108.5(2)$ \\
\hline $\mathrm{C}(9)-\mathrm{C}(1)-\mathrm{C}(3)$ & $109.7(2)$ \\
\hline $\mathrm{P}(1)-\mathrm{C}(2)-\mathrm{H}(2 \mathrm{~A})$ & 109.3 \\
\hline $\mathrm{P}(1)-\mathrm{C}(2)-\mathrm{H}(2 \mathrm{~B})$ & 109.3 \\
\hline $\mathrm{C}(1)-\mathrm{C}(2)-\mathrm{P}(1)$ & $111.72(19)$ \\
\hline $\mathrm{C}(1)-\mathrm{C}(2)-\mathrm{H}(2 \mathrm{~A})$ & 109.3 \\
\hline $\mathrm{C}(1)-\mathrm{C}(2)-\mathrm{H}(2 \mathrm{~B})$ & 109.3 \\
\hline $\mathrm{H}(2 \mathrm{~A})-\mathrm{C}(2)-\mathrm{H}(2 \mathrm{~B})$ & 107.9 \\
\hline $\mathrm{C}(4)-\mathrm{C}(3)-\mathrm{C}(1)$ & $120.2(3)$ \\
\hline $\mathrm{C}(4)-\mathrm{C}(3)-\mathrm{C}(8)$ & $119.8(2)$ \\
\hline $\mathrm{C}(8)-\mathrm{C}(3)-\mathrm{C}(1)$ & $119.9(3)$ \\
\hline $\mathrm{C}(3)-\mathrm{C}(4)-\mathrm{H}(4)$ & 119.8 \\
\hline
\end{tabular}




\begin{tabular}{|c|c|}
\hline$C(3)-C(4)-C(5)$ & $120.4(3)$ \\
\hline $\mathrm{C}(5)-\mathrm{C}(4)-\mathrm{H}(4)$ & 119.8 \\
\hline $\mathrm{C}(4)-\mathrm{C}(5)-\mathrm{H}(5)$ & 120.6 \\
\hline$C(6)-C(5)-C(4)$ & $118.7(3)$ \\
\hline $\mathrm{C}(6)-\mathrm{C}(5)-\mathrm{H}(5)$ & 120.6 \\
\hline $\mathrm{C}(5)-\mathrm{C}(6)-\mathrm{Br}(1)$ & $118.9(3)$ \\
\hline $\mathrm{C}(7)-\mathrm{C}(6)-\mathrm{Br}(1)$ & $119.0(3)$ \\
\hline$C(7)-C(6)-C(5)$ & $122.1(3)$ \\
\hline $\mathrm{C}(6)-\mathrm{C}(7)-\mathrm{H}(7)$ & 120.7 \\
\hline$C(6)-C(7)-C(8)$ & $118.7(3)$ \\
\hline $\mathrm{C}(8)-\mathrm{C}(7)-\mathrm{H}(7)$ & 120.7 \\
\hline $\mathrm{C}(3)-\mathrm{C}(8)-\mathrm{C}(7)$ & $120.3(3)$ \\
\hline $\mathrm{C}(3)-\mathrm{C}(8)-\mathrm{H}(8)$ & 119.9 \\
\hline $\mathrm{C}(7)-\mathrm{C}(8)-\mathrm{H}(8)$ & 119.9 \\
\hline $\mathrm{N}(1)-\mathrm{C}(9)-\mathrm{C}(1)$ & $178.7(3)$ \\
\hline $\mathrm{C}(11)-\mathrm{C}(10)-\mathrm{P}(1)$ & $116.2(2)$ \\
\hline $\mathrm{C}(15)-\mathrm{C}(10)-\mathrm{P}(1)$ & $125.3(2)$ \\
\hline$C(15)-C(10)-C(11)$ & $118.5(3)$ \\
\hline $\mathrm{C}(10)-\mathrm{C}(11)-\mathrm{H}(11)$ & 119.7 \\
\hline$C(12)-C(11)-C(10)$ & $120.5(3)$ \\
\hline $\mathrm{C}(12)-\mathrm{C}(11)-\mathrm{H}(11)$ & 119.7 \\
\hline $\mathrm{C}(11)-\mathrm{C}(12)-\mathrm{H}(12)$ & 119.9 \\
\hline $\mathrm{C}(13)-\mathrm{C}(12)-\mathrm{C}(11)$ & $120.3(3)$ \\
\hline $\mathrm{C}(13)-\mathrm{C}(12)-\mathrm{H}(12)$ & 119.9 \\
\hline $\mathrm{C}(12)-\mathrm{C}(13)-\mathrm{H}(13)$ & 120.0 \\
\hline $\mathrm{C}(12)-\mathrm{C}(13)-\mathrm{C}(14)$ & $120.1(3)$ \\
\hline $\mathrm{C}(14)-\mathrm{C}(13)-\mathrm{H}(13)$ & 120.0 \\
\hline $\mathrm{C}(13)-\mathrm{C}(14)-\mathrm{H}(14)$ & 120.0 \\
\hline$C(13)-C(14)-C(15)$ & $119.9(4)$ \\
\hline
\end{tabular}




\begin{tabular}{|c|c|}
\hline $\mathrm{C}(15)-\mathrm{C}(14)-\mathrm{H}(14)$ & 120.0 \\
\hline$C(10)-C(15)-C(14)$ & $120.6(3)$ \\
\hline $\mathrm{C}(10)-\mathrm{C}(15)-\mathrm{H}(15)$ & 119.7 \\
\hline $\mathrm{C}(14)-\mathrm{C}(15)-\mathrm{H}(15)$ & 119.7 \\
\hline $\mathrm{C}(17)-\mathrm{C}(16)-\mathrm{P}(1)$ & $123.9(2)$ \\
\hline $\mathrm{C}(21)-\mathrm{C}(16)-\mathrm{P}(1)$ & $116.6(2)$ \\
\hline$C(21)-C(16)-C(17)$ & $119.5(3)$ \\
\hline $\mathrm{C}(16)-\mathrm{C}(17)-\mathrm{H}(17)$ & 120.0 \\
\hline $\mathrm{C}(18)-\mathrm{C}(17)-\mathrm{C}(16)$ & $120.1(3)$ \\
\hline $\mathrm{C}(18)-\mathrm{C}(17)-\mathrm{H}(17)$ & 120.0 \\
\hline $\mathrm{C}(17)-\mathrm{C}(18)-\mathrm{H}(18)$ & 120.0 \\
\hline $\mathrm{C}(17)-\mathrm{C}(18)-\mathrm{C}(19)$ & $119.9(3)$ \\
\hline $\mathrm{C}(19)-\mathrm{C}(18)-\mathrm{H}(18)$ & 120.0 \\
\hline $\mathrm{C}(18)-\mathrm{C}(19)-\mathrm{H}(19)$ & 119.9 \\
\hline $\mathrm{C}(20)-\mathrm{C}(19)-\mathrm{C}(18)$ & $120.1(3)$ \\
\hline $\mathrm{C}(20)-\mathrm{C}(19)-\mathrm{H}(19)$ & 119.9 \\
\hline $\mathrm{C}(19)-\mathrm{C}(20)-\mathrm{H}(20)$ & 119.9 \\
\hline$C(19)-C(20)-C(21)$ & $120.3(3)$ \\
\hline $\mathrm{C}(21)-\mathrm{C}(20)-\mathrm{H}(20)$ & 119.9 \\
\hline$C(16)-C(21)-C(20)$ & $120.1(3)$ \\
\hline $\mathrm{C}(16)-\mathrm{C}(21)-\mathrm{H}(21)$ & 120.0 \\
\hline $\mathrm{C}(20)-\mathrm{C}(21)-\mathrm{H}(21)$ & 120.0 \\
\hline
\end{tabular}

Symmetry transformations used to generate equiv.alent atoms:

Table 4. Anisotropic displacement parameters $\left(\AA^{2} \times 10^{3}\right)$ for $\mathrm{m} J 18160 \_0 \mathrm{~m} . \quad$ The anisotropic displacement factor exponent takes the form: $\quad-2 \pi^{2}\left[\mathrm{~h}^{2} \mathrm{a}^{* 2} \mathrm{U}^{11}+\ldots+2 \mathrm{~h} \mathrm{k} \mathrm{a} \mathrm{b}^{*} \mathrm{U}^{12}\right]$

$\begin{array}{llllll}\mathrm{U}^{11} & \mathrm{U}^{22} & \mathrm{U}^{33} & \mathrm{U}^{23} & \mathrm{U}^{13} & \mathrm{U}^{12}\end{array}$




\begin{tabular}{|c|c|c|c|c|c|c|}
\hline $\operatorname{Br}(1)$ & $46(1)$ & $77(1)$ & $53(1)$ & $10(1)$ & $33(1)$ & 2(1) \\
\hline $\mathrm{P}(1)$ & $20(1)$ & 12(1) & $21(1)$ & $0(1)$ & $6(1)$ & 1(1) \\
\hline $\mathrm{O}(1)$ & $29(1)$ & $14(1)$ & $29(1)$ & $4(1)$ & $10(1)$ & $6(1)$ \\
\hline $\mathrm{N}(1)$ & $44(2)$ & $46(2)$ & $30(1)$ & $-1(1)$ & $7(1)$ & $-22(2)$ \\
\hline $\mathrm{C}(1)$ & $21(1)$ & $20(2)$ & $22(1)$ & 2(1) & $4(1)$ & $-1(1)$ \\
\hline$C(2)$ & $23(1)$ & $17(1)$ & $21(1)$ & $-1(1)$ & $7(1)$ & $-1(1)$ \\
\hline $\mathrm{C}(3)$ & $21(1)$ & $19(1)$ & $17(1)$ & $1(1)$ & $2(1)$ & $-3(1)$ \\
\hline $\mathrm{C}(4)$ & $29(2)$ & $19(1)$ & $21(1)$ & $0(1)$ & $6(1)$ & $-2(1)$ \\
\hline $\mathrm{C}(5)$ & $31(2)$ & $26(2)$ & $26(1)$ & $-2(1)$ & $9(1)$ & $4(1)$ \\
\hline $\mathrm{C}(6)$ & $30(1)$ & $36(2)$ & $24(1)$ & $-3(2)$ & $11(1)$ & $-5(2)$ \\
\hline $\mathrm{C}(7)$ & $34(2)$ & $28(2)$ & $23(1)$ & $3(1)$ & $7(1)$ & $-9(2)$ \\
\hline $\mathrm{C}(8)$ & $26(1)$ & 19(1) & $20(1)$ & $1(1)$ & 1(1) & $-1(1)$ \\
\hline $\mathrm{C}(9)$ & $27(2)$ & $33(2)$ & $22(1)$ & $0(1)$ & $5(1)$ & $-6(1)$ \\
\hline$C(10)$ & $21(1)$ & $20(1)$ & $24(1)$ & $-5(1)$ & $7(1)$ & $-3(1)$ \\
\hline $\mathrm{C}(11)$ & $44(2)$ & $31(2)$ & $32(2)$ & $-3(1)$ & $16(1)$ & $8(2)$ \\
\hline $\mathrm{C}(12)$ & $55(2)$ & $42(2)$ & $37(2)$ & $-10(2)$ & $25(2)$ & $4(2)$ \\
\hline $\mathrm{C}(13)$ & $33(2)$ & $53(2)$ & $23(1)$ & $-9(2)$ & $13(1)$ & $-9(2)$ \\
\hline $\mathrm{C}(14)$ & $45(2)$ & $50(2)$ & $26(1)$ & $9(2)$ & $13(1)$ & $9(2)$ \\
\hline $\mathrm{C}(15)$ & $47(2)$ & $31(2)$ & $28(1)$ & $2(2)$ & $15(1)$ & $9(2)$ \\
\hline$C(16)$ & 19(1) & $19(1)$ & $18(1)$ & $-4(1)$ & $3(1)$ & $0(1)$ \\
\hline $\mathrm{C}(17)$ & $26(1)$ & $18(1)$ & $24(1)$ & $0(1)$ & $6(1)$ & 1(1) \\
\hline $\mathrm{C}(18)$ & $25(1)$ & $25(2)$ & $29(2)$ & $-4(1)$ & $2(1)$ & $6(1)$ \\
\hline $\mathrm{C}(19)$ & $20(1)$ & $35(2)$ & $34(2)$ & $-5(2)$ & $7(1)$ & 2(1) \\
\hline $\mathrm{C}(20)$ & $25(1)$ & $36(2)$ & $33(2)$ & $-2(2)$ & $12(1)$ & $-6(2)$ \\
\hline $\mathrm{C}(21)$ & $24(1)$ & $19(2)$ & $25(1)$ & $-1(1)$ & $6(1)$ & $-1(1)$ \\
\hline
\end{tabular}


Table 5. Hydrogen coordinates $\left(\mathrm{x} 10^{4}\right)$ and isotropic displacement parameters $\left(\AA^{2} \mathrm{x} 10^{3}\right)$ for mJ18160_0m.

$\mathrm{X}$

$\mathrm{y}$

Z

$\mathrm{U}(\mathrm{eq})$

\begin{tabular}{|c|c|c|c|c|}
\hline $\mathrm{H}(1)$ & 4911 & 6078 & 1858 & 26 \\
\hline $\mathrm{H}(2 \mathrm{~A})$ & 5561 & 2808 & 3419 & 24 \\
\hline $\mathrm{H}(2 \mathrm{~B})$ & 5069 & 4436 & 3538 & 24 \\
\hline $\mathrm{H}(4)$ & 5879 & 1354 & 1960 & 28 \\
\hline $\mathrm{H}(5)$ & 6616 & 1495 & 1261 & 34 \\
\hline $\mathrm{H}(7)$ & 6116 & 7946 & 121 & 35 \\
\hline $\mathrm{H}(8)$ & 5393 & 7835 & 866 & 28 \\
\hline $\mathrm{H}(11)$ & 5581 & 9766 & 5076 & 42 \\
\hline $\mathrm{H}(12)$ & 5657 & 10079 & 6833 & 51 \\
\hline $\mathrm{H}(13)$ & 6105 & 7195 & 8012 & 43 \\
\hline $\mathrm{H}(14)$ & 6435 & 3872 & 7428 & 48 \\
\hline $\mathrm{H}(15)$ & 6355 & 3517 & 5665 & 41 \\
\hline $\mathrm{H}(17)$ & 6627 & 2454 & 4335 & 28 \\
\hline $\mathrm{H}(18)$ & 7510 & 1584 & 4194 & 34 \\
\hline $\mathrm{H}(19)$ & 7956 & 4272 & 3432 & 36 \\
\hline $\mathrm{H}(20)$ & 7530 & 7819 & 2835 & 37 \\
\hline $\mathrm{H}(21)$ & 6663 & 8773 & 3033 & 28 \\
\hline
\end{tabular}

